\title{
MEMS IMU Navigation with Model Based Dead-Reckoning and One-Way-Travel-Time Acoustic Range Measurements for Autonomous Underwater Vehicles
}

\author{
by \\ Lieutenant Commander James H. Kepper, IV, United States Navy \\ B.S., United States Naval Academy, 2005 \\ M.E.M, Old Dominion University, 2012 \\ Submitted to the Joint Program in Applied Ocean Science \& Engineering \\ in partial fulfillment of the requirements for the degree of \\ Master of Science in Oceanographic Engineering \\ at the \\ MASSACHUSETTS INSTITUTE OF TECHNOLOGY \\ and the \\ WOODS HOLE OCEANOGRAPHIC INSTITUTION \\ September 2017 \\ (C)2017 James H. Kepper, IV. \\ All rights reserved. \\ The author hereby grants to MIT and WHOI permission to reproduce and to \\ distribute publicly paper and electronic copies of this thesis document in whole or in \\ part in any medium now known or hereafter created.
}

Author

Joint Program in Applied Ocean Science \& Engineering Massachusetts Institute of Technology \& Woods Hole Oceanographic Institution

August 3, 2017

Certified by .

James C. Kinsey

Associate Scientist

Woods Hole Oceanographic Institution

Thesis Supervisor

Accepted by

Rohan Abeyaratne

Chairman, Committee on Graduate Students Massachusetts Institute of Technology

Accepted by

Henrik Schmidt

Chairman, Joint Committee for Applied Ocean Science \& Engineering Massachusetts Institute of Technology Woods Hole Oceanographic Institution 


\title{
MEMS IMU Navigation with Model Based Dead-Reckoning and One-Way-Travel-Time Acoustic Range Measurements for Autonomous Underwater Vehicles
}

\author{
by
}

\author{
Lieutenant Commander James H. Kepper, IV, United States Navy \\ Submitted to the Joint Program in Applied Ocean Science \& Engineering \\ Massachusetts Institute of Technology \\ \& Woods Hole Oceanographic Institution \\ on August 3, 2017, in partial fulfillment of the \\ requirements for the degree of \\ Master of Science in Oceanographic Engineering
}

\begin{abstract}
Recent advances in acoustic navigation methodologies are enabling the way for AUVs to extend their submerged mission time and maintain a bounded XY position error. Additionally, advances in inertial sensor technology have drastically lowered the size, power consumption, and cost of these sensors. Nonetheless, these sensors are still noisy and accrue error over time. This thesis builds on the research and recent developments in single beacon one-waytravel-time (OWTT) acoustic navigation and investigates the degree of bounding position error for small AUVs with a minimal navigation strap-down sensor suite, relying mostly on a consumer grade microelectromechanical system (MEMS) inertial measurement unit (IMU) and a vehicle's dynamic model velocity. An implementation of an Extended Kalman Filter (EKF) that includes IMU bias estimation and coupled with a range filter, is obtained in the field on two OceanServer Technology, Inc. Iver2 AUVs and one Bluefin Robotics SandShark $\mu \mathrm{AUV}$. Results from these field trials on Ashumet Pond of Falmouth, Massachusetts, the Charles River of Cambridge, Massachusetts, and Monterey Bay near Santa Cruz, California show a navigation solution accuracy comparable to current standard navigation techniques.

Thesis Supervisor: James C. Kinsey

Title: Associate Scientist

Woods Hole Oceanographic Institution
\end{abstract}




\section{Acknowledgments}

This research was funded by the U.S. Navy's Civilian Institution Program with the MIT/WHOI Joint Program. The Keck Institute for Space Studies "Satellites to Seafloor" research project and the Woods Hole Oceanographic Institution provided resources for equipment, travel, and professional development activities.

I am incredibly grateful to the U.S. Navy's Submarine Force for providing this opportunity to study at these two world class institutions and work closely with very talented people.

Thank you to Dr. James Kinsey, my advisor, for his mentorship and guidance throughout my research. Amidst his very busy schedule and transition with an additional job, he always found time for me and providing feedback on my work. I wish him the best of luck with continued success at Humatics and as you start your new family.

Thank you to Dr. Brian Claus, my office-mate and friend. Throughout the year, his ingenuity, insight, and professional expertise helped me to develop my skills and to expand my knowledge in the area of underwater robotics. He always helped me tackle those petty frustrations that we all encounter in this field. Without Brian, none of these experiments could have been accomplished. I wish him and his family the very best as he begins his new job.

Thank you to Professors Henrik Schmidt and Arthur Baggeroer for their guidance and support of the Navy students. Thank you to Dr. Mike Benjamin for his support and instruction with helping me in developing my skills. Thank you to Dr. Hanu Singh and Dr. Sarah Webster for your feedback on my ideas throughout my research.

Thank you to the Joint Program staff, all those in the Academic Programs Office, and Mrs. Leslie Regan at MIT for answering any and all questions about the program requirements and providing oversight to ensure that I successfully completed the program.

Thank you to all those in the Deep Submergence Lab, especially those in the Blake Building. The weekly coffee hours and daily conversations in the P-Ways helped to make my experience here one of my best tours. Thanks to Dr. Rich Camilli for providing mentorship and feedback on my work as well as providing many of his materials for the experiments. Thank you especially to Ms. Judy Fenwick for processing all of the necessary paperwork and work requests - little things that help our experience be that much better! And, thank 
you, Judy, for your gifts for my two little girls throughout the year.

Thank you to all of my JP classmates, especially to Jeffrey Mei and Eeshan Bhatt. Thanks for all of your help on problem sets, computer programing, and making time to answer my questions. Thanks to Nick Rypkema and his assistance with the SandShark data.

Thank you to my wonderful wife, Ashley, and my daughters, Monica and Maria, for all of your love and support throughout these past two years. We have had great experiences with our time in New England, and I am forever grateful to have you three next to me as we continue on our journey together.

Lastly, I thank God for His grace and His guidance in bringing my family here. We are thankful for all of the fruits and blessings that have come from our tour in New England. 


\section{Contents}

1 Introduction $\quad 13$

1.1 Motivation . . . . . . . . . . . . . . . . 13

1.2 AUV Navigation - A Short Literature Review . . . . . . . . . . . . . . . 14

1.2.1 Acoustic Navigation . . . . . . . . . . . . . . . . . 14

1.2.2 Navigation with Inertial Measurement Units (IMU) . . . . . . . . . 16

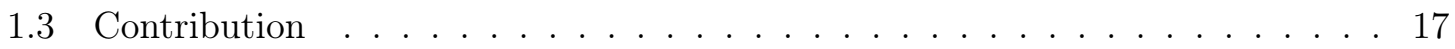

2 Navigation Model $\quad 19$

2.1 Kalman Filter - A Brief Overview . . . . . . . . . . . . . . . . . . . 19

2.2 IMU EKF Model for AUVs . . . . . . . . . . . . . . . . . . . . . . 21

$2.2 .1 \quad$ Plant Model . . . . . . . . . . . . . . . . . . . . . . . 21

2.2 .2 Observation Models _. . . . . . . . . . . . . . . . . 26

2.2 .3 Coupled Range Filter . . . . . . . . . . . . . . . . . . . . . 30

2.3 Attitude Complementary Filter . . . . . . . . . . . . . . . . . 32

3 Experiment Configuration $\quad 35$

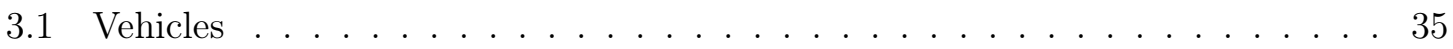

3.1 .1 OceanServer Technology, Inc. Iver2 AUV . . . . . . . . . . . . . . 35

3.1.2 Bluefin Robotics SandShark AUV . . . . . . . . . . . . . . . 36

3.2 Acoustic Communications . . . . . . . . . . . . . . . . 38

3.2 .1 Iver2 AUVs Communications . . . . . . . . . . . . . . . 38

3.2.2 SandShark $\mu$ AUV Acoustic Communications . . . . . . . . . . . . 39

3.3 IMU EKF Implementation . . . . . . . . . . . . . . . . . . . . . . . . 39 
4.1 Ashumet Pond Trials - October 2016 . . . . . . . . . . . . . . . . . . . 42

4.1 .1 Trajectory Summary . . . . . . . . . . . . . . . . . . . . . 42

4.1 .2 Performance Analysis . . . . . . . . . . . . . . . . . . 43

4.2 Charles River Trials - October $2016 \ldots \ldots \ldots \ldots$

4.2 .1 Trajectory Summary . . . . . . . . . . . . . . . . 52

4.2 .2 Performance Analysis . . . . . . . . . . . . . . . . . . . . . 52

4.3 Ashumet Pond Trials - April $2017 \ldots \ldots$. . . . . . . . . . 55

4.3 .1 Trajectory Summary . . . . . . . . . . . . . 56

4.3.2 CL EKF Range Sensitivity Discussion . . . . . . . . . . . . . . 57

4.3 .3 Performance Analysis . . . . . . . . . . . . . . . . 60

4.4 Discussion . . . . . . . . . . . . . . . . . . . . . . . 69

4.4.1 IMU Linear Acceleration Analysis . . . . . . . . . . . . . . . . . 71

4.4 .2 Singularity Analysis . . . . . . . . . . . . . . . . . . 71

4.4 .3 Performance Summary . . . . . . . . . . . . . . . . . 72

4.5 Monterey Bay Trials - September $2016 \ldots \ldots$. . . . . . . . . . 75

4.5 .1 Trajectory Summary . . . . . . . . . . . . . 76

4.5.2 Performance Analysis _ . . . . . . . . . . . . . . . . . . 77

$\begin{array}{llr}5 & \text { Conclusion } & 79\end{array}$

5.1 Research Summary . . . . . . . . . . . . . . . . . . . . . . 79

5.2 Recommendations for Future Research . . . . . . . . . . . . . . 80

5.3 Future Applications $\ldots \ldots \ldots \ldots$. . . . . . . . . . . . . 81 


\section{List of Figures}

2-1 Block Diagram for the IMU EKF with a Coupled Range Filter . . . . . . . . 22

3-1 OceanServer Technology, Inc. Iver2 AUVs onboard the R/V Shana Rae. . . . 36

3-2 Iver2 AUV Systems Configuration Layout . . . . . . . . . . . . . . . . 36

3-3 Bluefin Robotics SandShark $\mu \mathrm{AUV} \ldots \ldots$. . . . . . . . . . . . 37

3-4 Acoustic Communications System Block Diagram . . . . . . . . . . . . . 38

4-1 October 2016 Field Trials Iver2 AUV Position Summary . . . . . . . . . . . . 44

4-2 October 2016 Field Trials Iver-106 EKF Performance Summary . . . . . . . . 45

4-3 October 2016 Field Trials Iver-136 EKF Performance Summary . . . . . . . . 46

4-4 October 2016 Iver-106 XY Trajectory Summary with DVL Measurements . 47

4-5 October 2016 Iver-106 IMU EKF (DVL-Aided) Error Summary . . . . . . . . 48

4-6 October 2016 Iver2 IMU EKF Bias Estimation Summary . . . . . . . . . . . . 50

$4-7$ SandShark Raw OWTT Ranges . . . . . . . . . . . . . . . . . . . . 52

4-8 SandShark XY Trajectory without the Coupled Range Filter . . . . . . . . . 53

4-9 SandShark Processed OWTT Ranges . . . . . . . . . . . . . . . . 54

4-10 SandShark AUV EKF XY Position Summary . . . . . . . . . . . . . . . 54

4-11 SandShark AUV Error Summary . . . . . . . . . . . . . . . 55

4-12 April 2017 Field Trials Iver2 Planned Mission Trajectories . . . . . . . . . . 56

4-13 April 2017 Field Trials Iver2 Closed-loop EKF XY Trajectory Summary . . . 58

4-14 April 2017 Field Trials Iver2 Raw OWTT Range Time Series Summary . . . . 59

4-15 April 2017 Field Trials Iver-106 XY Trajectory Plots . . . . . . . . . . . . . 62

4-16 April 2017 Field Trials Iver-106 IMU EKF Error . . . . . . . . . . . . . . . 63

4-17 April 2017 Field Trials Iver-106 EKF Error Summary . . . . . . . . . . . . . 64

4-18 April 2017 Field Trials Iver-136 IMU EKF Trajectory Plot . . . . . . . . . . . 65 
4-19 April 2017 Field Trials Iver-136 IMU EKF Error Summary . . . . . . . . . . . 66

4-20 April 2017 Field Trials Iver-136 EKF Error Summary . . . . . . . . . . . . . . 67

4-21 April 2017 Field Trials Raw \& Processed OWTT Range Measurement Summary 69

4-22 April 2017 Field Trials Iver-106 \& Iver-136 XY Position Time Series Summary 70

4-23 April 2017 Iver-136 Singularity Analysis . . . . . . . . . . . . . . . . . . . 73

4-24 2016 \& 2017 Field Trial Performance Summary Bar Charts . . . . . . . . . . 74

$4-25$ Monterey Bay, CA . . . . . . . . . . . . . . . . . . 75

4-26 Monterey Bay, CA Iver-136 Trajectory Summary . . . . . . . . . . . . 76

4-27 Monterey Bay, CA Iver-136 Time Series Error Summary . . . . . . . . . . . . 77 


\section{List of Tables}

3.1 Pololu \& Microstrain IMU Statistics . . . . . . . . . . . . . . . 37

4.1 Navigation Solution Description Summary . . . . . . . . . . . . . . . 42

4.2 October 2016 Field Trials Iver2 IMU EKF Parameter Values . . . . . . . . . . 43

4.3 October 2016 Field Trials Iver-106 EKF Average Error Summary . . . . . . . 45

4.4 October 2016 Field Trials Iver-106 DVL-aided IMU EKF Parameter Values 46

4.5 October 2016 Field Trials Iver-106 EKF Performance Summary . . . . . . . . 47

4.6 October 2016 Field Trials Iver2 IMU EKF Beacon \& Bias Analysis Summary 48

4.7 October 2016 Field Trials IMU EKF Performance with Bias Estimation . . . 51

4.8 SandShark IMU EKF Parameter Values . . . . . . . . . . . . . . . . . . . 51

4.9 SandShark Navigation Performance Summary . . . . . . . . . . . . . 55

4.10 April 2017 Iver-106 IMU EKF Parameter Values . . . . . . . . . . . . . . 61

4.11 April 2017 Field Trials Iver-106 EKF Performance Summary . . . . . . . . . . 62

4.12 April 2017 Field Trials Iver-136 IMU EKF Parameter Values . . . . . . . . . . 64

4.13 April 2017 Field Trials Iver-136 EKF Performance Summary . . . . . . . . . . 67

4.14 April 2017 Field Trials Coupled Range Filter Performance Summary . . . . . 69

4.15 IMU EKF Numerical Singularity Analysis Summary . . . . . . . . . . . . 72 
THIS PAGE INTENTIONALLY LEFT BLANK 


\section{Chapter 1}

\section{Introduction}

\subsection{Motivation}

Progress in underwater robotic systems brings both the scientific research and military communities a reality that is reachable in deploying multiple autonomous underwater vehicles (AUVs) throughout the entirety of the water column. With this capability, the scientific community's surveys can extend both their spatial and temporal scales, leading to further investigation and understanding of biochemical exchange between the ocean and the atmosphere coming from hydrothermal vent activity[43]. Likewise, the military defense community can both extend mission coverage and minimize time on station for a variety of intelligence missions and mine clearance operations. Additionally, a navigation methodology that yields accurate navigation and localization within a certain threshold will benefit both communities. Although these methods exist, however, higher accuracy is traditionally at the expense for higher costs platforms. This trade-off limits the ability for these communities to deploy multiple vehicles on a single mission and limits the operational areas in the water column. In order for these communities to expand their operations with multiple robotic platforms (either homogeneous or heterogeneous), a means of inexpensive, yet precise and accurate, navigation will help enable missions throughout the water column. This thesis addresses this problem by researching a navigation solution based on a microelectromechanical systems (MEMS) inertial measurement unit (IMU), a vehicle's dynamic model velocity, and acoustic positioning. 


\subsection{AUV Navigation - A Short Literature Review}

\subsubsection{Acoustic Navigation}

Global Positioning Systems (GPS) provide land, air, and sea surface robots with highly accurate, absolute position measurements, thus enabling highly accurate navigation solutions. However, for robots operating underwater, the lack of GPS signals pose unique challenges. AUVs typically navigate by means of various sensors to compute a dead-reckoned odometry $[33,38]$. However, without external aiding, position error grows unbounded over time.

AUVs can surface periodically to obtain GPS measurements, thus updating position estimates and reducing its error. However, periodic surfacing limits the energy consumption efficiency (thus reducing its overall mission time) and prevents covert missions needed for military applications and, for deep AUVs, periodic surfacing requires they leave the areas of interest. One such solution for providing absolute position measurements while the vehicle remains submerged is acoustic time of flight (TOF) navigation. Long Baseline (LBL) navigation [29], in which an AUV triangulates its position to fixed surveyed acoustic transponders. LBL systems require time-consuming transponder surveys, require expensive ship time, and limit the mission coverage area. Ultra-Short Baseline (USBL) navigation [39] is an alternative method that does not require fixed transponders on the ocean bottom, but it still requires the ship, with its expensive operational time, to remain on station and not conduct other simultaneous missions in different areas. Additionally, USBL systems require the AUVs to transmit acoustic packets to the ship's array, making this scheme less desirable due to the energy consumption costs. Also, for USBL systems equipped with an acoustic modem, the vehicle can receive position updates from the topside beacon, but these updates are time delayed.

Advances in single beacon acoustic navigation over the past decade present an alternative means for bounding XY navigation error since depth is easily bounded by pressure depth sensors. In this method, a surface beacon, which has access to GPS, transmits its position to a submerged vehicle in an acoustic packet. Highly accurate and synchronized clocks (a chip-scale atomic clock or a temperature compensated crystal oscillator coupled with a pulseper-second clock) $[25,26]$ on both the beacon and the receiving vehicle accurately measure the acoustic packet's one-way-travel-time (OWTT) TOF. From this TOF calculation, a range between the surface beacon and the vehicle is determined using the water column's sound 
speed, and this range is used to constrain the AUV's position estimate, thus bounding its XY position error. Many successful experimental results using single beacon OWTT range measurements over the past decade prove this capability of bounding position error $[16,17,18,51,53,56,54]$. More recently, a moving short baseline navigation solution that incorporated two transducers on the surface beacon made use of OWTT range constraints [57]. For this method, when the AUVs are distant from the surface ship, the two surface beacons act as a single beacon providing OWTT range measurements. However, when the AUVs are near the surface beacon, navigation and localization improve due to the two transponders. These results incorporate a variety of state estimation algorithms, primarily consisting of position displacement odometry determined by a Doppler Velocity Log (DVL), which is constrained by the OWTT range measurements. The major advantage for single beacon OWTT navigation, in addition to submerged XY position constraints, is the ability to deploy multiple vehicles that each simultaneously receive position data acoustic packets from the same surface beacon, and hence, the position update rate for each vehicle remains constant.

Observability is important with range only measurement methods. Since a range measurement only provides a constraint and not an absolute position measurement, only certain AUV trajectories using inertial measurement sensors are observable. Previous work proved that a system is observable by analysis of the Fisher Information Matrix [42]. Others proved trajectories are observable except those that are straight line segments passing through the origin (i.e. the beacon) $[22,23]$. Other developments include a belief space planning algorithm to optimize trajectories of a team of AUVs in order to enhance observability and thus make the navigation solutions from these inter-vehicle ranges more accurate [49].

With the success of using OWTT range measurements from a single beacon to constrain XY position error, multiple vehicle navigation algorithms are using OWTT inter-vehicle range measurements to further constrain error. The main challenge in using inter-vehicle ranges for position estimates is overconfidence in the solution. Prior work in this area includes development of an algorithm in which the broadcasting vehicles share their pose and covariance estimates. This information is then used in a filter on a receiving vehicle to ensure conservative covariance estimates by preventing the use of measurements from the same origin more than once [3]. Another approach is an algorithm that computes odometry factors from the transmitting vehicle to prevent overconfidence in the receiving vehicle's 
solution based on inter-vehicle range measurements [50].

\subsubsection{Navigation with Inertial Measurement Units (IMU)}

IMUs most commonly contain three orthogonal accelerometers, gyroscopes, and magnetometers that respectively measure linear acceleration, angular rates, and magnetic field strength. Thus, IMUs serve as a navigation sensor to determine a vehicle's attitude as well as serve as an odometry input by integrating the linear accelerations to obtain velocity and position. While reductions in size, power consumption, and cost of MEMS IMUs are occurring, their higher sensor noise levels and inaccuracy make them insufficient for many navigation applications compared to other high-end inertial navigation systems (INS). For example, compared to a commercial grade INS (that costs on the order of tens of thousands of dollars, power consumption on the order of tens of watts, exhibits a drift of $0.001-1^{\mathrm{o}} / \mathrm{hr}$ for a fiber optic gyroscope, and 0.001 - $1 \mathrm{mg}$ of acceleration bias for a pendulus accelerometer), a MEMS IMU gyroscope drifts greater than $60^{\circ} / \mathrm{hr}$, and its accelerometer exhibits $0.01-1$ mg of bias [12], but it only costs on the order of tens of dollars and consumes power on the order of tens of milliwatts.

Many methods and algorithms reduce the errors of MEMS IMUs to make them suitable for underwater vehicle navigation. One such method is assessing an IMU's error statistics and then determining how best to select an IMU for a particular application [13]. Other methods consist of an Extended Kalman Filter (EKF) to estimate attitude in a quarternion representation with depth measurements for improving accuracy [34]. Further, double integration of IMU linear accelerations provide a measurement for odometry in a navigation solution. A fused Kalman Filter consisting of GPS as well as IMU linear accelerations showed acceptable accuracy of a remotely operated vehicle but only consisted of a trial run of ten seconds [35]. Better results of IMU accelerations as an odometry input are provided by an on-line EKF that fused IMU sensor measurements and GPS speed-over-ground measurements on a terrain vehicle that resulted in a root-mean-square (RMS) error of 17.4 meters over a distance traveled of approximately 8.75 kilometers in 51 minutes [41]. Additionally, in simulation, a combined translational and attitude observer, that used an IMU for both attitude and odometry, fused with DVL speed measurements, resulted in a RMS position error of 0.5 meters over 250 seconds of mission time [14].

Due to higher noise levels, bias errors, and drift errors from the accelerometers and gy- 
roscopes, the double integration of the linear accelerations can lead to quite large position errors over time [45]. Since bias errors are known to drift over time, continuously updating and subtracting this bias error from a measurement can improve performance. Three angular-rate aided estimators [46] improved bias estimation over previous methods of the TWOSTEP [2] and the attitude-independent EKF [10]. Others have improved performance by using an EKF to estimate bias and scale factor errors for both accelerometers and gyroscopes after a calibration procedure [5]. Lastly, a first order Gauss-Markov process, used to model bias estimation in a Kalman Filter, showed convergence within 60 seconds for the gyroscopes and within 1 second for the accelerometers [47].

\subsection{Contribution}

This thesis builds upon the research in single beacon OWTT acoustic navigation and the use of MEMS IMUs for measurements to determine AUV odometry and attitude. This research contributes the following:

(1) A navigation solution (referred to herein as the IMU EKF) determined by an EKF that incorporates a MEMS IMU, a vehicle's dynamic model velocity (based on propeller turn count) for speed measurements, and acoustic TOF range measurements and is comparable to current standard navigation solutions.

(2) Accelerometer bias estimation in the IMU EKF to reduce the inherent MEMS IMU accelerometer error.

(3) A coupled range filter to the IMU EKF in order to preclude processing faulting acoustic range measurements from hardware defects or environmental variability.

Field data from three different controlled-environment experiments in local waters and one open ocean experiment are presented proving this concept with a comparison of different navigation solutions. Items 1 and 2 along with results from the October 2016 field experiments are presented in a prior work [32].

The remainder of this thesis is organized as follows: Chapter 2 describes the EKF model used in this navigation solution. Chapter 3 describes the platforms used and the field experiment configurations conducted in Ashumet Pond of Falmouth, MA, the Charles River of Cambridge, MA, and Monterey Bay, CA. Chapter 4 discusses the field results, and Chapter 5 closes with conclusions and recommendations for future work. 
THIS PAGE INTENTIONALLY LEFT BLANK 


\section{Chapter 2}

\section{Navigation Model}

\subsection{Kalman Filter - A Brief Overview}

The Kalman Filter [31] is a benchmark state estimator in current navigation solution methods. Its wide use and applicability in this field are the reasons for choosing the Extended Kalman Filter (EKF) [37], a nonlinear variant of the Kalman Filter, to serve as the state estimator for this thesis.

The Kalman Filter estimates a state by propagating a linear dynamic system's state perturbed by white noise and infers updates to the state by related measurements also perturbed by white noise as seen in the equations below:

$$
\begin{gathered}
\dot{\mathbf{x}}(t)=\mathbf{A} \mathbf{x}(t)+\mathbf{D} \mathbf{w}(t) \\
\mathbf{z}(t)=\mathbf{H} \mathbf{x}(t)+\mathbf{v}(t)
\end{gathered}
$$

In Equations (2.1 - 2.2), $\mathbf{x}(\mathrm{t})$ is the system's state vector, $\mathbf{A}$ is the system transition matrix, $\mathbf{D}$ is the noise coefficient matrix, $\mathbf{w}(\mathrm{t})$ is the system's white dynamic process noise, $\mathbf{z}(\mathrm{t})$ is the measurement, $\mathbf{H}$ is the measurement-to-state mapping matrix, and $\mathbf{v}(\mathrm{t})$ is the measurement white noise. The plant process noise, $\mathbf{w}(\mathrm{t})$, and measurement noise, $\mathbf{v}(\mathrm{t})$, are considered zero-mean, Gaussian white noise, such that,

$$
\mathbf{w}(t) \sim \mathcal{N}(0, \mathbf{Q}), \mathbf{v}(t) \sim \mathcal{N}(O, \mathbf{R})
$$

where $\mathbf{Q}$ is defined as the system process-noise variance matrix, and $\mathbf{R}$ is the measurement 
noise variance matrix.

Performing a discretization of the continuous time system expressed in equations (2.1) and (2.2), the following discrete-time dynamic system is presented:

$$
\mathbf{x}_{\mathrm{k}+1}=\mathbf{F x}_{\mathrm{k}}+\boldsymbol{v}_{k}
$$

The discrete time transition matrix, $\mathbf{F}$, and discrete-time process noise, $\boldsymbol{v}$, are defined as follows for time step, $d t$ : [4]

$$
\begin{gathered}
\mathbf{F}=e^{\mathbf{A} d t} \\
\boldsymbol{v}(k)=\int_{0}^{d t} e^{\mathbf{A}(d t-\tau)} \mathbf{D} \mathbf{w}(k d t+\tau) d \tau
\end{gathered}
$$

During each time step in the Kalman Filter process, a prediction step is processed that propagates the system model followed by a measurement update step. This update step incorporates a measurement, which is related to the system's state, and updates the predicted state value with a gain factor (weighted by system statistics) and the measurement innovation (i.e., the difference between the actual and the predicted measurements). The discrete-time Kalman Filter prediction step equations are as follows:

$$
\begin{gathered}
\hat{\mathbf{x}}_{k+1}^{-}=\mathbf{F} \hat{\mathbf{x}}_{k}^{+}+\mathbf{B} \mathbf{u}_{\mathrm{k}} \\
\mathbf{P}_{k+1}^{-}=\mathbf{F} \mathbf{P}_{k}^{+} \mathbf{F}^{T}+\mathbf{Q}
\end{gathered}
$$

where $\mathbf{u}$ is a discrete-time control input function, $\mathbf{B}$ is the coefficient matrix, $\mathbf{P}$ is the system's covariance matrix, and $\mathbf{Q}$ is the system's process noise covariance matrix defined as

$$
\mathbf{Q}=E\left[\boldsymbol{v}(k) \boldsymbol{v}(k)^{T}\right]
$$

The measurement update equations are as follows, where $\mathbf{K}$ is the Kalman gain matrix:

$$
\begin{gathered}
\mathbf{K}_{\mathrm{k}}=\mathbf{P}_{k}^{-} \mathbf{H}_{k}^{T}\left(\mathbf{R}_{\mathrm{k}}+\mathbf{H}_{\mathrm{k}} \mathbf{P}_{k}^{-} \mathbf{H}_{k}^{T}\right)^{-1} \\
\hat{\mathbf{x}}_{k}^{+}=\hat{\mathbf{x}}_{k}^{-}+\mathbf{K}_{\mathrm{k}}\left(\mathbf{z}_{\mathrm{k}}-\mathbf{H}_{\mathrm{k}} \hat{\mathbf{x}}_{k}^{-}\right) \\
\mathbf{P}_{k}^{+}=\left(\mathbf{I}-\mathbf{K}_{\mathrm{k}} \mathbf{H}_{\mathrm{k}}\right) \mathbf{P}_{k}^{-}\left(\mathbf{I}-\mathbf{K}_{\mathrm{k}} \mathbf{H}_{\mathrm{k}}\right)^{T}+\mathbf{K}_{\mathrm{k}} \mathbf{R}_{k} \mathbf{K}_{k}^{T}
\end{gathered}
$$


The "Joseph form" of the Riccati equation (2.11) is used to ensure positive definiteness [7].

\subsection{IMU EKF Model for AUVs}

A summary of the entire IMU EKF algorithm used in this research is presented in Algorithm 1. An overview block diagram of this EKF implemented in real time on the AUVs is displayed

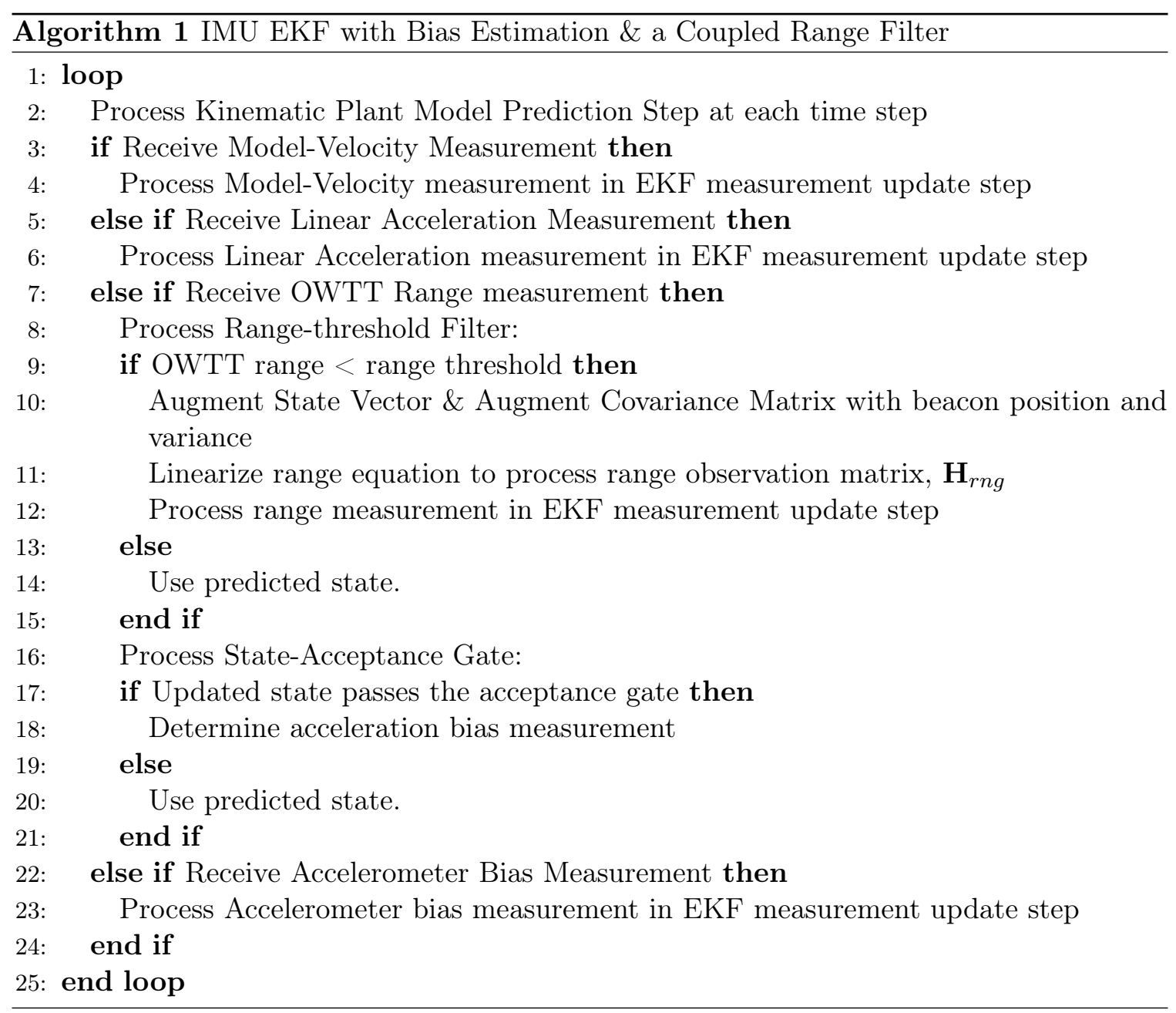

in Figure 2-1. This block diagram portrays all of the different measurements and processes that are incorporated in this navigation model. The details of each block are discussed in the following sections.

\subsubsection{Plant Model}

Since the OWTT range measurements are non-linear, the Kalman Filter variant used is an $\mathrm{EKF}$, in which the nonlinear processes and/or measurements are linearized by a first order 


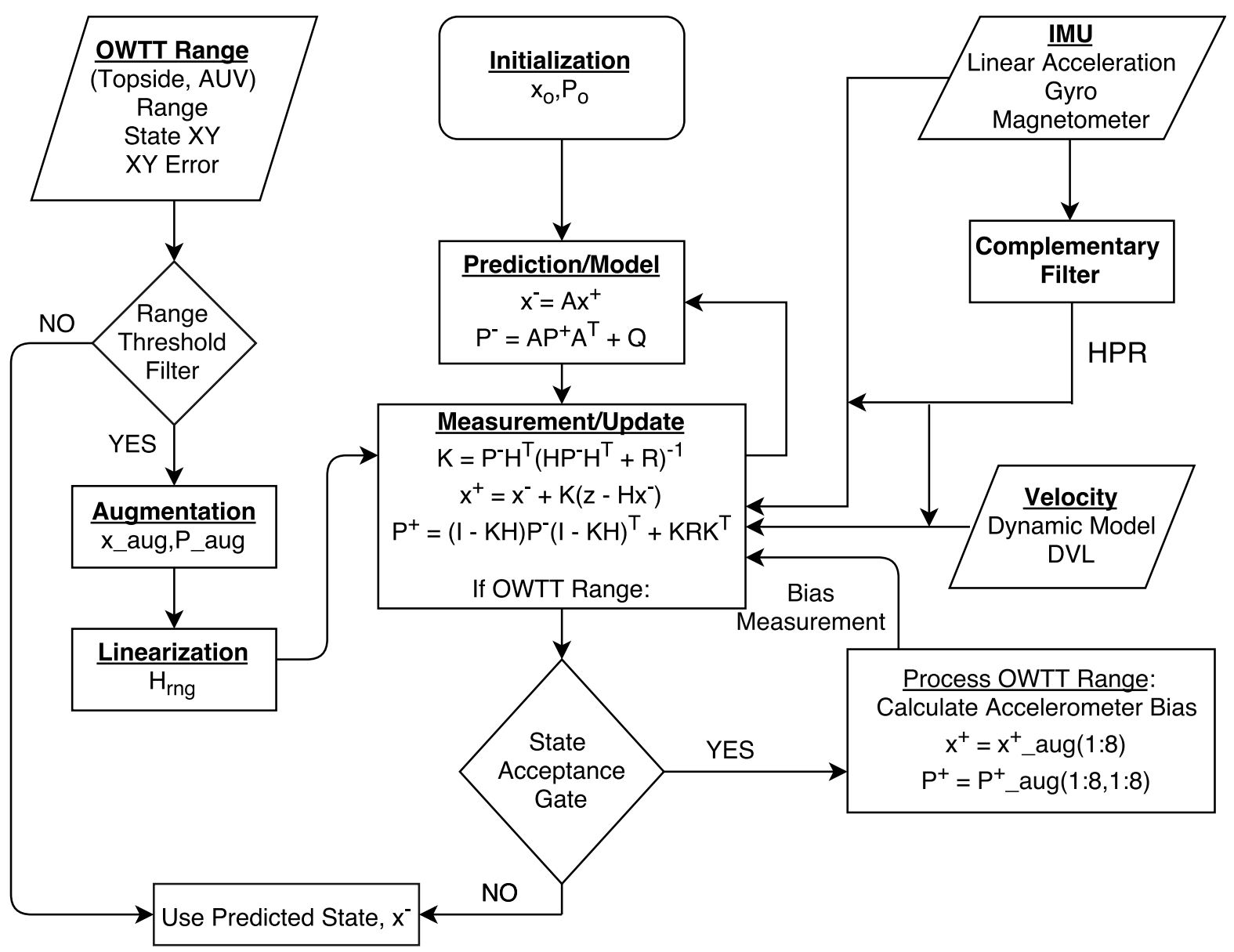

Figure 2-1: Block Diagram for the IMU EKF with a coupled range filter. This block diagram shows the start (rounded box), the processes (square), the input measurements (trapezoid), and the decision points by the coupled range filter (diamond).

Taylor Series Expansion and evaluated at the state's value for that time step. Using the linear Kalman Filter discussion presented in Section 2.1, the IMU EKF model is detailed in this section.

The vehicle's state vector, $\mathbf{x}_{\mathbf{v}}$, is defined as follows:

$$
\mathbf{x}_{\mathbf{v}}=\left[x, y, u, v, a_{x}, a_{y}, b_{x}, b_{y}\right]^{T}
$$

where each $x, y$ pair is the vehicle's XY position in the world frame (i.e., positive $x$ is East, positive $y$ is North, and positive $z$ is down), each $u, v$ pair is the vehicle's XY velocities in the world frame, and each $a_{x}, a_{y}$ pair is the vehicle's XY linear accelerations in the world frame. The $b_{x}, b_{y}$ elements are the respective XY acceleration bias terms in the world frame. Since attitude and depth are adequately instrumented, the $z$ dimension parameters can be easily 
measured and estimated. Thus, the three-dimensional OWTT range is projected into the horizontal plane, and only the XY position, velocity, acceleration, and biases are estimated.

The system model used for this EKF is a Continuous Wiener Process Constant Acceleration model [4] combined with an accelerometer bias model. The constant acceleration model is used for several reasons. This model allows use of the IMU linear accelerations as measurement in the EKF. Also, this kinematic model is applicable to any type of AUV since it does not incorporate vehicle dynamics, such as drag, thrust, or steering models. Additionally, since many AUV missions consist of long, straight, constant-velocity tracks, linear accelerations are approximately constant at zero. The disadvantage to this model is its inaccuracy during turns. However, this issue is mitigated when the EKF error returns to convergence once the vehicle's motion has returned to a constant straight line path [22]. The system transition matrix, A, and the noise coefficient matrix, D, from Equation (2.1) are defined as follows:

$$
\mathbf{A}=\left[\begin{array}{cccccccc}
0 & 0 & 1 & 0 & 0 & 0 & 0 & 0 \\
0 & 0 & 0 & 1 & 0 & 0 & 0 & 0 \\
0 & 0 & 0 & 0 & 1 & 0 & -1 & 0 \\
0 & 0 & 0 & 0 & 0 & 1 & 0 & -1 \\
0 & 0 & 0 & 0 & 0 & 0 & 0 & 0 \\
0 & 0 & 0 & 0 & 0 & 0 & 0 & 0 \\
0 & 0 & 0 & 0 & 0 & 0 & 0 & 0 \\
0 & 0 & 0 & 0 & 0 & 0 & 0 & 0
\end{array}\right]
$$

$$
\mathbf{D}=\left[\begin{array}{llllllll}
0 & 0 & 0 & 0 & 1 & 1 & 0 & 0
\end{array}\right]^{T}
$$

Conducting a normal discretization of the continuous system using Equation (2.4) with 
sampling period, $d t$, the discrete time transition matrix, $\mathbf{F}$, is determined:

$$
\mathbf{F}=\left[\begin{array}{cccccccc}
1 & 0 & d t & 0 & \frac{1}{2} d t^{2} & 0 & -\frac{1}{2} d t^{2} & 0 \\
0 & 1 & 0 & d t & 0 & \frac{1}{2} d t^{2} & 0 & -\frac{1}{2} d t^{2} \\
0 & 0 & 1 & 0 & d t & 0 & -d t & 0 \\
0 & 0 & 0 & 1 & 0 & d t & 0 & -d t \\
0 & 0 & 0 & 0 & 1 & 0 & 0 & 0 \\
0 & 0 & 0 & 0 & 0 & 1 & 0 & 0 \\
0 & 0 & 0 & 0 & 0 & 0 & 1 & 0 \\
0 & 0 & 0 & 0 & 0 & 0 & 0 & 1
\end{array}\right]
$$

A first-order Gauss-Markov (GM) process is used to model the accelerometer bias as a random process. This model is chosen to model the slowly time varying accelerometer bias because of its bounded uncertainty characteristic [15, 20,47]. In discrete time, the bias, b, is written as

$$
\mathbf{b}_{k}=e^{-\frac{d t}{T_{c}}} \mathbf{b}_{k-1}+\mathbf{w}_{b, k}
$$

where $d t$ is the sampling period, $T_{c}$ is the correlation time constant, and $\mathbf{w}_{b}$ is zero-mean bias model process white noise with variance, $\sigma_{w, b}^{2}$ with a tunable parameter, $\sigma_{b}^{2}$ :

$$
\sigma_{w, b}^{2}=\sigma_{b}^{2}\left(1-e^{-\frac{2 d t}{T_{c}}}\right)
$$

The discrete time GM process model in Equation (2.16) for the bias terms are substituted in the appropriate elements of the discrete system transition matrix, $\mathbf{F}$, resulting in

$$
\mathbf{F}=\left[\begin{array}{cccccccc}
1 & 0 & d t & 0 & \frac{1}{2} d t^{2} & 0 & -\frac{1}{2} d t^{2} & 0 \\
0 & 1 & 0 & d t & 0 & \frac{1}{2} d t^{2} & 0 & -\frac{1}{2} d t^{2} \\
0 & 0 & 1 & 0 & d t & 0 & -d t & 0 \\
0 & 0 & 0 & 1 & 0 & d t & 0 & -d t \\
0 & 0 & 0 & 0 & 1 & 0 & 0 & 0 \\
0 & 0 & 0 & 0 & 0 & 1 & 0 & 0 \\
0 & 0 & 0 & 0 & 0 & 0 & e^{-\frac{d t}{T_{c}}} & 0 \\
0 & 0 & 0 & 0 & 0 & 0 & 0 & e^{-\frac{d t}{T_{c}}}
\end{array}\right]
$$


To derive the discrete time process noise matrix, $\mathbf{Q}$, recall the discrete time process noise from Equation (2.5):

$$
\boldsymbol{v}(k)=\int_{0}^{d t} e^{\mathbf{A}(d t-\tau)} \mathbf{D} \mathbf{w}(k d t+\tau) d \tau
$$

Initially, for the derivation, the transition matrix, $\mathbf{A}$, and the noise coefficient matrix, $\mathbf{D}$, are based on the two-dimensional constant acceleration model only. In other words, these two matrices do not contain the acceleration bias terms. Therefore, for the purpose of using Equation (2.19), the $\mathbf{A}$ and $\mathbf{D}$ matrices are as follows:

$$
\begin{aligned}
\mathbf{A} & =\left[\begin{array}{llllll}
0 & 0 & 1 & 0 & 0 & 0 \\
0 & 0 & 0 & 1 & 0 & 0 \\
0 & 0 & 0 & 0 & 1 & 0 \\
0 & 0 & 0 & 0 & 0 & 1 \\
0 & 0 & 0 & 0 & 0 & 0 \\
0 & 0 & 0 & 0 & 0 & 0
\end{array}\right] \\
\mathbf{D} & =\left[\begin{array}{llllll}
0 & 0 & 0 & 0 & 1 & 1
\end{array}\right]^{T}
\end{aligned}
$$

The discrete time process noise matrix, $\mathbf{Q}$, with gain parameter, $\tilde{q}$, is then derived:

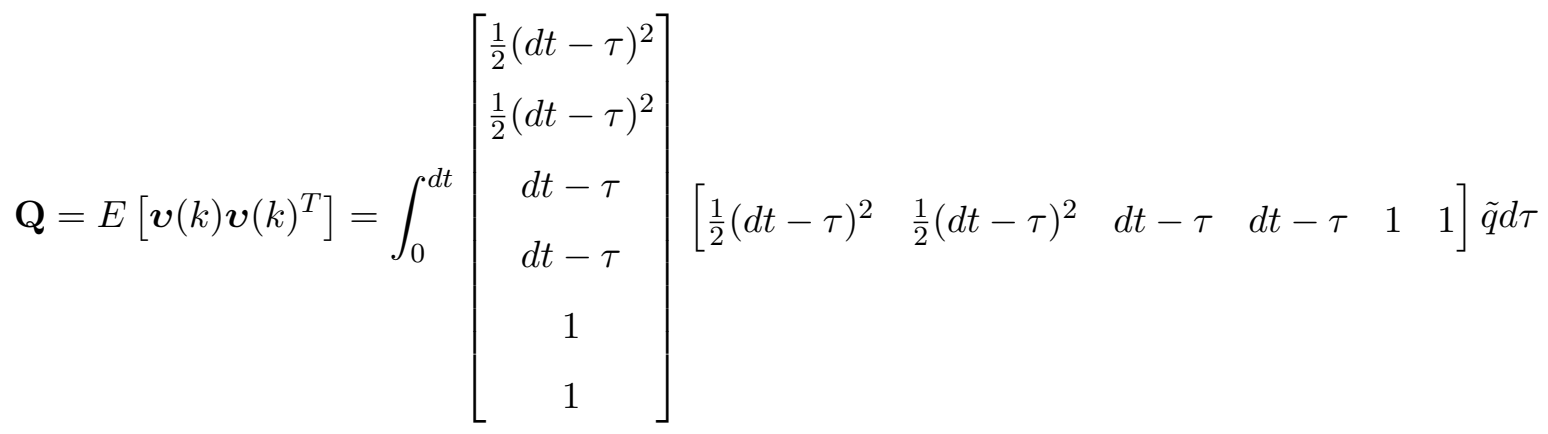

$$
\begin{aligned}
& =\tilde{q}\left[\begin{array}{cccccc}
\frac{1}{20} d t^{5} & \frac{1}{20} d t^{4} & \frac{1}{8} d t^{4} & \frac{1}{8} d t^{4} & \frac{1}{6} d t^{3} & \frac{1}{6} d t^{3} \\
\frac{1}{20} d t^{5} & \frac{1}{20} d t^{4} & \frac{1}{8} d t^{4} & \frac{1}{8} d t^{4} & \frac{1}{6} d t^{3} & \frac{1}{6} d t^{3} \\
\frac{1}{8} d t^{4} & \frac{1}{8} d t^{4} & \frac{1}{6} d t^{3} & \frac{1}{6} d t^{3} & \frac{1}{2} d t^{2} & \frac{1}{2} d^{2} \\
\frac{1}{8} d t^{4} & \frac{1}{8} d t^{4} & \frac{1}{6} d t^{3} & \frac{1}{6} d t^{3} & \frac{1}{2} d t^{2} & \frac{1}{2} d^{2} \\
\frac{1}{6} d t^{3} & \frac{1}{6} d t^{3} & \frac{1}{2} d t^{2} & \frac{1}{2} d^{2} & d t & d t \\
\frac{1}{6} d t^{3} & \frac{1}{6} d t^{3} & \frac{1}{2} d t^{2} & \frac{1}{2} d^{2} & d t & d t
\end{array}\right]
\end{aligned}
$$

To include the acceleration bias terms, Q, as defined in Equation (2.22) is expanded from 
$6 \times 6$ to $8 \times 8$ to include the discrete time GM noise variance, $\sigma_{w, b}^{2}$, presented in Equation (2.17):

$$
\mathbf{Q}=\tilde{q}\left[\begin{array}{cccccccc}
\frac{1}{20} d t^{5} & \frac{1}{20} d t^{4} & \frac{1}{8} d t^{4} & \frac{1}{8} d t^{4} & \frac{1}{6} d t^{3} & \frac{1}{6} d t^{3} & 0 & 0 \\
\frac{1}{20} d t^{5} & \frac{1}{20} d t^{4} & \frac{1}{8} d t^{4} & \frac{1}{8} d t^{4} & \frac{1}{6} d t^{3} & \frac{1}{6} d t^{3} & 0 & 0 \\
\frac{1}{8} d t^{4} & \frac{1}{8} d t^{4} & \frac{1}{6} d t^{3} & \frac{1}{6} d t^{3} & \frac{1}{2} d t^{2} & \frac{1}{2} d^{2} & 0 & 0 \\
\frac{1}{8} d t^{4} & \frac{1}{8} d t^{4} & \frac{1}{6} d t^{3} & \frac{1}{6} d t^{3} & \frac{1}{2} d t^{2} & \frac{1}{2} d^{2} & 0 & 0 \\
\frac{1}{6} d t^{3} & \frac{1}{6} d t^{3} & \frac{1}{2} d t^{2} & \frac{1}{2} d^{2} & d t & d t & 0 & 0 \\
\frac{1}{6} d t^{3} & \frac{1}{6} d t^{3} & \frac{1}{2} d t^{2} & \frac{1}{2} d^{2} & d t & d t & 0 & 0 \\
0 & 0 & 0 & 0 & 0 & 0 & \sigma_{w, b}^{2} & 0 \\
0 & 0 & 0 & 0 & 0 & 0 & 0 & \sigma_{w, b}^{2}
\end{array}\right]
$$

Assuming position, velocity, and acceleration in the different $x, y$ dimensions are not correlated, and bias is not correlated with any other parameter, the plant process noise matrix, $\mathbf{Q}$, reduces to the following:

$$
\mathbf{Q}=\tilde{q}\left[\begin{array}{cccccccc}
\frac{1}{20} d t^{5} & 0 & \frac{1}{8} d t^{4} & 0 & \frac{1}{6} d t^{3} & 0 & 0 & 0 \\
0 & \frac{1}{20} d t^{4} & 0 & \frac{1}{8} d t^{4} & 0 & \frac{1}{6} d t^{3} & 0 & 0 \\
\frac{1}{8} d t^{4} & 0 & \frac{1}{6} d t^{3} & 0 & \frac{1}{2} d t^{2} & 0 & 0 & 0 \\
0 & \frac{1}{8} d t^{4} & 0 & \frac{1}{6} d t^{3} & 0 & \frac{1}{2} d t^{2} & 0 & 0 \\
\frac{1}{6} d t^{3} & 0 & \frac{1}{2} d t^{2} & 0 & d t & 0 & 0 & 0 \\
0 & \frac{1}{6} d t^{3} & 0 & \frac{1}{2} d t^{2} & 0 & d t & 0 & 0 \\
0 & 0 & 0 & 0 & 0 & 0 & \sigma_{w, b}^{2} & 0 \\
0 & 0 & 0 & 0 & 0 & 0 & 0 & \sigma_{w, b}^{2}
\end{array}\right]
$$

Because the system is modeled with constant acceleration, the control input function, $\mathbf{u}$, in Equation (2.6) is $\mathbf{0}$.

\subsubsection{Observation Models}

The velocity, linear acceleration, and bias observation measurements are linear. Conversely, the OWTT range measurement is nonlinear, thus the measurement Equation (2.2) becomes

$$
\mathbf{z}(t)=h(\mathbf{x}(t))+\mathbf{v}(t)
$$


where $\mathrm{h}(\mathbf{x}(\mathrm{t}))$ is the nonlinear range function, discussed in more detail in the section below titled, "Range Measurement and Augmentation." In discrete time, the measurement model becomes

$$
\mathbf{z}_{k}=\mathbf{H}_{k} \mathbf{x}_{\mathbf{v}_{k}}+\tilde{\boldsymbol{v}}_{k}
$$

As shown in Equation (2.26), the observation noise, $\tilde{\boldsymbol{v}}_{k}$, is zero-mean, Gaussian white noise, such that,

$$
\tilde{\boldsymbol{v}}_{k} \sim \mathcal{N}\left(O, \mathbf{R}_{k}\right)
$$

For the measurement variance noise matrix, $\mathbf{R}$, the matrix is diagonal with the respective measurement (model velocity, acceleration, bias,or range) variance values:

$$
\begin{aligned}
& \mathbf{R}_{v e l}=\left[\begin{array}{cc}
\sigma_{v e l}^{2} & 0 \\
0 & \sigma_{v e l}^{2}
\end{array}\right] \\
& \mathbf{R}_{a c c}=\left[\begin{array}{cc}
\sigma_{a c c_{x}}^{2} & 0 \\
0 & \sigma_{a c c_{y}}^{2}
\end{array}\right] \\
& \mathbf{R}_{\text {bias }}=\left[\begin{array}{cc}
\sigma_{\text {bias }}^{2} & 0 \\
0 & \sigma_{\text {bias }}^{2}
\end{array}\right] \\
& R_{r n g}=\left[\sigma_{r n g}^{2}\right]
\end{aligned}
$$

\section{Range Measurement and Augmentation}

For each OWTT range measurement, from either the surface beacon or another vehicle, the receiving vehicle's state vector and covariance $\mathbf{P}$ matrix are augmented with the required information from the transmitting beacon. The vehicle's state vector is augmented with the transmitting beacon's pose, $\left[x_{b}, y_{b}\right]$, such that

$$
\mathbf{x}_{\text {aug }}=\left[x, y, u, v, a_{x}, a_{y}, b_{x}, b_{y}, x_{b}, y_{b}\right]^{T}
$$

The $\mathbf{P}$ matrix is augmented with the transmitting beacon's position uncertainty values, similar to the Naively Distributed Extended Kalman Filter (NEKF) [51]. Therein, the authors show that a geometry that consists of a single surface transmitting node with multiple submerged transmitting nodes (which mirrors the geometry in some of the field experiments 
presented in this thesis), the NEKF closely follows the $1 \sigma$ uncertainties of the benchmark Centralized EKF. A similar method is found in the Egocentric Extended Kalman Filter, in which the inter-vehicle ranges are assumed to be independent, and, thus the off-diagonals of the covariance matrix are zero [36]. Due to these results, algorithmic simplicity, and low acoustic bandwidth necessity, a similar comparable method for passing the transmitting beacon state uncertainties is a viable option, in which the $\mathbf{P}$ matrix is augmented in the following manner:

$$
\mathbf{P}_{\text {aug }}=\left[\begin{array}{ccc}
\boldsymbol{P}_{v} & \mathbf{0} & \mathbf{0} \\
\mathbf{0} & \sigma_{\text {beacon }}^{2} & 0 \\
\mathbf{0} & 0 & \sigma_{\text {beacon }}^{2}
\end{array}\right]
$$

$\mathbf{P}_{v}$ is the vehicle's predicted $8 X 8$ covariance matrix at time, $\mathrm{k}$, of the range measurement. $\sigma^{2}$ beacon is the sum of the $\mathrm{X}$ and $\mathrm{Y}$ position variances of the vehicle beacon transmitted in the acoustic packet. Transmitting the vehicle beacon uncertainties in this manner is more conservative than the true NEKF method and thus minimizes the potential for overconfident solutions. For the surface beacon, the GPS uncertainty is transmitted as a single value and is used as the value of $\sigma^{2}$ beacon in the matrix, $\mathbf{P}_{\text {aug }}$.

Once this augmentation process is complete, the measurement matrix, $\mathbf{H}_{\text {rng }}$ is determined to map the vehicle's state vector to the OWTT range. The OWTT range update is based upon the model presented in previous field experiments [54]. $\mathbf{x}_{\mathbf{b}}$ is the state vector of the beacon (either a surface beacon with access to GPS or another submerged AUV), such that

$$
\mathbf{x}_{\mathbf{b}}=[x, y]^{T}
$$

The range between the vehicle at time of arrival (TOA) and the beacon at time of launch (TOL) is modeled as the following:

$$
\mathbf{z}_{\mathrm{rng}}=\sqrt{\left(\mathbf{x}_{\mathbf{v}_{\mathbf{x y}}}-\mathbf{x}_{\mathbf{b}_{\mathbf{x y}}}\right)^{T}\left(\mathbf{x}_{\mathbf{v}_{\mathbf{x} \mathbf{y}}}-\mathbf{x}_{\mathbf{b}_{\mathbf{x y}}}\right)}+\boldsymbol{v}_{\mathrm{rng}}
$$

$\mathbf{x}_{\mathbf{v}_{\mathbf{x y}}}$ and $\mathbf{x}_{\mathbf{b}_{\mathbf{x y}}}$ are the XY position values of the vehicle's state at TOA and the beacon's state at TOL, respectively, and $\boldsymbol{v}_{r n g}$ is the time invariant measurement noise. Equation (2.35) rewritten in state vector form becomes

$$
\mathbf{z}_{\mathrm{rng}}=\left(\mathbf{x}^{T} \mathbf{M}^{T} \mathbf{M} \mathbf{x}\right)^{1 / 2}+\boldsymbol{v}_{\mathrm{rng}}
$$


where

$$
\begin{gathered}
\mathbf{M}=\left[\begin{array}{cc}
\boldsymbol{J}_{\mathrm{v}} & -\boldsymbol{J}_{\mathrm{b}}
\end{array}\right], \quad \boldsymbol{v}_{\mathrm{rng}} \sim \mathcal{N}\left(0, \mathbf{R}_{\mathrm{rng}}\right) \\
\mathbf{J}_{v}=\left[\begin{array}{ll}
\mathbf{I}_{2 x 2} & \mathbf{0}_{2 x 6}
\end{array}\right] \\
\mathbf{J}_{b}=\left[\mathbf{I}_{2 x 2}\right]
\end{gathered}
$$

$\mathbf{J}_{\mathrm{v}}$ and $\mathbf{J}_{\mathrm{b}}$ are defined to capture the pose information of the vehicle and the beacon at TOA and TOL, respectively. Since the range measurement is nonlinear, a Jacobian matrix of the range, evaluated at the vehicle's augmented predicted state becomes

$$
\mathbf{H}_{\mathrm{rng}_{\mathrm{k}}}=\left.\frac{\partial \mathbf{z}_{\mathrm{rng}}}{\partial \mathbf{x}}\right|_{\mathbf{x}=\hat{\mathbf{x}}_{\mathbf{a u g}_{k}}^{-}}=\left[\left(\hat{\mathbf{x}}_{\mathbf{a u g}_{k}}^{-}\right)^{T}\left(\mathbf{M}^{T}\right)(\mathbf{M})\left(\hat{\mathbf{x}}_{\mathbf{a u g}_{k}}^{-}\right)\right]^{-1 / 2}\left(\hat{\mathbf{x}}_{\mathbf{a u g}_{k}}^{-}\right)^{T}\left(\mathbf{M}^{T}\right)(\mathbf{M})
$$

The Kalman Filter measurement update equations (2.9-2.11) are then processed to update the vehicle's state with the new range measurement. The elements in the augmented state vector and augmented $\mathbf{P}$ matrix corresponding to the receiving vehicle's state variables are then saved and processed in the Kalman Filter prediction equations (2.6-2.7) for the next time step.

\section{Acceleration Bias Measurement}

With each range measurement at the TOA of an acoustic packet, the IMU EKF processes an updated state estimate, $\hat{\mathbf{x}}_{\mathbf{v}}^{+}$, from the predicted state, $\hat{\mathbf{x}}_{\mathbf{v}}^{-}$. From this update in position, an accelerometer bias in the world frame is determined as

$$
\operatorname{bias}_{a c c}=\frac{1}{2} \frac{\left(\hat{\mathbf{x}}_{\mathbf{v}_{x, y}}^{+}-\hat{\mathbf{x}}_{\mathbf{v}_{x, y}}^{-}\right) T O A}{\Delta t^{2}}
$$

where $\Delta \mathrm{t}$ is the time between the current range measurement and the previous range measurement, and $x, y$ refer to the position variables in the vehicle's state. This bias measurement is then processed in the Kalman Filter measurement update equations (2.9-2.11) to update the state's bias terms.

\footnotetext{
${ }^{0} \mathrm{~J}_{\mathrm{v}}=\left[\begin{array}{ll}\mathbf{I}_{2 \times 2} & \mathbf{0}_{2 \times 6}\end{array}\right], \mathrm{J}_{\mathrm{b}}=\mathbf{I}_{2 \times 2}$
} 


\subsubsection{Coupled Range Filter}

The IMU EKF is coupled with a range filter to prevent processing a range measurement due to inaccurate clock timing or a beacon's inaccurate state estimate. A faulty range can result from poor clock synchronization between the transmitter and the receiver or from acoustic environmental variability. A small amount of drift on either clock can change the TOL or TOA of the acoustic packet and thereby change the range calculation. Additionally, acoustic environments that contain multipath propagation or reflection can result in TOF measurements that do not resemble the slant range between the transmitting beacon and receiving vehicle, thus making the OWTT range measurement inaccurate.

When processing a range measurement, the subsequent updated state estimate can be unreliable if the transmitting beacon's state, as encoded in the acoustic packet, is inaccurate. The Jacobian OWTT range measurement-to-state mapping matrix, $\mathbf{H}_{\text {rng }}$ (Equation (2.40)), uses the transmitting beacon's state in its calculation, and this matrix is then used to update the receiving vehicle's state with the innovation in Equation (2.10). Therefore, any inaccuracy in the transmitting beacon's state estimate can easily contribute to an error in the receiving vehicle's state estimate as soon as the OWTT range is processed. To address these issues, the coupled range filter in the IMU EKF uses two different processes to prevent the IMU EKF from processing a faulty range measurement.

The coupled range filter in the IMU EKF uses two different processes to prevent the IMU EKF from processing a faulty range measurement or an inaccurate beacon's position. These two processes are a range threshold filter and a state acceptance gate, both summarized in Algorithm 2.

The first decision point of the coupled range filter is the range threshold filter, which prevents the IMU EKF from processing the measurement update Equations (2.9 - 2.10) on a faulty range. Simply, if the OWTT range is less than the set maximum threshold value, the range is discarded, and the measurement update equations (2.9-2.11) are not processed. The range threshold value can be determined in a variety of methods. Examples include geographic constraints or known acoustic range limitations based on environment or equipment.

After the vehicle's updated state is determined, a state acceptance gate (analogous to a velocity filter) is applied to prevent processing a receiving vehicle's updated state estimate 


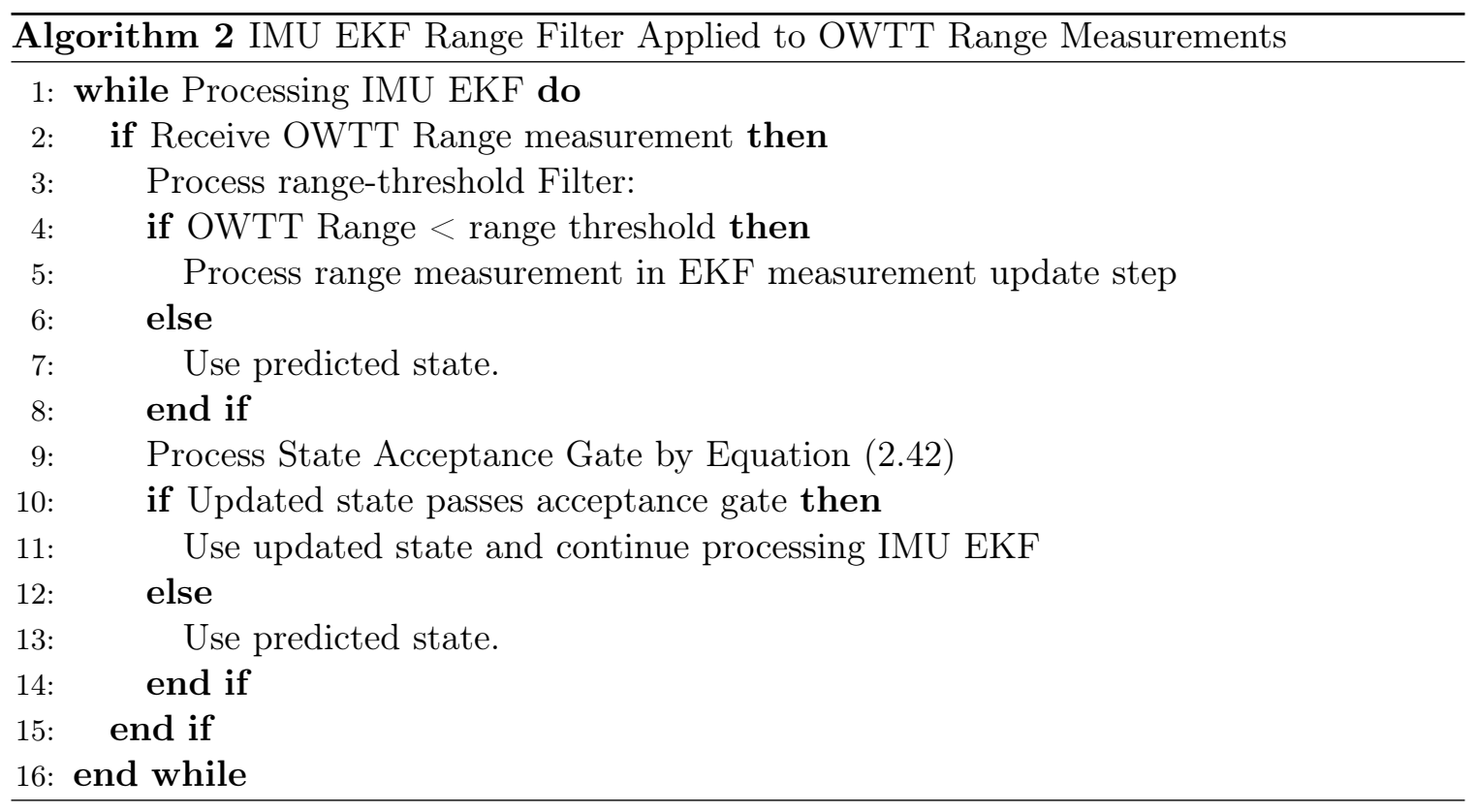

because of an inaccurate beacon's state estimate. The IMU EKF tracks its previous state estimated position and time of the last OWTT range measurement from any beacon. After determining the updated state, the IMU EKF calculates a distance from the vehicle's state position at the time of the last range measurement to the current vehicle's updated state position. This distance is then divided by the time since the last OWTT range update. This calculation results in a speed, which is then compared to predetermined speed value. If the calculated speed is less than this maximum value, the updated state is processed and an acceleration bias measurement is determined. If the calculated speed is greater than predetermined speed value, the updated state estimate is disregarded, and the vehicle's predicted state is propagated forward. Equations (2.42-2.43) show the speed calculation, where $x, y$ are the vehicle's estimated position coordinates in the world frame, and $k, t$ are the current time and last range update time, respectively.

$$
\begin{gathered}
\frac{\sqrt{\left(x_{k}-x_{t}\right)^{2}+\left(y_{k}-y_{t}\right)^{2}}}{\Delta t}<\text { Speed Value } \\
\Delta t=k-t
\end{gathered}
$$

The speed value used can be determined by the vehicle's maximum rated speed or the mission's maximum commanded speed with a deviation. For example, if the mission's commanded speed is 2.5 knots, and the vehicle is using its DVL for speed measurements, the 
speed value for the state acceptance gate could be set to 3.0 knots, which provides a $20 \%$ deviation to allow for any variability in the DVL measurements.

\subsection{Attitude Complementary Filter}

The MEMS IMU not only provides odometry input from its linear accelerations, but it also provides measurements for attitude estimation. Roll, pitch, and yaw are determined from the IMU's accelerometer, gyroscope, and magnetometer measurements. The accelerometer and magnetometer measurements provide a high frequency source of attitude determination, and the gyroscope measurements determine a low frequency source of attitude. In this case, since attitude measurements can be considered a signal determined by two different noisy measurements (one that is high frequency and the other that is low frequency), a complementary filter can be used to reduce the error [6].

The complementary filter uses a combination of a low-pass filter and high-pass filter to refine the attitude estimation. The low-pass filter allows the low frequency components to pass through, while the high-pass filter allows the high frequency components to pass through. In essence, the complementary filter cancels the long-term (low-frequency) drift from the gyroscope measurements to get a more accurate determination of attitude.

As noted before, attitude is determined from two sources: (1) accelerometer and magnetometer, and (2) gyroscopes. From the accelerometer and magnetometer measurements, roll $(\phi)$, pitch $(\theta)$, and yaw $(\psi)$, are determined:

$$
\begin{gathered}
\phi=\operatorname{atan} 2\left(-a_{y},-a_{z}\right) \\
\theta=\operatorname{atan} 2\left(a_{x}, \sqrt{a_{y}^{2}+a_{z}^{2}}\right) \\
\psi=\operatorname{atan} 2\left(-{ }^{l} m_{y},-{ }^{l} m_{x}\right)-\psi_{o}
\end{gathered}
$$

where $a$ is the respective accelerometer measurement, $\psi_{0}$ is the local magnetic declination, and ${ }^{l} m$ is the respective magnetometer measurement rotated to the local frame [34].

The model for the complementary filter is as follows:

$$
\Theta=\alpha *(\Theta+\omega * d t)+(1-\alpha) * \Theta_{M a g} / A c c
$$


$\Theta$ is the vector of roll, pitch, and yaw, that is, $\Theta=[\phi, \theta, \psi]^{\mathrm{T}}, \omega$ is the angular velocity measured by the IMU gyroscopes, $\Theta_{\mathrm{Mag} / \mathrm{Acc}}$ is the attitude vector calculated by equations (2.44-2.46), and $\alpha$ is the filter coefficient [27].

The IMU's magnetometer was calibrated by the nominal geometric calibration procedure [8], and the complementary filter was adjusted by comparing the output to the onboard OceanServer compass that was used by the vehicle when the data sets were recorded. The complementary filter was not used for attitude estimation for the SandShark field trials due to the inability to calibrate the IMU magnetometer. 
THIS PAGE INTENTIONALLY LEFT BLANK 


\section{Chapter 3}

\section{Experiment Configuration}

\section{$3.1 \quad$ Vehicles}

OceanServer Technology, Inc. Iver2 vehicles and a Bluefin Robotics SandShark micro-autonomous underwater vehicle ( $\mu \mathrm{AUV})$ were used during the field trials reported in this thesis. Details of these two platforms as well as instrumentation are discussed in detail in this chapter.

\subsubsection{OceanServer Technology, Inc. Iver2 AUV}

Two Iver2 AUVs [11], Iver-106 and Iver-136, were used during the field trials conducted for this research. These two vehicles are featured in Figure 3-1 on board the R/V Shana Rae for the "Satellites to the Ocean Floor" research project [44]. Iver-106 was equipped with a Woods Hole Oceanographic Institution (WHOI) 25-kHz acoustic micro-modem [24], a SonTek Doppler Velocity Log (DVL) for speed estimation, a MEMS OceanServer compass for attitude estimation, a depth sensor, a GPS receiver, and a Pololu AltIMU-10 v5 MEMS IMU. This IMU consisted of a triaxial gyroscope, accelerometer, compass, and altimeter (LSM6DS33, LIS3MDL, and LPS25H Carrier). The other vehicle, Iver-136, had a similar equipment configuration with a WHOI acoustic modem, compass, a GPS receiver, and depth sensor. Additionally, the vehicle was equipped with a dual upward/downward tracking 600 kHz RDI DVL and a Microstrain 3DM-GX3-25 MEMS IMU. Figure 3-2 shows the systems configuration layout on the vehicles. Each of the vehicles' software was modified to run Linux Ubuntu (Version 14.04) with the vehicle's original Windows based computer operating as a virtual machine. This modification enabled compatibility with WHOI's Deep Submergence 


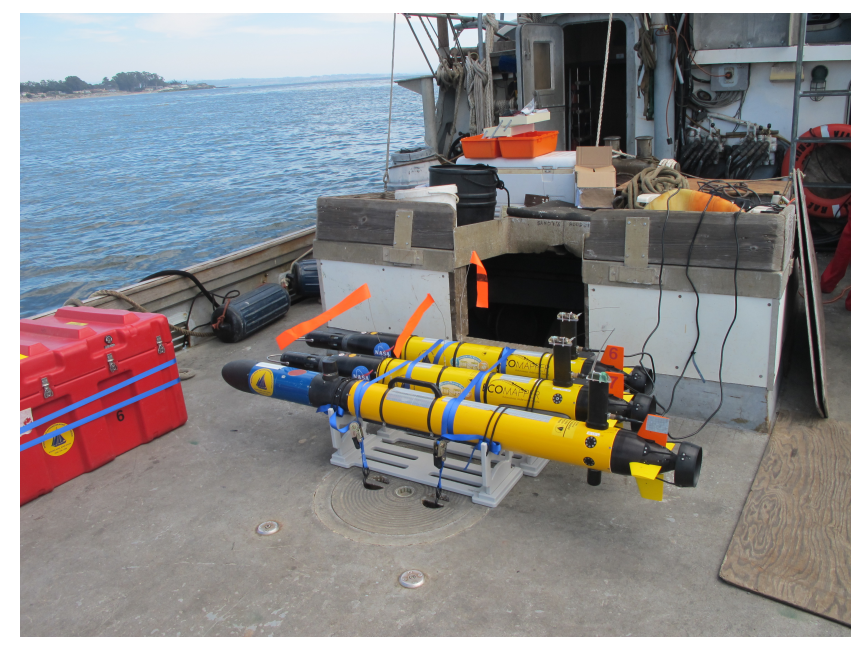

Figure 3-1: OceanServer Iver2 AUVs onboard the R/V Shana Rae. Front to Back: Iver-136, Iver-107, Iver-106. Photo courtesy of Dr. David Fratatoni during the KISS Field Project [44] in 2016.

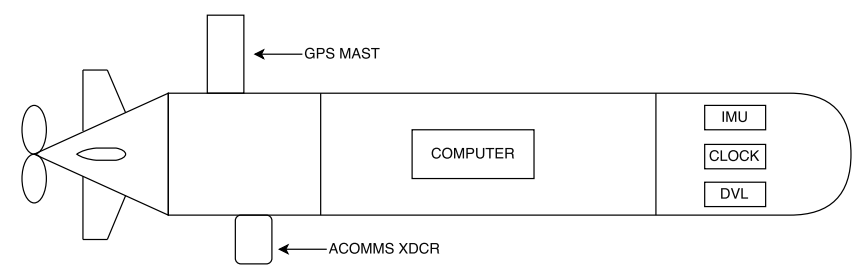

Figure 3-2: Iver2 AUV Systems Configuration Layout: Displays the main components and sensors necessary for the IMU EKF navigation method.

Lab acoustic communications (ACOMMS) software.

Bench tests were conducted to determine the IMU's static accelerometer and gyrocompass biases. Each vehicle operated its normal operating hotel load throughout the duration of the test; Iver-106 ran for approximately 19.1 hours, and Iver-136 ran for 15.6 hours. Table 3.1 summarizes the statistics for both IMUs. In this table, acc is defined as the respective accelerometer, and gyro is defined as the respective gyroscope. The accelerometer and gyroscope biases were subtracted from the raw measurements prior to being infused into the IMU EKF and the complementary filter. The accelerometer standard deviations were used in the measurement noise matrix, $\mathbf{R}$.

\subsubsection{Bluefin Robotics SandShark AUV}

The Bluefin Robotics SandShark used in these field experiments was a small, low- $\mu$ AUV that was configured with a payload suitable for acoustic navigation [48]. Specifically, this vehicle 
Table 3.1: Pololu \& Microstrain IMU Accelerometer and Gyrocompass Statistics

(a) Iver-106 Pololu IMU

\begin{tabular}{|c|c|c|}
\hline Sensor & Static Bias, $\mu$ & Standard Deviation, $\sigma$ \\
\hline$a c c_{x}$ & $29.95 \mathrm{mg}$ & $0.945 \mathrm{mg}$ \\
\hline$a c c_{y}$ & $5.744 \mathrm{mg}$ & $0.967 \mathrm{mg}$ \\
\hline$a c c_{z}$ & $19.45 \mathrm{mg}$ & $1.204 \mathrm{mg}$ \\
\hline gyro $_{x}$ & $2.129582 \mathrm{deg} / \mathrm{s}$ & $0.030719 \mathrm{deg} / \mathrm{s}$ \\
\hline gyro $_{y}$ & $-2.882762 \mathrm{deg} / \mathrm{s}$ & $0.033401 \mathrm{deg} / \mathrm{s}$ \\
\hline gyro $_{z}$ & $-5.167089 \mathrm{deg} / \mathrm{s}$ & $0.042471 \mathrm{deg} / \mathrm{s}$ \\
\hline
\end{tabular}

(b) Iver-136 Microstrain IMU

\begin{tabular}{|c|c|c|}
\hline Sensor & Static Bias, $\mu$ & Standard Deviation, $\sigma$ \\
\hline$a c c_{x}$ & $17.587 \mathrm{mg}$ & $0.631 \mathrm{mg}$ \\
\hline$a c c_{y}$ & $15.473 \mathrm{mg}$ & $0.682 \mathrm{mg}$ \\
\hline$a c c_{z}$ & $4.782 \mathrm{mg}$ & $0.707 \mathrm{mg}$ \\
\hline gyro $_{x}$ & $0.001032 \mathrm{deg} / \mathrm{s}$ & $0.002490 \mathrm{deg} / \mathrm{s}$ \\
\hline gyro $_{y}$ & $-0.000384 \mathrm{deg} / \mathrm{s}$ & $0.002270 \mathrm{deg} / \mathrm{s}$ \\
\hline gyro $_{z}$ & $0.004779 \mathrm{deg} / \mathrm{s}$ & $0.002408 \mathrm{deg} / \mathrm{s}$ \\
\hline
\end{tabular}

was equipped with a 9 degrees of freedom MEMS IMU collocated on an Android phone device computer board, an altimeter, a depth sensor, an acoustic micro-modem, and a GPS receiver. A unique payload configuration for this particular SandShark was the tetrahedral hydrophone array used for its acoustic communications, as shown in Figure 3-3.

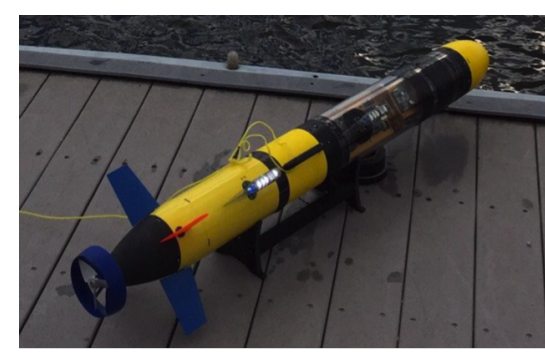

(a) SandShark $\mu \mathrm{AUV}$

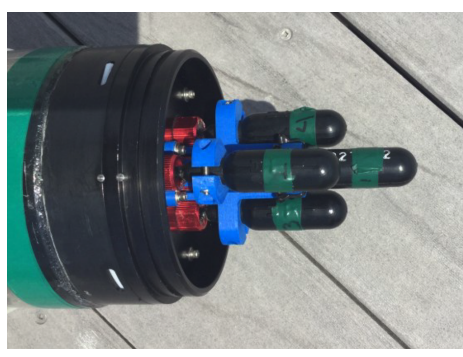

(b) SandShark Tetrahedral hydrophone array.

Figure 3-3: Bluefin Robotics SandShark $\mu$ AUV. [Left] SandShark $\mu$ AUV before field trials conducted on the Charles River in Cambridge, Massachusetts. [Right] Nose cone removed to display the acoustic tetrahedral hydrophone array. Pictures from proof of concept trails of the acoustic navigation payload [48]. 


\subsection{Acoustic Communications}

\subsubsection{Iver2 AUVs Communications}

For the acoustic communications (ACOMMS) with the Iver2 vehicles, both the topside node and submerged nodes ran their own separate software on Ubuntu Linux computers, similar to the network in previous successful experiments [52]. Figure 3-4 shows an overall block diagram of the acoustic communications network for both the topside and vehicle nodes.

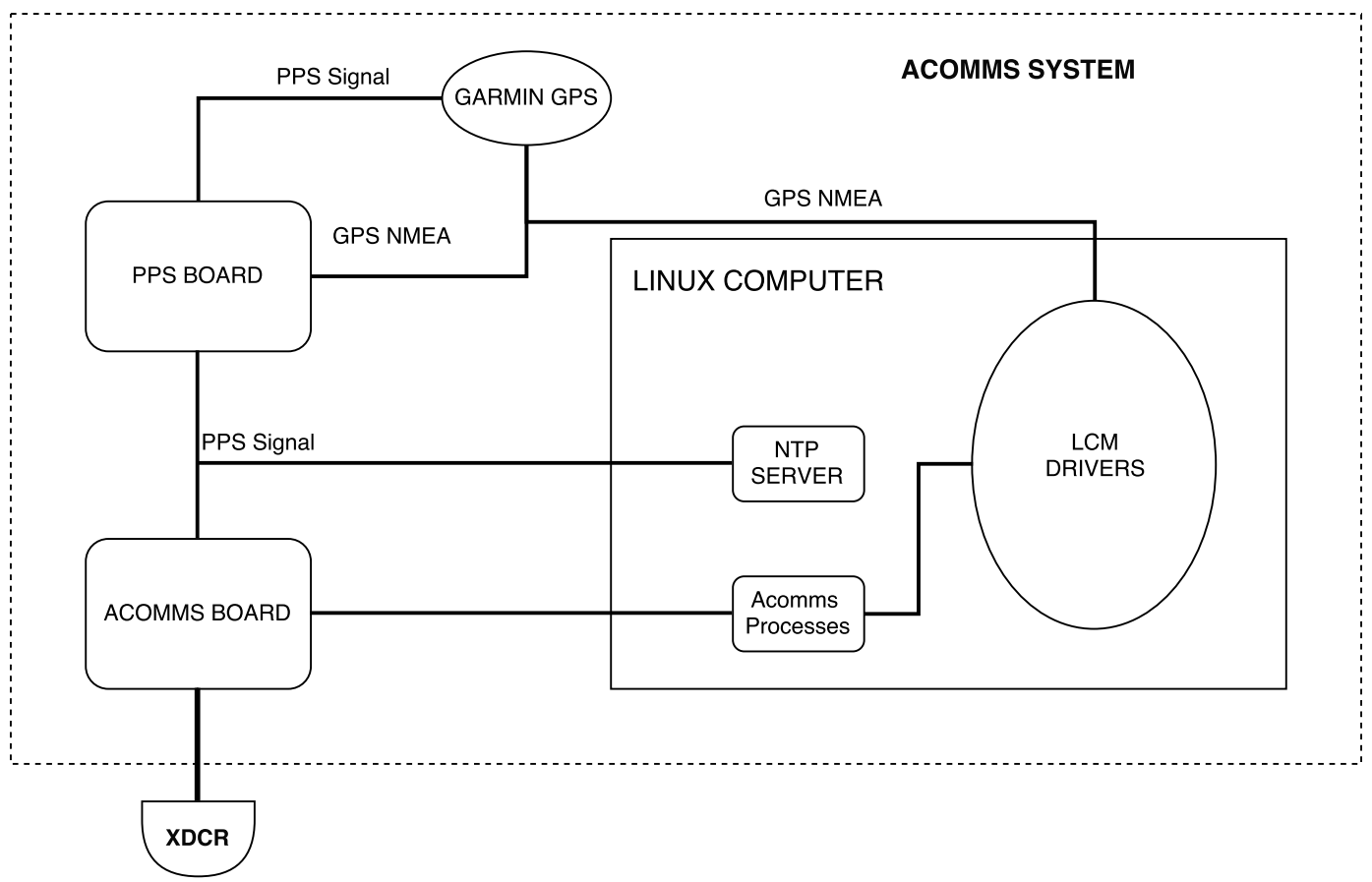

Figure 3-4: Acoustic Communication System Block Diagram: Displays the acoustic communications system configuration for both the topside and vehicle nodes.

Each of the nodes used a PPSBOARD [17, 18], which included a SeaScan, Inc. temperaturecompensated crystalline oscillator to serve as a precision clock reference. The PPSBOARD processed both the PPS signal and the GPS National Marine Electronic Association (NMEA) strings and transmitted a PPS signal to the node's computer to serve as the network time protocol (NTP) server. Additionally, the PPSBOARD sent a PPS signal to the ACOMMS acoustic modem board for precision timing of transmission or reception of acoustic packets. The timing synchronization between all of the node's NTP servers was less than 1 millisecond and was not observed to drift beyond this error throughout the field trials. Therefore, assuming a sound speed of $1500 \mathrm{~m} / \mathrm{s}$, the timing error resulted in range errors of less than 1.5 meters. 
For these field experiments, a 20 foot rigid hull inflatable skiff served as the topside beacon carrying the necessary software and hardware for ACOMMS. The necessary software ran on its own independent laptop computer, which was connected to the hardware interfaces and to a transducer. This transducer was lowered over the side for the duration of the experiments.

All three of the nodes operated on a fixed time division multiple access (TMDA) cycle period of 60 seconds. The topside node transmitted at the top of each minute (:00), Iver-106 transmitted at time :10, and Iver-136 transmitted at time :30. Thus, each vehicle received two OWTT ranges in a one minute timing window assuming perfect ACOMMS message receipt.

\subsubsection{SandShark $\mu$ AUV Acoustic Communications}

The original intent of this SandShark configuration with the tetrahedral array was for inverted ultra-shortbase-line OWTT navigation [30, 40]. However, for the purpose of this thesis, this platform used the tetrahedral array for OWTT range measurements. Instead of a PPSBOARD, the SandShark contained a Microsemi SA.45 chip scale atomic clock (CSAC) for precision time keeping and synchronization [19, 25]. Each of the four elements of the acoustic array processed a range estimate by a matched filtering process, and these resulting ranges were the raw measurements for the IMU EKF. Because each element processed a range, the median of these four ranges was used as the range measurement in the IMU EKF.

A topside acoustic source was lowered dock side in the Charles River during these experiments. This source transducer was syncrhonized with a GPS PPS signal and transmitted its GPS location and TOL, which was then decoded by the SandShark. During these experiments, the topside source transmitted at a frequency of $1 \mathrm{~Hz}$.

\subsection{IMU EKF Implementation}

During the field experiments for this thesis, the Lightweight Communications and Marshalling (LCM) [28] system for the Iver2 vehicles and a similar system on the SandShark vehicle recorded data from all of the various sensors. Using Matlab, the IMU EKF navigation method (described in detail in Chapter 3) processed the time-sequential data from 
the field experiments. The results from this real-time implementation of the IMU EKF in Matlab are those used for analysis in this thesis. 


\section{Chapter 4}

\section{Field Results}

A series of trials were conducted to validate the IMU EKF navigation solution. These trials consisted of different platforms, distinct acoustic environments, and diverse mission trajectories and configurations in order to present performance summaries with experimental diversity. The first three experiments were conducted in controlled environments of local waters, and the last experiment provides results from an open ocean environment.

The first analysis of the October 2016 Iver2 field experiments proves the effectiveness of the bias estimation in the IMU EKF and the use of multiple transmitting OWTT beacons. The second field experiment, consisting of the SandShark $\mu$ AUV on the Charles River, offered a distinct platform and mission configuration and demonstrates the capability of the coupled range filter. The third experiment conducted on the Iver2 vehicles in April 2017 in Ashumet Pond confirms the capability of the coupled range filter in a multi-vehicle trajectory that is indicative of current real-world AUV missions. Lastly, the open water experiment was conducted with one of the Iver2 vehicles in Monterey Bay, CA, providing performance results of this navigation method in open ocean currents. Details of these experiments and the associated analyses are discussed in this chapter.

A variety of navigation solutions are presented for comparison in all of the following analyses. Details of the solutions are discussed in each individual experiment section, and Table 4.1 provides a summarized description for each solution. Optimization of the CL EKF was not analyzed for this thesis. 
Table 4.1: Navigation Solution Description Summary

\begin{tabular}{|c|c|c|}
\hline Solution Type & Description & Inputs \\
\hline IMU EKF & $\begin{array}{l}\text { Constant acceleration model, } \\
\text { Includes bias estimation, } \\
\text { Includes coupled range filter }\end{array}$ & $\begin{array}{l}\text { Linear accelerations, } \\
\text { Model Velocity or DVL, } \\
\text { TOF measurements }\end{array}$ \\
\hline DR EKF & $\begin{array}{l}\text { Constant acceleration model, } \\
\text { No bias estimation, No TOF measurements }\end{array}$ & $\begin{array}{l}\text { Linear accelerations, } \\
\text { Model Velocity or DVL }\end{array}$ \\
\hline CL EKF (Iver2) & $\begin{array}{c}\text { Constant velocity model, } \\
\text { Includes TOF measurements, } \\
\text { No range filtering }\end{array}$ & $\begin{array}{l}\text { Model Velocity or DVL, } \\
\text { TOF measurements, } \\
\text { GPS (April } 2017 \text { Only) }\end{array}$ \\
\hline SS CL (SandShark) & $\begin{array}{l}\text { Model-velocity aided dead-reckoned, } \\
\text { No TOF measurements }\end{array}$ & $\begin{array}{l}\text { Dynamic model-velocity, } \\
\text { GPS }\end{array}$ \\
\hline
\end{tabular}

\subsection{Ashumet Pond Trials - October 2016}

The first set of field experiments used Iver-106 and Iver-136 on Ashumet Pond in Falmouth, MA on October 25, 2016. Ashumet Pond is a small, local, 203 acre natural kettle-hole pond with a maximum depth of approximately 20 meters and an average depth of approximately 7 meters [1].

The closed-loop navigation solution (referred to as the CL EKF) used onboard the vehicles during the execution of these field trials was a continuous white noise acceleration model [4] (i.e., a kinematic constant-velocity model) EKF that processed OWTT range measurements from both the topside beacon and the other vehicle. For the CL EKF speed measurements, Iver-106 used its DVL, and Iver-136 used its dynamic model velocity - its DVL was not operational during these experiments. The IMU EKF presented in this thesis is a real-time implementation of the data gathered during this field experiment, and the speed measurements are defined specifically for each analysis. Also presented is a dead-reckoned EKF (referred to as the DR EKF), which is the same solution as the IMU EKF but did not incorporate any of the OWTT ranges nor the bias estimation.

\subsubsection{Trajectory Summary}

For these field experiments, both of the AUVs operated on the surface throughout the trials to allow for GPS to serve as the source of ground-truth position, but GPS was not implemented into any of three navigation solutions except for initialization. After both vehicles executed their initialization sequences, Iver-136 was deployed first, then Iver-106 
was deployed, followed by the surface beacon skiff getting underway. Both of the vehicles executed a square pattern trajectory consisting of cardinal headings with Iver-106 operating to the north of Iver-136. Data was trimmed to the time line of the experiment, and no OWTT range measurements were removed from the field data for the real-time implementation of the IMU EKF except for the initial two ranges to Iver-106 to prevent a singularity (details discussed in Section 4.4.2). Iver-106 experienced approximately $26.5 \%$ acoustic packet loss, and Iver-136 experienced $32.6 \%$ acoustic packet loss.

\subsubsection{Performance Analysis}

The IMU EKFs for both vehicles contained parameter values that are presented in Table 4.2 .

Table 4.2: Vehicle IMU EKF Parameter Values

(a) Iver-106 IMU EKF

\begin{tabular}{|c|c|}
\hline Parameter & Value \\
\hline$d t$ & $.037 \mathrm{~s}$ \\
$\tilde{q}$ & 1.0 \\
$\sigma_{b}$ & $1.0 \mathrm{~m} / \mathrm{s}^{2}$ \\
$\sigma_{\text {vel }}$ & $1.0 \mathrm{~m} / \mathrm{s}$ \\
$\sigma_{a c c_{x}}$ & $0.945 \mathrm{mg}$ \\
$\sigma_{\text {acc }}$ & $0.976 \mathrm{mg}$ \\
$\sigma_{\text {bias }}$ & $1.0 \mathrm{~m} / \mathrm{s}^{2}$ \\
$\sigma_{r n g}$ & $10 \mathrm{~m}$ \\
$T_{c_{x}}$ & $100 \mathrm{~s}$ \\
$T_{c_{y}}$ & $500 \mathrm{~s}$ \\
\hline
\end{tabular}

(b) Iver-136 IMU EKF

\begin{tabular}{|c|c|}
\hline Parameter & Value \\
\hline$d t$ & $0.039 \mathrm{~s}$ \\
$\tilde{q}$ & 1.0 \\
$\sigma_{b}$ & $1.0 \mathrm{~m} / \mathrm{s}^{2}$ \\
$\sigma_{\text {vel }}$ & $1.0 \mathrm{~m} / \mathrm{s}$ \\
$\sigma_{a c c_{x}}$ & $0.631 \mathrm{mg}$ \\
$\sigma_{a c c_{y}}$ & $0.682 \mathrm{mg}$ \\
$\sigma_{\text {bias }}$ & $1.0 \mathrm{~m} / \mathrm{s}^{2}$ \\
$\sigma_{r n g}$ & $10 \mathrm{~m}$ \\
$T_{c_{x}}$ & $100 \mathrm{~s}$ \\
$T_{c_{y}}$ & $500 \mathrm{~s}$ \\
\hline
\end{tabular}

The overall mission trajectory for both vehicles is presented in Figure 4-1. Iver-106 started on an easterly heading, turned north, east, south, and then headed west back towards the start point. Similarly, Iver-136 proceeded easterly, then turned to the south, then westerly, and made its final turn to the north to complete the box trajectory before heading west towards its start point.

On both plots, the IMU EKF (green) closely tracked the GPS position (blue) and outperforms the CL EKF solution (light blue). As stated earlier, the Iver-106 CL EKF used its DVL as the means of speed estimation, and the IMU EKF (with a dynamic model velocity for speed estimation) still outperformed the CL EKF by an average error from GPS position 


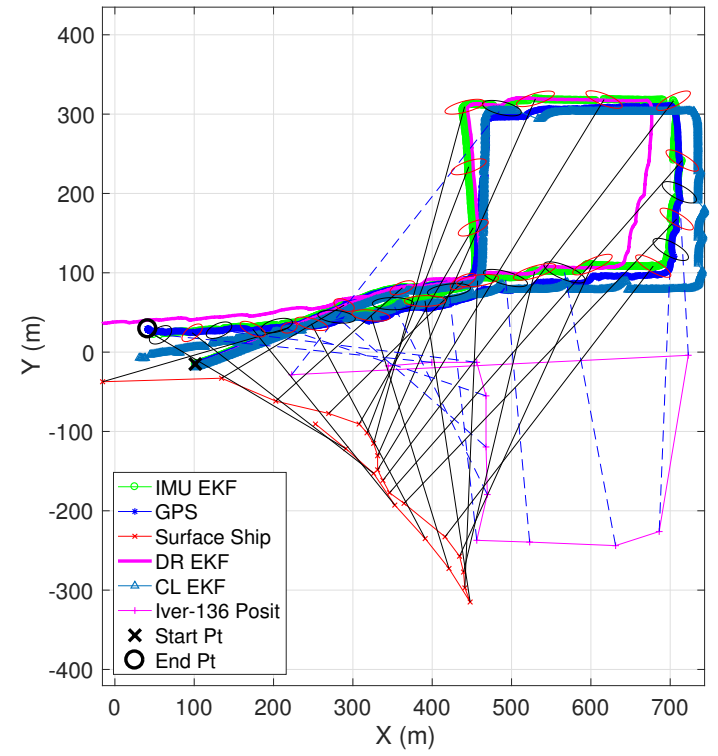

(a) Iver-106 Position Summary

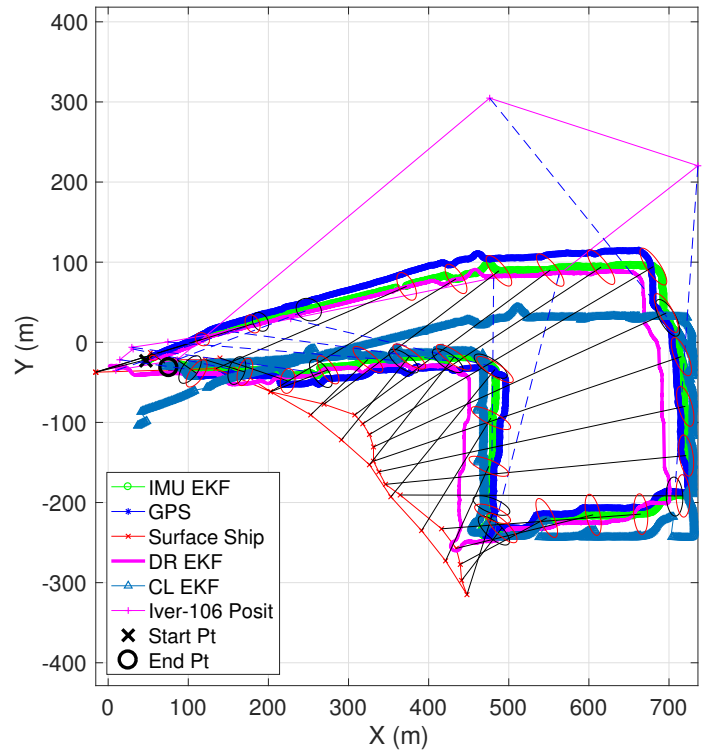

(b) Iver-136 Position Summary

Figure 4-1: Iver2 Position Summary. [Left] Iver-106 Trajectory. [Right] Iver-136 Trajectory. For both plots: GPS (Blue), IMU EKF (Green), CL EKF (Light Blue), DR EKF (Magenta). Black lines are ranges from the topside beacon, and blue dashed lines are ranges from the other AUV. Red ellipses are IMU EKF position uncertainties associated with a topside range, and block ellipses are IMU EKF uncertainties associated with a range from the other AUV. The IMU EKF for both vehicles is model velocity-aided. For Iver-106, the CL EKF is DVL-aided. For Iver-136, the CL EKF is model velocity-aided.

of 10.26 meters to 20.8 meters, respectively. For Iver-136, the CL EKF used a model velocity for speed estimation, and, as Figure 4-1b depicts, the IMU EKF outperformed the other solutions as well. Iver-136 possesses the large initialization error that was due to the vehicle receiving a OWTT range measurement from Iver-106 prior to both vehicles commencing their missions. Additionally, Iver-136 used a model velocity of $1.23 \mathrm{~m} / \mathrm{s}$ versus $1.03 \mathrm{~m} / \mathrm{s}$ (used in the IMU EKF), which more accurately matched the actual speed of the vehicle during the field trials. These two discrepancies account for the majority of the error in the Iver-136 CL EKF solution. A summary of the average errors for all three solutions for both vehicles is presented in Table 4.3.

Time series plots of the EKF error summaries are presented in Figures 4-2 and 4-3 for both vehicles. As shown for both vehicles, the IMU EKF error outperforms the other EKF methods throughout the majority of the field trials. On Iver-106 from 1200 to 1400 seconds, the steady rise in the error was due to its northern leg trajectory. On this leg, Iver-106 received three range updates only from the surface beacon and no range measurements from 
Table 4.3: Vehicle EKF Average Error from GPS Summary

\begin{tabular}{|c|c|c|}
\hline Solution & Iver-106 & Iver-136 \\
\hline IMU EKF & $10.26 \mathrm{~m}$ & $12.95 \mathrm{~m}$ \\
\hline CL EKF & $20.82 \mathrm{~m}$ & $50.21 \mathrm{~m}$ \\
\hline DR EKF & $42.03 \mathrm{~m}$ & $36.33 \mathrm{~m}$ \\
\hline Distance Traveled & $1.73 \mathrm{~km}$ & $1.91 \mathrm{~km}$ \\
\hline
\end{tabular}

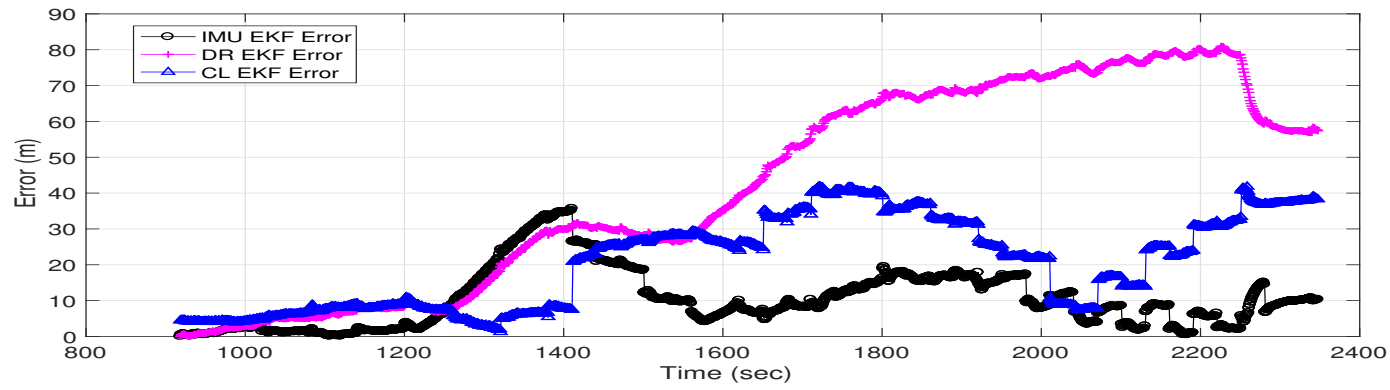

(a) Iver-106 Error Summary

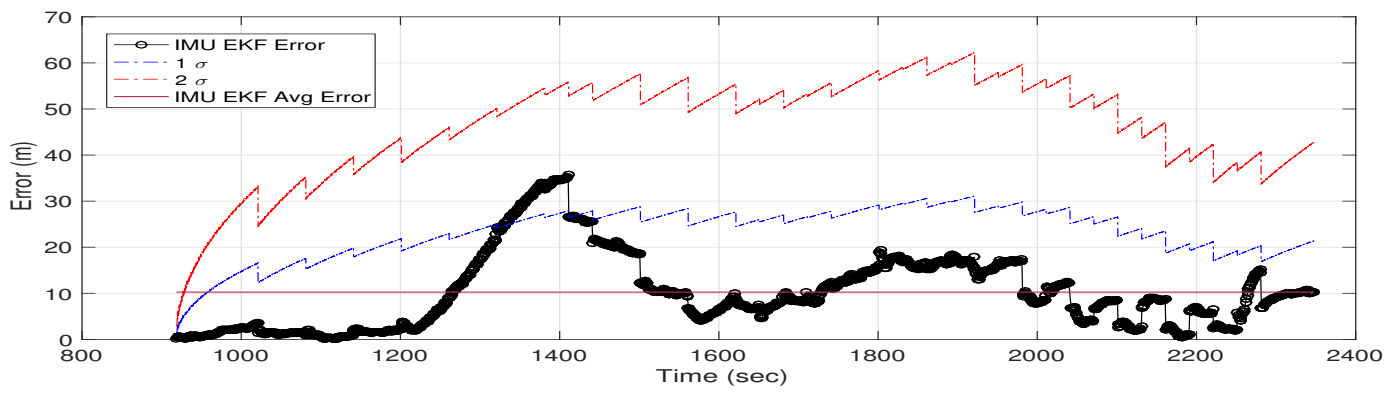

(b) Iver-106 IMU EKF Error

Figure 4-2: Iver-106 EKF Performance. [Top] Time series error plots of DR EKF (magenta), CL EKF (Blue), and IMU EKF (Black). [Bottom] Time series error plot of IMU EKF with associated $1 \sigma$ uncertainty (blue) and $2 \sigma$ uncertainty (red) lines. Error is the distance of the solution's estimated position from the GPS position.

Iver-136. Since the surface beacon was mostly to the south of Iver-106, only the Y position of Iver-106 was constrained, and its X position continued to grow. However, after Iver106 turned to its easterly leg, the IMU EKF quickly reconverged after receiving additional OWTT ranges and maintained its error below the $2 \sigma$ line for the rest of the field trial. Iver136 IMU EKF exhibited similar performance with respect to the other EKF solutions. Even though the CL EKF has poor initialization due to it processing the poor quality range from Iver-106, the IMU EKF still displayed better performance after the CL EKF reconverged at 1800 seconds. 


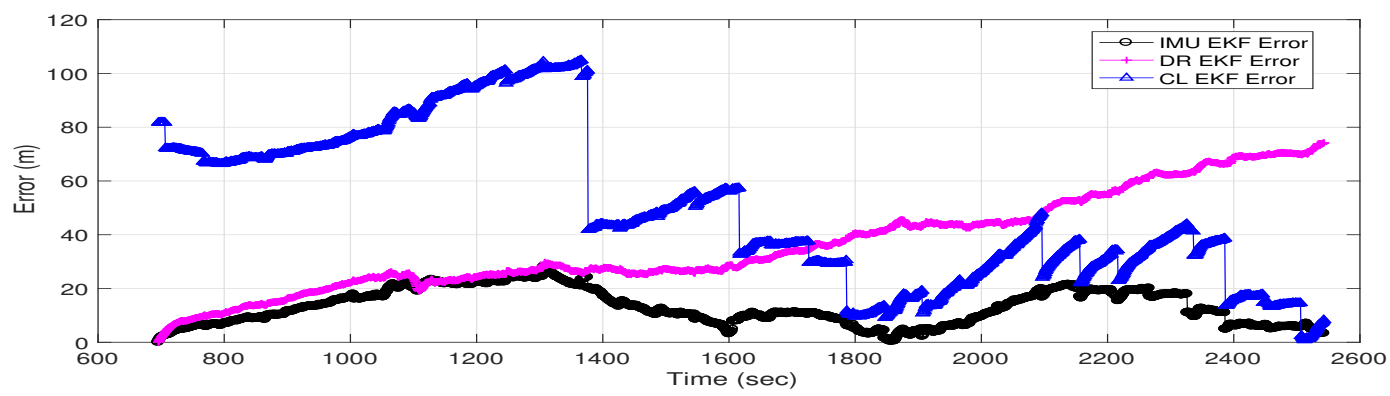

(a) Iver-136 Error Summary

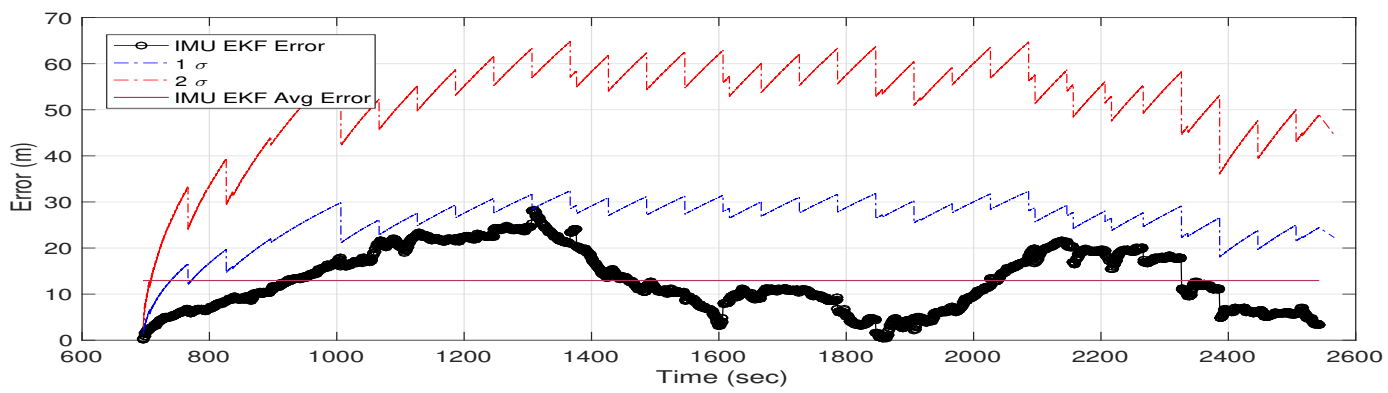

(b) Iver-136 EKF Error

Figure 4-3: Iver-136 IMU EKF Performance. [Top] Time series error plots of DR EKF (magenta), CL EKF (Blue), and IMU EKF (Black). [Bottom] Time series error plot of IMU EKF with associated $1 \sigma$ uncertainty (blue) and $2 \sigma$ uncertainty (red) lines. Error is the distance of the solution's estimated position from the GPS position.

\section{IMU EKF DVL \& Model Velocity Comparison}

A useful criterion to determine the accuracy of the dynamic model velocity-aided solution is to compare the IMU EKF solution with DVL as the input for speed estimation versus the model velocity. To achieve this comparison, the IMU EKF for Iver-106 was reprocessed with DVL measurements, and all parameters shown in Table 4.2 remained the same except the parameters presented in Table 4.4. These parameters were changed to properly tune the IMU EKF with the DVL input.

Table 4.4: DVL-aided IMU EKF Parameter Values

\begin{tabular}{|c|c|}
\hline Parameter & Value \\
\hline$\tilde{q}$ & 0.1 \\
$\sigma_{\text {vel }}$ & $1.0 \mathrm{~m} / \mathrm{s}$ \\
\hline
\end{tabular}

As expected, the DVL-aided IMU EKF reduced the average position error by approximately $12.9 \%$. Additionally, the DVL-aided IMU EKF exhibited a $1 \sigma$ position uncertainty of about one-third the value of the model velocity-aided IMU EKF, as shown by comparing 
the $1 \sigma$ cure in Figure 4-5 to the model velocity-aided IMU EKF $1 \sigma$ curve presented in Figure 4-2b. Also, as expected, the DVL-aided IMU EKF DR solution reduced its average error by $16.4 \%$ from the model velocity-aided IMU EKF error. Both of the IMU EKFs (with and without the DVL) exhibited good performance since both of the error lines remained under the $2 \sigma$ position uncertainty curves the entire length of the field trials. Figure $4-4$ shows the position summary plot for the EKFs using the DVL, and Figure 4-5 shows the time series error performance. The average error results comparing the various EKFs with and without the DVL are summarized below in Table 4.5. The "N/A" result for the CL EKF using the model velocity is because the CL EKF used the DVL for its speed measurement during these field trials.

Table 4.5: Iver-106 EKF Performance Summary

\begin{tabular}{|c|c|c|}
\hline Solution & DVL Aided & Model velocity-aided \\
\hline IMU EKF & $8.94 \mathrm{~m}$ & $10.26 \mathrm{~m}$ \\
\hline CL EKF & $20.82 \mathrm{~m}$ & $\mathrm{~N} / \mathrm{A}$ \\
\hline DR EKF & $35.13 \mathrm{~m}$ & $42.03 \mathrm{~m}$ \\
\hline
\end{tabular}

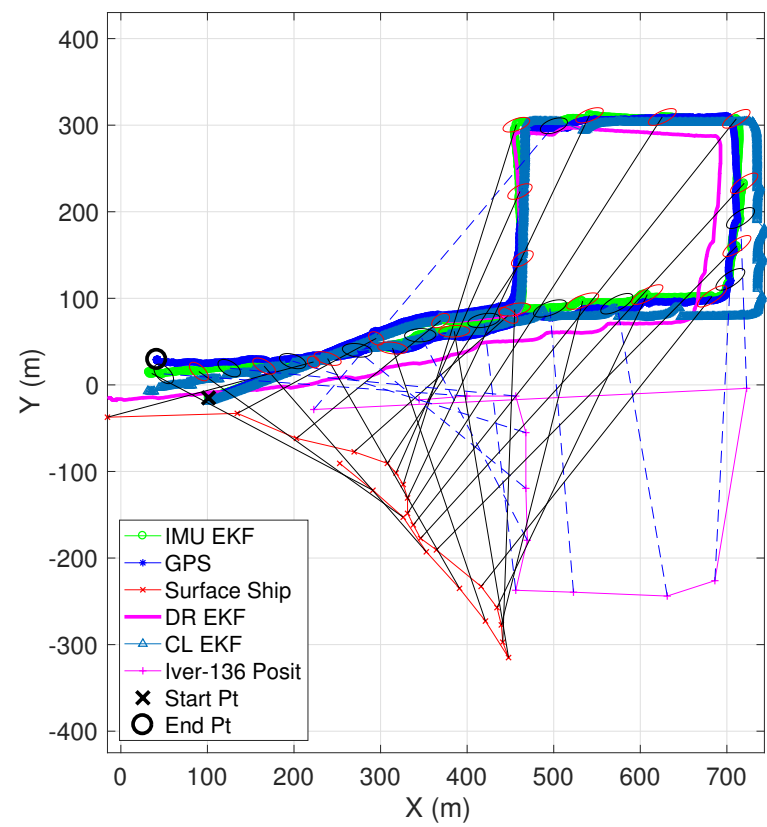

Figure 4-4: Iver-106 XY Trajectory summary with DVL. IMU EKF (green) and DR EKF (magenta), and CL EKF (light blue) are all DVL-aided. The reference GPS position fixes (blue) are plotted for comparison. 


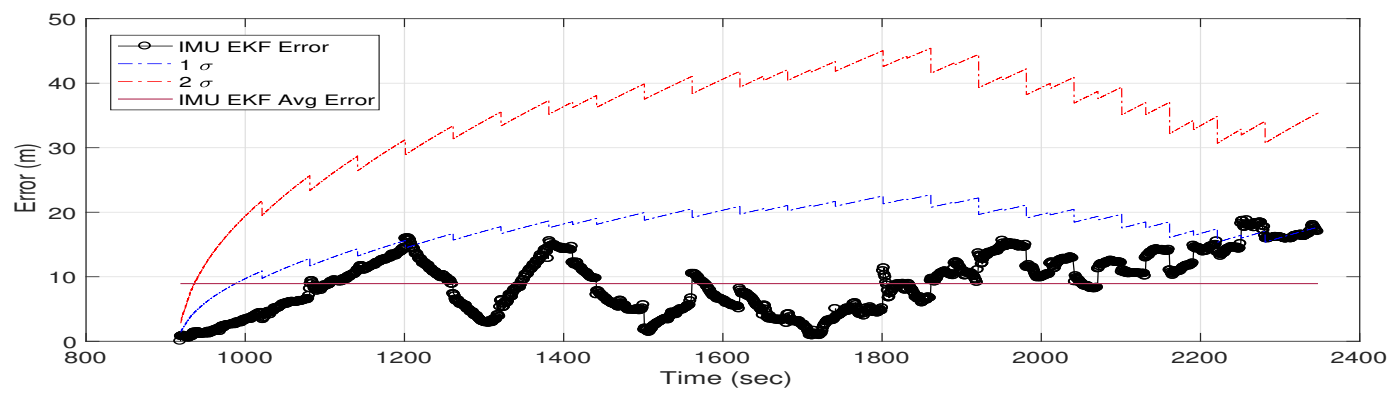

Figure 4-5: Iver-106 IMU EKF (DVL-aided) Error Summary. IMU EKF error (black) plotted with the $1 \sigma$ (blue) and $2 \sigma$ (red) uncertainty lines with the average IMU EKF error (brown). Error is the distance of the solution's estimated position from the GPS position.

\section{Bias Estimation \& Inter-vehicle Range Comparison}

Another useful analysis for the IMU EKF performance is the effect of accelerometer bias estimation and the input of OWTT range measurements from multiple beacons. In postprocessing, the IMU EKF solution was re-navigated with the different combinations of bias estimation and numbers of beacons available for OWTT range measurements. The model velocity was used for the speed measurement in all of these scenarios. All of the parameters displayed in Table 4.2 remained the same for these re-navigated solutions, except the process noise gain, $\tilde{q}$, is equal to 100 instead of 1.0 for both Iver-106 and Iver-136 for those scenarios with no bias estimation. A summary of the average error results for both Iver-106 and Iver136 is presented in Table 4.6. In this table, the "Y" indicates that the bias estimator or both beacons were used in the solution. Conversely, the "N" indicates that the bias estimator was not used or only the topside beacon was used for the OWTT range measurements.

Table 4.6: IMU EKF Beacon \& Bias Analysis Results Summary

(a) Iver-106 IMU EKF

\begin{tabular}{|c|c|c|}
\hline All Beacons & Bias Estimation & Average Error \\
\hline $\mathrm{Y}$ & $\mathrm{Y}$ & $10.26 \mathrm{~m}$ \\
\hline $\mathrm{Y}$ & $\mathrm{N}$ & $13.34 \mathrm{~m}$ \\
\hline $\mathrm{N}$ & $\mathrm{Y}$ & $14.04 \mathrm{~m}$ \\
\hline $\mathrm{N}$ & $\mathrm{N}$ & $13.86 \mathrm{~m}$ \\
\hline
\end{tabular}

(b) Iver-136 IMU EKF

\begin{tabular}{|c|c|c|}
\hline All Beacons & Bias Estimation & Average Error \\
\hline $\mathrm{Y}$ & $\mathrm{Y}$ & $12.95 \mathrm{~m}$ \\
\hline $\mathrm{Y}$ & $\mathrm{N}$ & $17.82 \mathrm{~m}$ \\
\hline $\mathrm{N}$ & $\mathrm{Y}$ & $14.26 \mathrm{~m}$ \\
\hline $\mathrm{N}$ & $\mathrm{N}$ & $17.09 \mathrm{~m}$ \\
\hline
\end{tabular}


As the results in Table 4.6 show, both the bias estimator and the use of the other vehicle serving as a beacon aided in reducing the overall position error by significant margins. For Iver-106, with both beacons present, the bias estimator included in the IMU EKF improved the solution by $23.1 \%$. Without the bias estimator, including the other vehicle as beacon improved the solution by only $3.8 \%$, which is considered insignificant and within the uncertainty of the experiment. With only the surface beacon present, the bias estimator did not improve the average error, but the average error increase was less than 1 meter, which is considered minimal. However, the largest improvement of $26.9 \%$ was made by including the additional beacon when bias estimation was present.

For Iver-136, similar results were observed. Bias estimation improved the solution by $27.3 \%$ with both beacons present. Unlike Iver-106, without bias estimation present and with the additional beacon, performance decreased, but the average error increase was less than 1 meter and is considered within the experimental uncertainty. Additionally, unlike Iver-106, with a single beacon, bias estimation lowered the error by $16.6 \%$. Nonetheless, similar to Iver-106, the addition of another beacon improved the solution by $9.2 \%$ with bias estimation present. Overall, the additional beacon had less of an influence on solution improvement than did the presence of bias estimation, but the combination of the two contributed an overall increase in solution accuracy.

Plots of the acceleration bias estimation during the course of the field trial demonstrate the positive effect of subtracting the estimated bias from other state variables. Figure 4-6 shows time series plots of the IMU EKF's estimated acceleration bias in both the $\mathrm{X}$ and $\mathrm{Y}$ world frame dimensions. As the plot shows, the estimated bias (red) is plotted on top of the raw world frame IMU acceleration measurements, which helps to show that the GaussMarkov model for acceleration bias processed through the IMU EKF tracks over time with the bias exhibited by the raw IMU acceleration measurements. Table 4.7 shows a summary of the significant improvement for both Iver-106 and Iver-136 in reducing the average error with use of the bias estimator for both one and two beacons. As this table shows, there is improvement in all categories except for Iver-106 when only the surface beacon was used, which was a minimal decline. Because of these results, analysis of the subsequent field trials all include bias estimation and multiple beacons where available. 

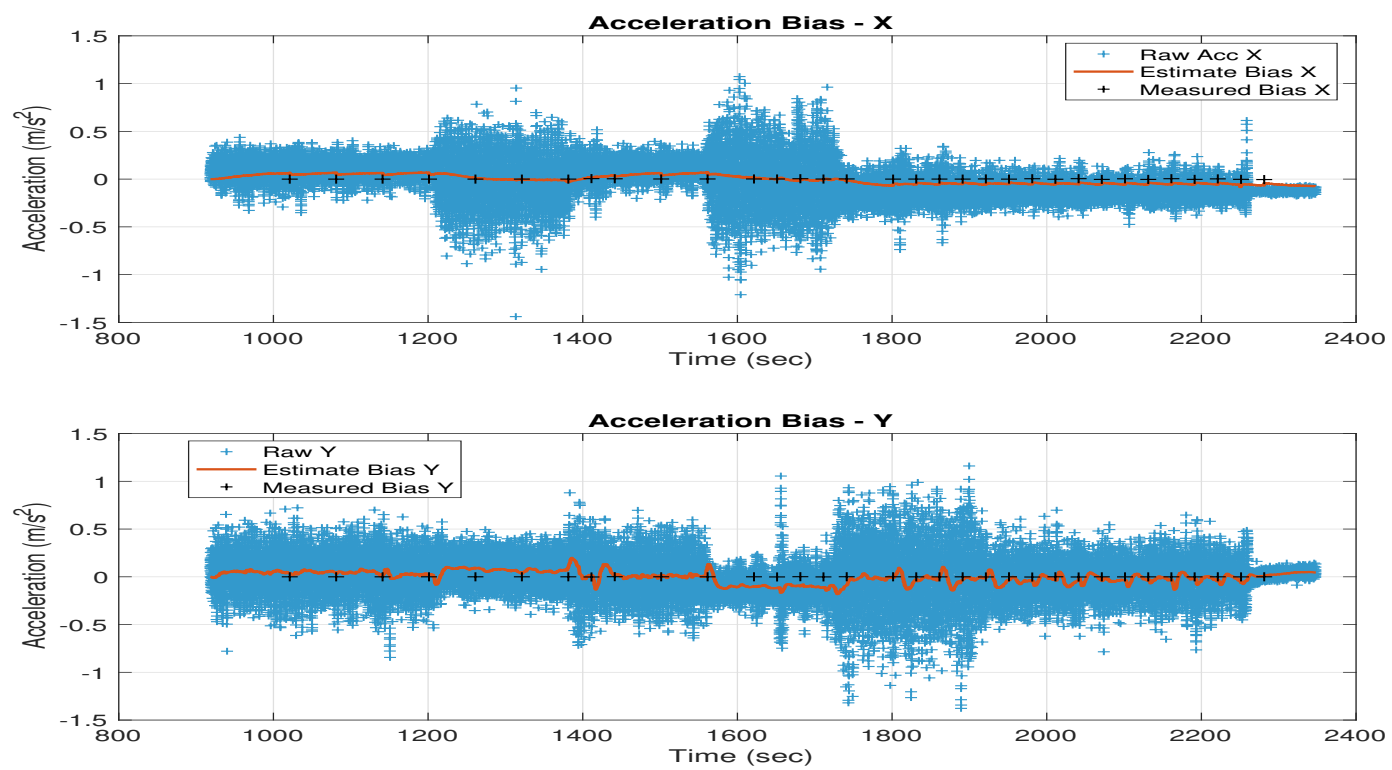

(a) Iver-106 Bias Estimation
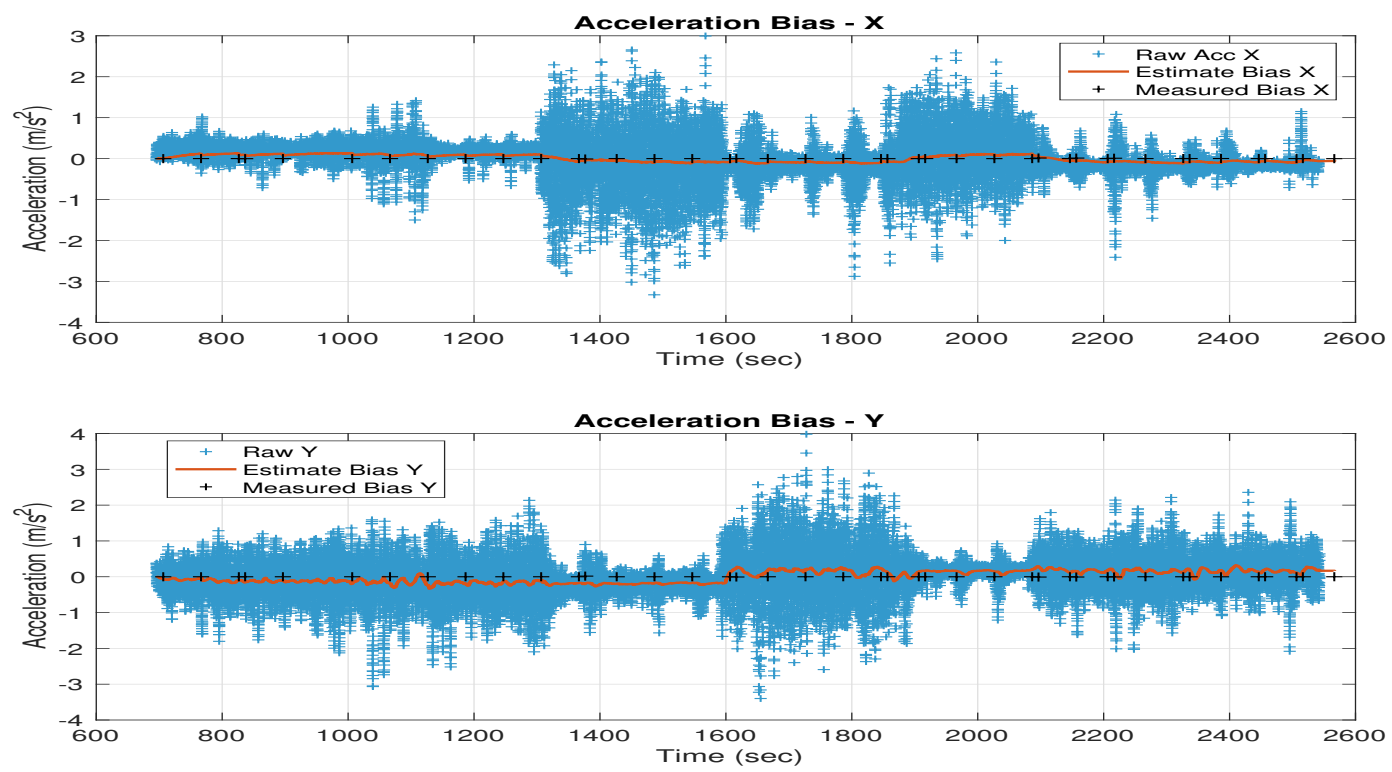

(b) Iver-136 Bias Estimation

Figure 4-6: IMU EKF Bias Estimation Results. [Top] Iver-106 bias estimation results. [Bottom] Iver-136 bias estimation results. Raw acceleration measurements in the world frame (cyan), acceleration bias measurements in the world frame (block crosses), and IMU EKF bias estimate in world frame (red) are plotted together for comparison.

\subsection{Charles River Trials - October 2016}

The Bluefin Robotics, Inc. SandShark $\mu$ AUV was deployed for a field trial on the Charles River on October 17, 2016 for the Massachusetts Institute of Technology (MIT) Laboratory for Acoustic Marine Sensing Systems. The main purpose of this field trial was to assess 
Table 4.7: IMU EKF Performance Improvement Results with Bias Estimation

(a) Iver-106 IMU EKF

\begin{tabular}{|c|c|}
\hline All Beacons & Solution Improvement \\
\hline $\mathrm{Y}$ & $23.1 \%$ \\
\hline $\mathrm{N}$ & $-1.3 \%$ \\
\hline
\end{tabular}

(b) Iver-136 IMU EKF

\begin{tabular}{|c|c|}
\hline All Beacons & Solution Improvement \\
\hline $\mathrm{Y}$ & $27.3 \%$ \\
\hline $\mathrm{N}$ & $16.6 \%$ \\
\hline
\end{tabular}

the accuracy of the IMU EKF on a different platform, acoustic environment, and mission trajectory. Additionally, this experiment proved the the importance and necessity of the coupled range filter to the IMU EKF to preserve solution accuracy.

The vehicle's closed-loop navigation solution that was used during this field trial was a model velocity based dead-reckoned solution (referred to as SS CL); it used GPS measurements but did not incorporate any OWTT range measurements. Both the IMU EKF and DR EKF implementations on this field data used a model velocity for speed measurements, but neither of these solutions used GPS. The IMU EKF did process the OWTT measurements from the topside beacon, but the DR EKF did not process these measurements nor did it incorporate any bias estimation.

The IMU EKF implemented herein on the SandShark is the same as presented in the prior section for the Iver2 AUVs except that this IMU EKF processed a median range from the four ranges acquired by the tetrahedral array described in Section 3.1.2. The parameters for the SandShark IMU EKF are summarized in Table 4.8.

Table 4.8: SandShark IMU EKF Parameter Values

\begin{tabular}{|c|c|}
\hline Parameter & Value \\
\hline$d t$ & $0.015 \mathrm{~s}$ \\
\hline$\sigma_{b}$ & $1.0 \mathrm{~m} / \mathrm{s}^{2}$ \\
\hline$T_{c_{x}}$ & $1000 \mathrm{~s}$ \\
\hline$T_{c_{y}}$ & $1000 \mathrm{~s}$ \\
\hline$\tilde{q}$ & 0.1 \\
\hline Range Threshold & $130 \mathrm{~m}$ \\
\hline Max Speed & $1.54 \mathrm{~m} / \mathrm{s}$ \\
\hline$\sigma_{\text {rng }}$ & $3.3 \mathrm{~m}$ \\
\hline$\sigma_{\text {vel }}$ & $1.0 \mathrm{~m} / \mathrm{s}$ \\
\hline$\sigma_{\text {accel }}$ & $0.1 \mathrm{~m} / \mathrm{s}^{2}$ \\
\hline$\sigma_{\text {bias }}$ & $0.1 \mathrm{~m} / \mathrm{s}_{2}$ \\
\hline
\end{tabular}




\subsubsection{Trajectory Summary}

The SandShark $\mu \mathrm{AUV}$ was deployed from the sailing center dock at the MIT Sailing Pavilion on the Charles River. The vehicle's navigation solution was initialized on the dock, hand carried to the water, then deployed for its mission and immediately submerged. The vehicle followed a lawnmower pattern trajectory with a mission time of 15.85 minutes, submerged at a depth of 2 meters with a commanded speed of $1 \mathrm{~m} / \mathrm{s}$ except when on the surface. The vehicle surfaced twice during the mission: once at approximately nine minutes into the mission and then at mission completion. The topside beacon transmitted acoustic packets at a frequency of $1 \mathrm{~Hz}$, and the vehicle experienced $28.50 \%$ acoustic packet loss over the course of its mission.

\subsubsection{Performance Analysis}

Due to the high frequency of range measurements from the topside beacon at $1 \mathrm{~Hz}$, the highly reflective acoustic environment of the Charles River, and effect of self-occlusion on the matched filtering process [40], many grossly inaccurate ranges were observed by the tetrahedral array on the vehicle. Figure 4-7 shows the numerous erroneous ranges, both those that were longer and shorter than the actual range of the vehicle from the beacon.

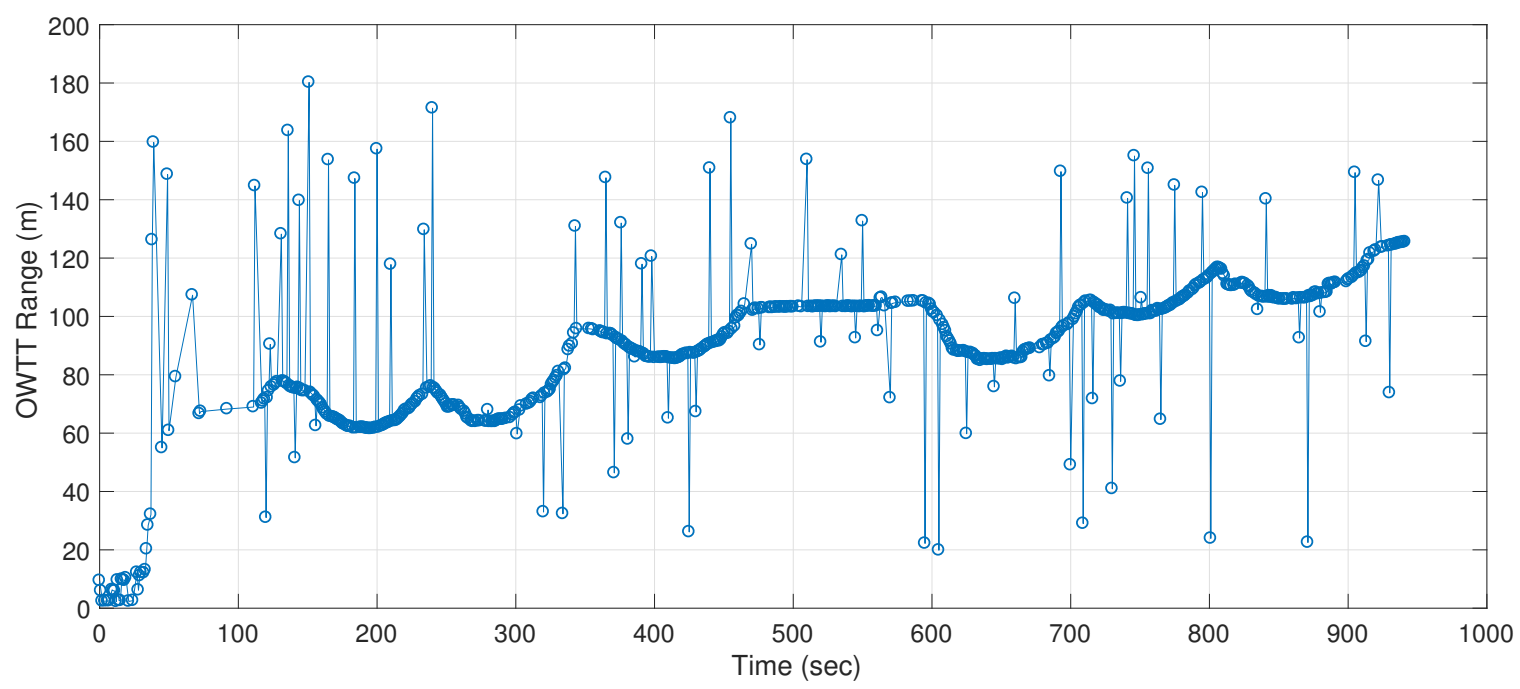

Figure 4-7: SandShark $\mu$ AUV received raw median OWTT ranges. This plot shows the variability in the OWTT range measurements due to the highly reflective acoustic environment of the Charles River.

Without using the coupled range filter, the IMU EKF resulted in an unstable trajectory (Figure 4-8) by processing of all of the raw OWTT ranges. However, by including the 
coupled range filter with the parameter values shown in Table 4.8, significant improvement was observed in the vehicle's XY trajectory. Of the 686 OWTT raw ranges from the topside beacon, 26 ranges were removed by the range-threshold filter, and 62 ranges were removed by the state acceptance gate. Figure 4-9 shows the IMU EKF processed OWTT ranges against the received raw OWTT ranges. As this figures shows, the coupled range filter prevented a majority of the erroneous ranges from being processed by the IMU EKF.

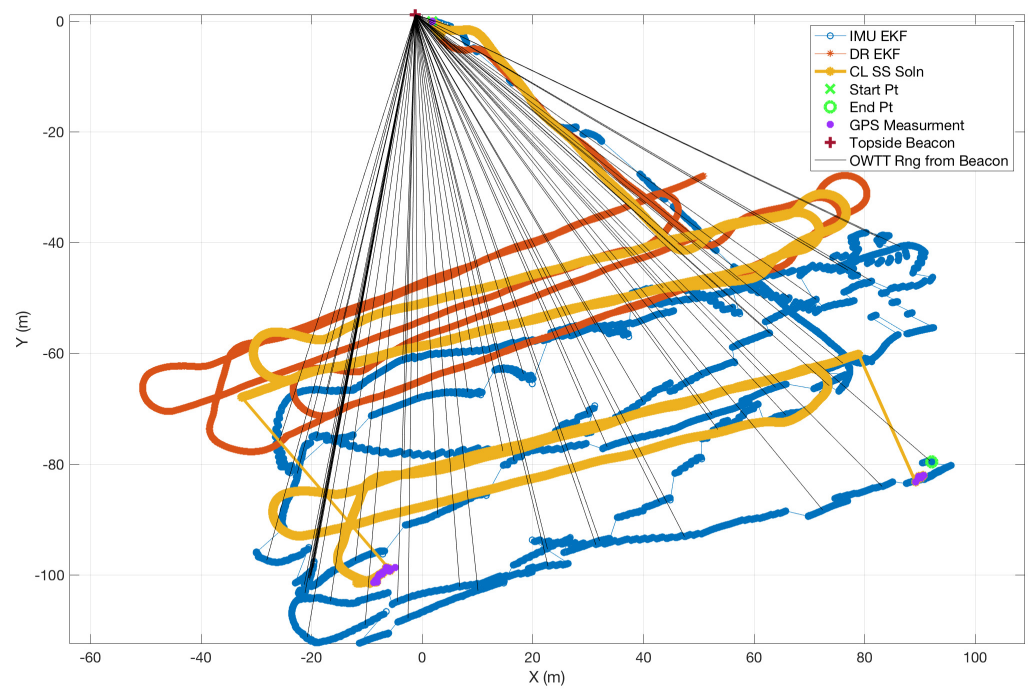

Figure 4-8: SandShark Position summary without the coupled range filter. SandShark IMU EFK trajectory (blue) without the coupled range filter is unstable. The DR EKF (red) and the SS CL Solution (gold) along with the GPS points (dark blue) are plotted for reference and comparison. The black lines represent the OWTT ranges from the stationary topside beacon to the updated position state of the vehicle. Every 10th OWTT range is plotted to prevent plot clutter.

By processing the OWTT ranges given by the coupled range filter as shown in Figure 4-9, a more stable trajectory resulted as shown in Figure 4-10, which shows the IMU EKF (blue), the DR EKF (red), and the CL SS (gold) navigation solution. As the plot illustrates, the IMU EKF is the solution that most closely matches the GPS measurements when the vehicle surfaces at the mission's midpoint and endpoint.

The source of ground truth for this field data was the GPS position fixes during the two surfacing periods in the mission. Figure 4-11 displays the time series error of the three navigation solutions compared to GPS position, and the IMU EKF exhibited an average error of 12.23 meters, which was the smallest error of all three solutions for the SandShark. 


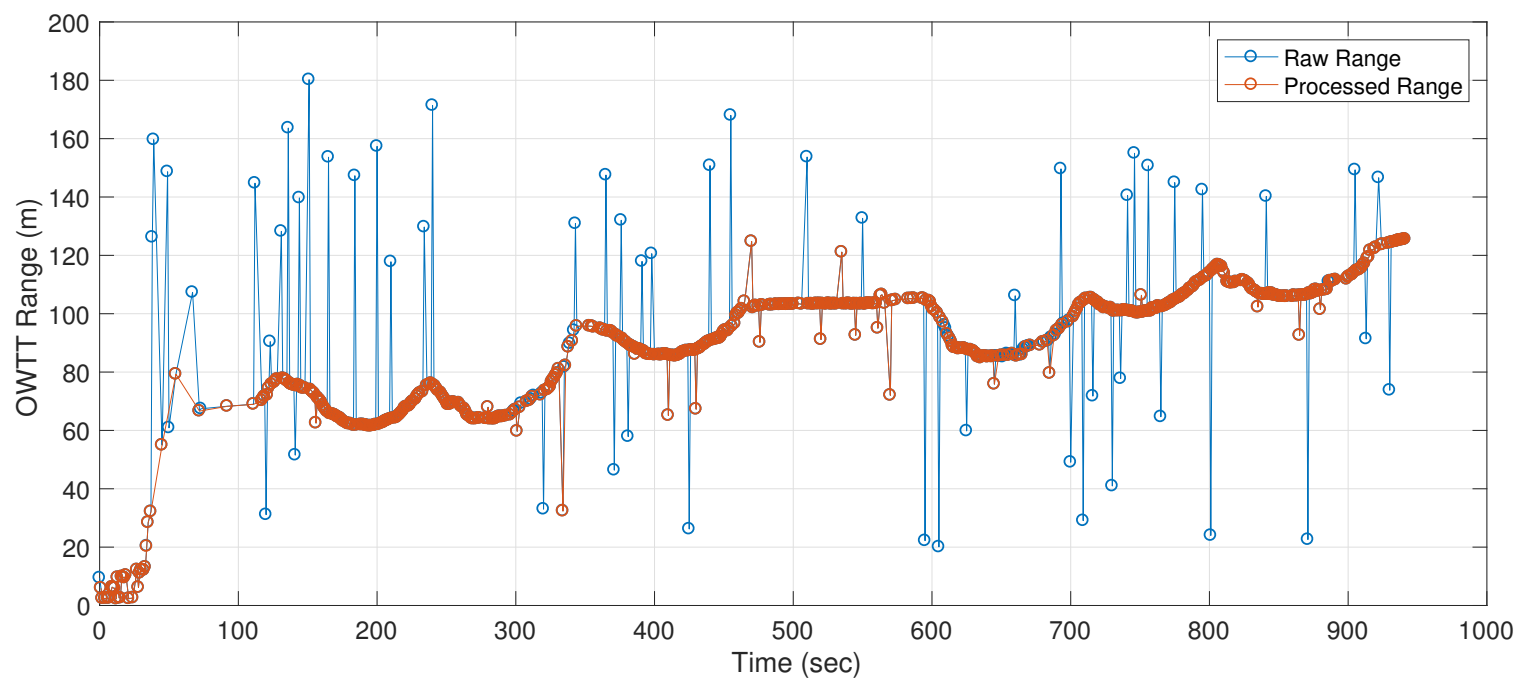

Figure 4-9: SandShark Processed OWTT Ranges. The raw OWTT ranges (blue) are plotted with the processed OWTT ranges (red) by the IMU EKF. These processed ranges are a result from passing the required conditions of the coupled range filter.

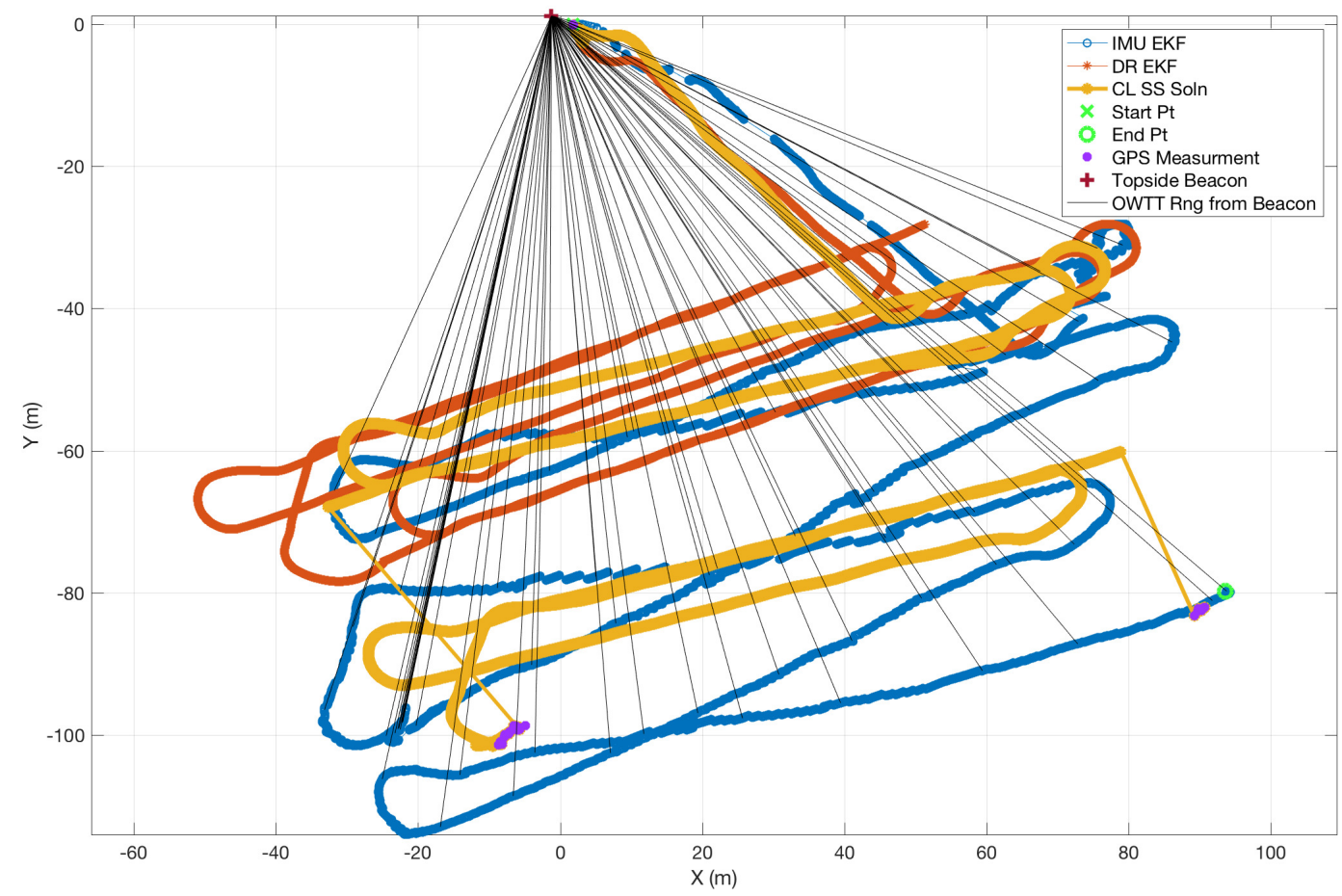

Figure 4-10: SandShark EKF Position Summary: IMU EKF (blue), DR EKF (red), Sand Shark CL solution (gold). The black lines indicate the OWTT ranges from the stationary topside beacon to the vehicle's updated position. To prevent plot clutter, every 10th range processed by the IMU EKF is plotted. 
Average errors from GPS positions for the other solutions are summarized in Table 4.9. In order to provide a more accurate comparison, points after the SS CL solution accepted GPS position fixes are removed from the error calculation for the average value shown in the table.

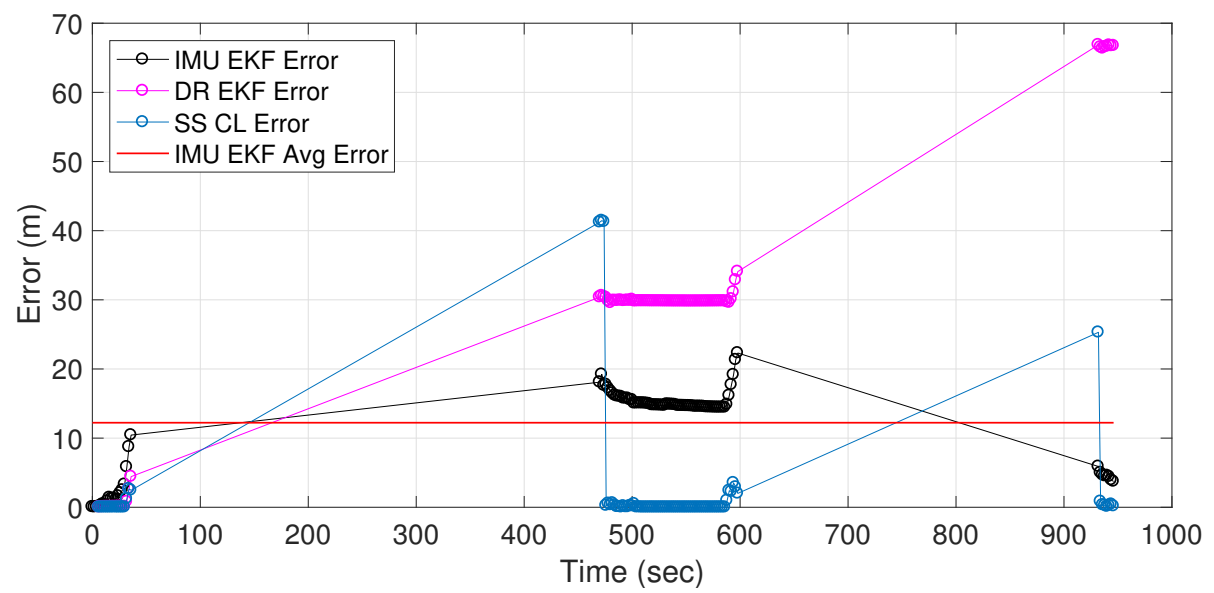

Figure 4-11: SandShark Performance Summary. This plot displays the IMU EKF error (black), the DR EKF error (magenta), the SandShark CL DR error (light blue) compared to the GPS locations at the time the vehicles surfaced during the mission. The average IMU EKF error (brown) is plotted for comparison. The SS CL solution exhibits the minimum average error because it processed GPS twice during the mission. This metric is misleading because the SS CL solution accrued the most error before the first surfacing with approximately 40 meters of error and accrued approximately 25 meters of error before its second surfacing. The IMU EKF accrued less error than the SS CL solution during both of these intervals.

Table 4.9: SandShark Navigation Performance Summary

\begin{tabular}{|c|c|}
\hline Parameter & Performance Result \\
\hline Amount of Filtered Ranges & $12.94 \%$ \\
\hline IMU EKF Average Error & $12.22 \mathrm{~m}$ \\
\hline DR EK Average Error & $27.99 \mathrm{~m}$ \\
\hline SS CL Average Error & $37.32 \mathrm{~m}$ \\
\hline
\end{tabular}

\subsection{Ashumet Pond Trials - April 2017}

A third field trial was conducted on Ashumet Pond on April 20, 2017 to provide additional field data for a real-time implementation of the IMU EKF. The main purpose of this field trial was to assess the reliability of the IMU EKF with the coupled range filter in a multiple 
vehicle mission trajectory that resembled current, real-world AUV applications. Thus, this experiment consisted of submerged legs that were longer in length and time than those of the previous trials.

\subsubsection{Trajectory Summary}

A dual-vehicle, lawn-mower type trajectory was administered for these field trials, using the same vehicles with the same configurations and ACOMMS network as those used in the October 2016 experiments. Both Iver-106 and Iver-136 used their constant-velocity model EKF (i.e., CL EKF), externally aided by TOF range measurements from a surface beacon as well as by GPS. Both vehicles were planned to run orthogonal submerged legs in order to maximize position constraints from the TOF range measurements from each other and the surface beacon. The planned mission for each vehicle is displayed in Figure 4-12. Periodic

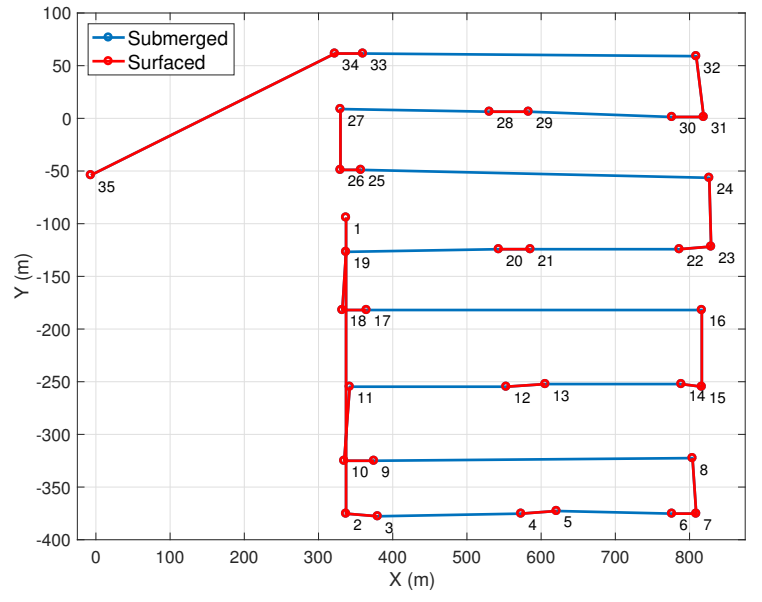

(a) Iver106 Mission

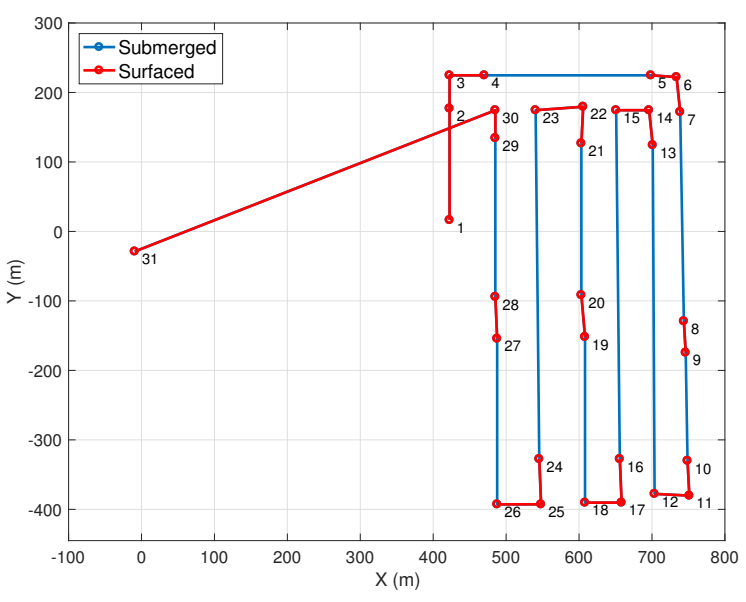

(b) Iver136 Mission

Figure 4-12: AUV Planned Mission Trajectory. Iver-106 [Left] and Iver-136 [Right] planned mission trajectories. Each vehicle was planned to run orthogonal submerged legs (blue) with periodic surfacings (red) throughout the mission. Each point contains its respective waypoint number.

surfacings were planned through out the course of the mission to provide periodic position fixes from GPS. These GPS points served as the position's truth source for later analysis. Each submerged leg was conducted at depth of 3 meters for a length of approximately 250 meters or approximately 500 meters. Commanded speed for both vehicles throughout the entire mission was 2 knots $(1.03 \mathrm{~m} / \mathrm{s})$. The total planned mission length was approximately 5 kilometers for each vehicle with a mission time of approximately 1.5 hours. Iver-136 
was deployed first, shortly followed by Iver-106. Due to various difficulties with Iver-106 after first being deployed, the mission was stopped and recommenced after troubleshooting, thus the presented Iver-106 mission does not start until about 2000 seconds after Iver-136. However, during the first 2000 seconds of the Iver-136 mission, the vehicle did receive ranges from Iver-106 during the troubleshooting process. The surface skiff, which provided the surface beacon with access to GPS, drifted in the area for the entirety of the mission.

\subsubsection{EKF Range Sensitivity Discussion}

This field data provides great opportunity to assess the functionality and the importance of the coupled range filter discussed in Section 2.2.3. As discussed prior, the navigation solution used on the vehicles at the time of this data collection was a constant-velocity model EKF (referred to as the CL EKF). However, this CL EKF did not incorporate any additional filtering on the TOF range measurements, so it was susceptible to faulty ranges due to poor clock synchronization, acoustic environment, or poor position state estimates provided by the transmitting beacon. Figure 4-13 shows the CL EKF trajectory summaries for both Iver-106 and Iver-136. These two plots clearly demonstrate how the absence of additional range filtering can adversely affect the accuracy of the navigation solution. Further, Figure 4-14 shows a time series plot of the different OWTT range measurements received and processed by the two vehicles from both beacons, and this plot shows the distinct OWTT ranges and the clear unreliable measurements due to poor clock synchronization or a variable acoustic environment. For Iver-106, one such range occurred at approximately 4700 seconds, and Iver-136 observed two such faulty ranges in the beginning of the mission and at 1000 seconds.

\section{EKF Narrative}

The following narrative is an explanation of the odd behavior exhibited by both Iver-106 and Iver-136 during these field trials because of the CL EKF processing faulty range measurements. A detailed understanding of these events is necessary since the data collected during this field trial (which includes the odd vehicle behavior therein) was used to implement the IMU EKF (further discussed in section 4.3.3). All of the events are referenced to mission time, $\mathrm{t}$, as shown in the previous plots.

$\underline{t=0 \text { sec: }}$ Both vehicles' mission commenced. 


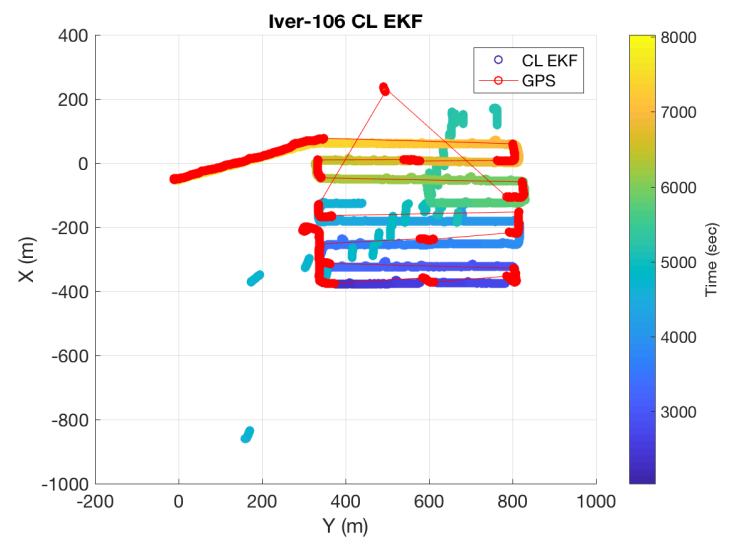

(a) Iver106 CL EKF Trajectory

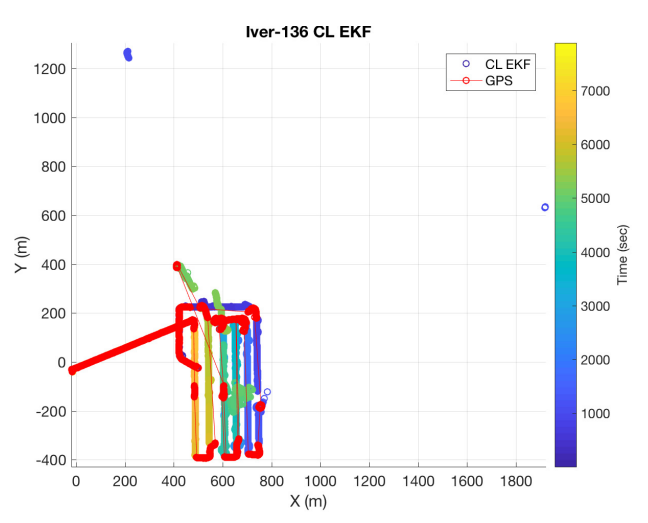

(b) Iver136 CL EKF Trajectory

Figure 4-13: Closed-loop EKF Trajectory Summaries. [Left] Iver-106 CL EKF trajectory summary, and [Right] Iver-136 CL EKF trajectory. Each summary shows a time color bar plot of the vehicle's estimated position along with its GPS position fixes (red) when the vehicle surfaced. Iver-136 was deployed first, and Iver-106 was deployed approximately 2000 seconds after Iver-136. These plots show the significant effect of not applying additional filtering on the TOF range measurements. Note the difference in scale between the two plots.

$\underline{t=647-2000 \text { sec: }}$ Observed odd behavior on Iver-106. Iver-106 mission stopped to investigate and correct cause with vehicle on the surface. During various periods in this time window, Iver-106 navigation solution transmitted its estimated position to Iver-136.

$\underline{t=167 \mathrm{sec}}:$ Iver-136 received faulty range ( 2100 meters) from Iver-106 (Figure 4-14b). Iver-136 CL EKF was not affected due to receipt of GPS while transiting between waypoints 3 and 4 (Figure 4-12b).

$\underline{t=1060 \text { sec: }}$ Iver-136 received faulty range ( 2065 meters) (Figure 4-14b) from topside beacon, causing the CL EKF position to jump to the northeast to position $(1919,630)$ (Figure $4-13 b)$.

$\underline{t=1067 \text { sec: }}$ Iver-136 received range from Iver-106, which shifted CL EKF position to the north to position $(213,1271)$ (Figure 4-13b).

$\underline{t=1098 \text { sec: }}$ Iver-136 received GPS position fix, CL EKF reconverged, and continued to proceed between waypoints 9 and 10 (Figure 4-12b).

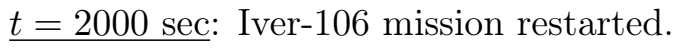

$\underline{t=3171 \mathrm{sec}}$ : Iver-136 retasked to repeat portion of mission to lengthen mission time since Iver-106 was delayed in mission start. Iver-136 retasked to repeat mission starting at waypoint 15 (Figure 4-12b). 


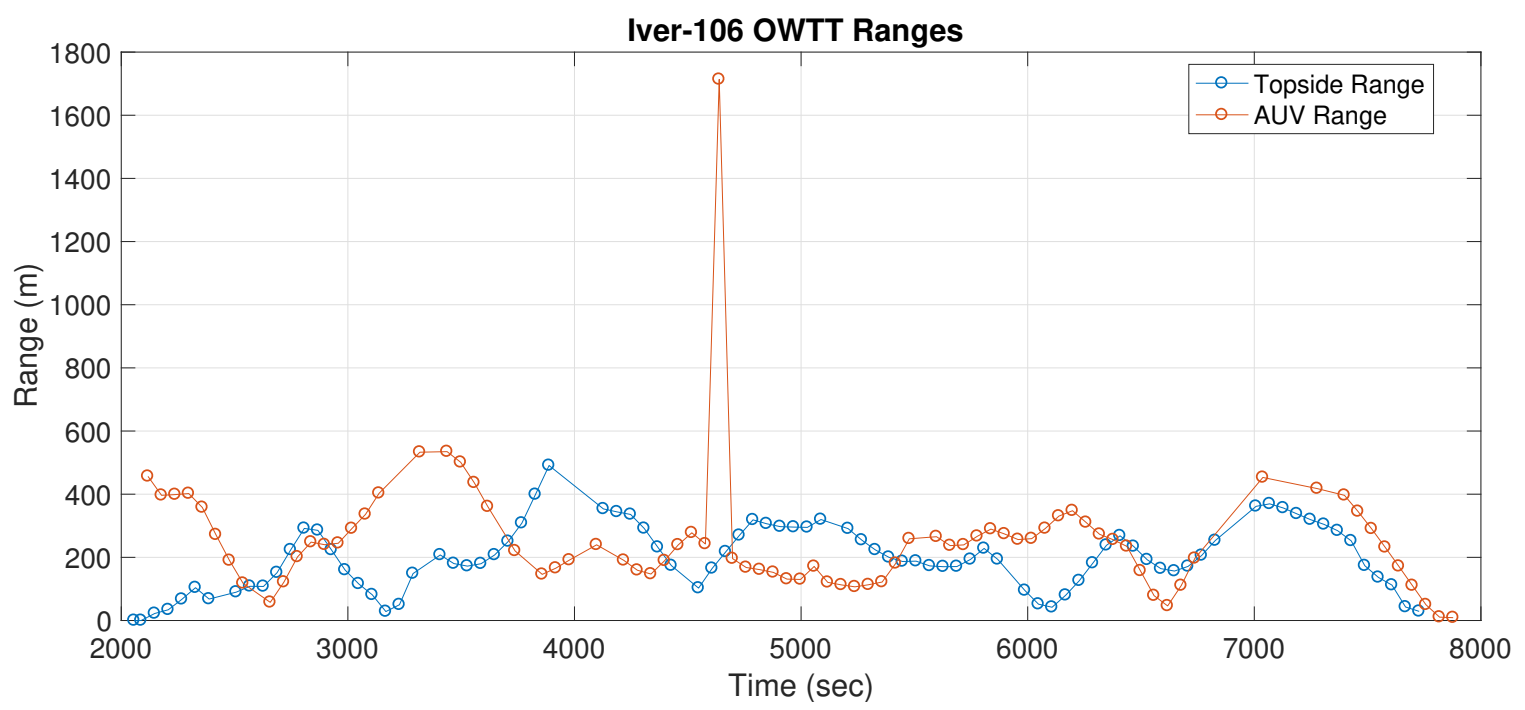

(a) Iver106 CL EKF Range Measurement Summary

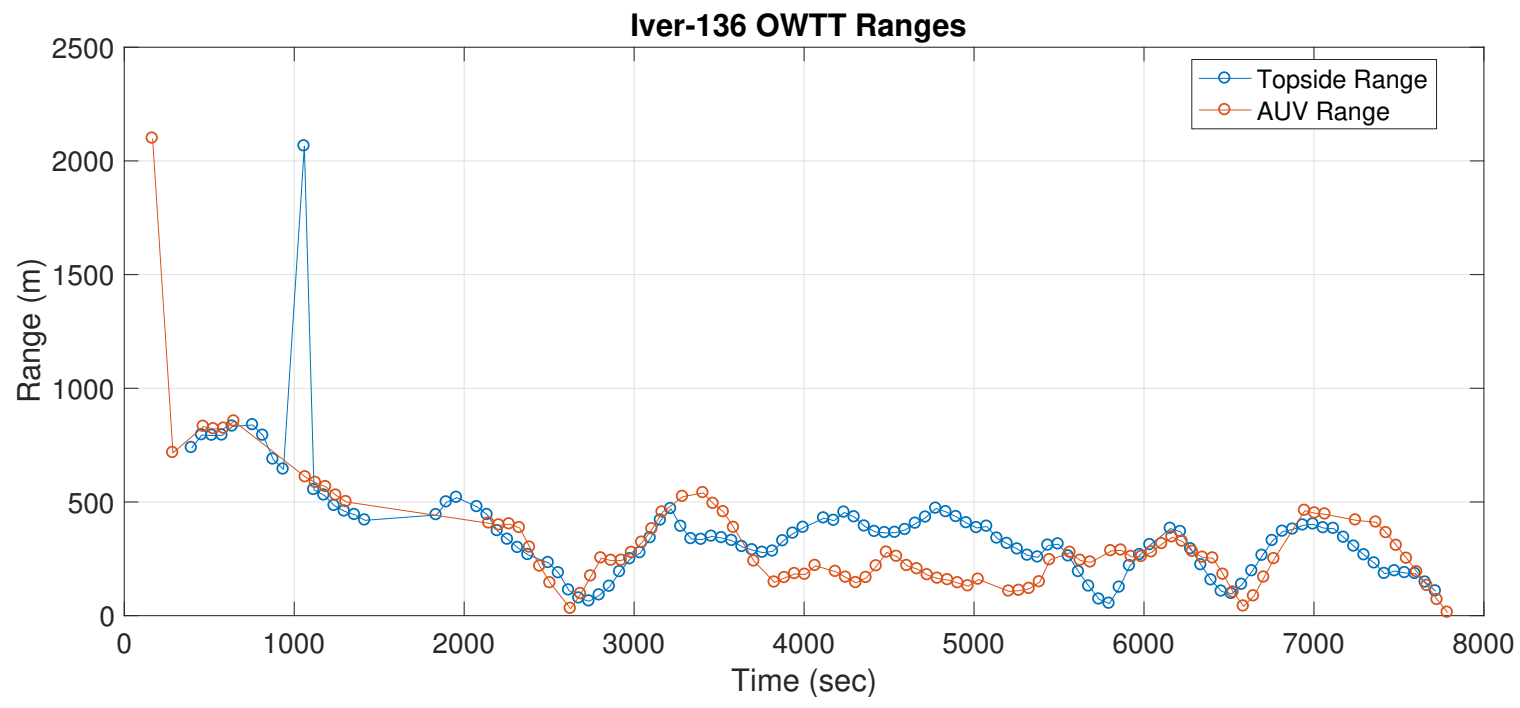

(b) Iver136 CL EKF Range Measurements Summary

Figure 4-14: Closed-loop EKF Range Measurement Time Series Summaries. Iver-106 [Top] and Iver-136 [Bottom] CL EKF OWTT range measurements. Both vehicles received and processed OWTT range measurements from the topside beacon (blue) and from the other vehicle (red).

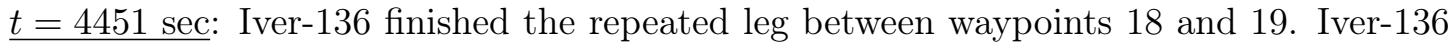
completed surfacing of the repeated leg between waypoints 19-20 and resubmerged (Figure $4-12 b)$.

$\underline{t=4639 \mathrm{sec}}:$ Iver-106 received faulty range $(\sim 1713$ meters) from Iver-136 (Figure 414a), causing Iver-106 CL EKF to offset position to southwest to position (158,-860) (Figure 4-13a). Iver-106 started to travel north to regain track. 
$\underline{t=4666 \text { sec: }}$ Iver-136 received range from Iver-106 when Iver-106 had faulty state estimates (period between 4639 - 5300 seconds), which caused Iver-136 to offset to the east and caused Iver-136 to drive northwest to regain track. Iver-136 received multiple other ranges from both the topside beacon and Iver-106 while trying to regain track by driving northwest.

$\underline{t=4977 \text { sec: }}$ Iver-106 surfaced to the north at position $(490,210)$ (Figure 4-13a) but did not process the GPS fix due to improper zeroing of its depth sensor. Not processing the GPS fix at this time delayed the vehicle's position estimate in reconverging and prolonged the instability in the navigation solution. Further range updates from Iver-136 and the topside beacon facilitated Iver-106 to travel south to regain track. At $t=\sim 5300 \mathrm{sec}$, Iver-106 reconverged on track and proceeded easterly between waypoints 21 and 22 (Figure 4-12a).

$\underline{t=5045 \mathrm{sec}}:$ Iver-136 surfaced to the northwest at position $(400,400)$ (Figure $4-13 \mathrm{~b})$ and received GPS position fix.

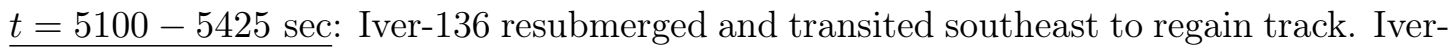
136 received multiple ranges from both the topside beacon and from Iver-106.

$\underline{t=5426 \text { sec: }}$ Iver-136 received GPS fix at waypoint 23 and started southerly leg between waypoints 23 and 24. Both Iver-106 and Iver-136 CL EKFs remained stable for remainder of the missions.

\subsubsection{Performance Analysis}

Similar to the field trials conducted in October 2016, the IMU EKF, the CL EKF, and the DR EKF are presented for analysis. For Iver-106, the CL EKF used its DVL for speed measurements as well as GPS. However, due to the vehicle's depth sensor not properly zeroing itself when on the surface, GPS fixes were not incorporated into the CL EKF starting at $t=3313$ seconds. At this point in the mission, the depth sensor indicated a depth of 0.33 meters and continued a linear degradation of approximately 0.03 meters per minute for the remainder of the mission. Iver-136, on the other hand, used a vehicle model velocity for its CL EKF's speed measurement and incorporated all GPS fixes throughout its mission. The following analysis presents each vehicle separately with a concentration on the effect of its coupled range filter. 


\section{Iver-106 Performance Discussion}

In this analysis, the IMU EKF was implemented both with the vehicle's model velocity and its DVL as the speed measurement. Additionally, due to battery replacement on Iver-106, the previous calibration for the Pololu IMU magnetometer was inaccurate, thus the manufacturer MEMS compass provided the attitude measurements. GPS was not incorporated into any of the analyses of the IMU EKF nor the DR EKF. Due to the incorporation of the coupled range filter and the manufacturer compass in this implementation, the velocity standard deviation, $\sigma_{v e l}$, and the frequency of the IMU EKF, $d t$, for the IMU EKF required different values. The parameters presented in Table 4.2 remained the same except those identified in Table 4.10. As depicted in Table 4.10, the maximum speed value for the state acceptance gate are different between the model velocity-aided and DVL-aided IMU EKFs. Since the commanded speed for the mission was $1.03 \mathrm{~m} / \mathrm{s}$, the maximum speed set at 1.29 $\mathrm{m} / \mathrm{s}$ gives a $25 \%$ conservative boundary for inaccurate model velocity speed measurements. For the DVL-aided IMU EKF, $2.06 \mathrm{~m} / \mathrm{s}$ was used because the DVL provides a more accurate speed measurement with a higher variance, and $2.06 \mathrm{~m} / \mathrm{s}$ is the maximum rated speed of the vehicle.

Table 4.10: Iver-106 IMU EKF Parameter Values

(a) Model Velocity-Aided

\begin{tabular}{|c|c|}
\hline Parameter & Value \\
\hline$\sigma_{\text {vel }}$ & $10.0 \mathrm{~m} / \mathrm{s}$ \\
\hline$d t$ & $0.085 \mathrm{~s}$ \\
\hline Max Speed & $1.29 \mathrm{~m} / \mathrm{s}$ \\
\hline
\end{tabular}

(b) DVL-Aided

\begin{tabular}{|c|c|}
\hline Parameter & Value \\
\hline$\sigma_{\text {vel }}$ & $5.0 \mathrm{~m} / \mathrm{s}$ \\
\hline$d t$ & $0.085 \mathrm{~s}$ \\
\hline Max Speed & $2.06 \mathrm{~m} / \mathrm{s}$ \\
\hline
\end{tabular}

The trajectory summaries for the IMU EKF using both the vehicle model velocity and the DVL are shown in Figure 4-15, and the error summaries are shown in Figure 4-16. As the plots show, the IMU EKF tracks closely with the GPS measurements throughout the mission for both types of speed measurements. Both types of solutions are comparable in accuracy, but, as expected, the DVL-aided IMU EKF lowered the position uncertainty by approximately a factor of two. These IMU EKF solutions do not exhibit the same erratic behavior of the CL EKF, and both of these solutions show true vehicle movement to the North as evident by the GPS fix at position $(490,225)$ on Figure 4-15. With this more accurate trajectory, both of the IMU EKF errors are considerably less than the CL EKF 
error, especially at time, $\mathrm{t}=5000$ seconds when the vehicle drove north, as shown in Figure $4-17$.

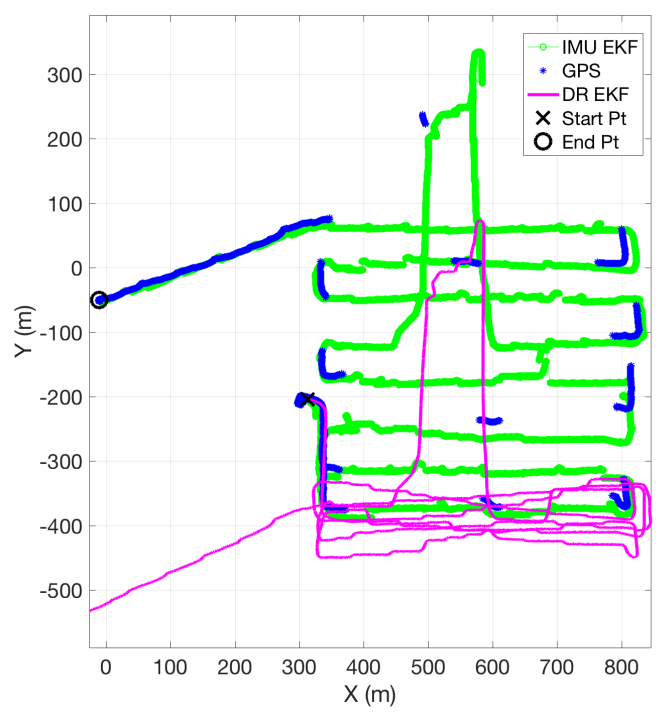

(a) Iver-106 IMU EKF Trajectory - Model Velocity

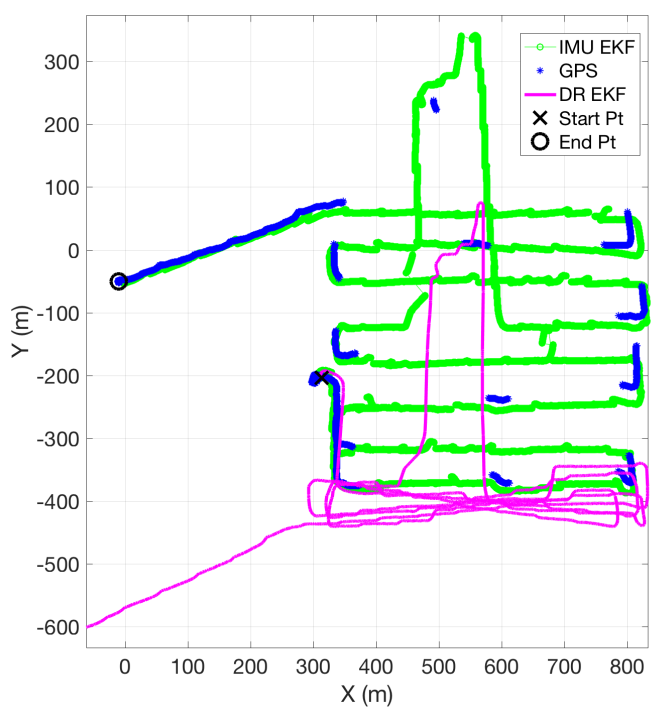

(b) Iver-106 IMU EKF Trajectory - DVL

Figure 4-15: Iver-106 IMU EKF Trajectory Plot. The IMU EKF solution (green) and the DR EKF (magenta) plotted against the associated GPS position fixes (blue) for comparison. [Left] IMU EKF and DR EKF with the vehicle dynamic model velocity used as its speed measurement. [Right] IMU EKF and DR EKF with the DVL used as the speed measurement.

Table 4.11: Iver-106 EKF Performance Summary

\begin{tabular}{|c|c|c|}
\hline Solution & DVL-Aided & Model Velocity-Aided \\
\hline IMU EKF & $11.93 \mathrm{~m}$ & $12.32 \mathrm{~m}$ \\
\hline CL EKF & $12.87 \mathrm{~m}$ & $\mathrm{~N} / \mathrm{A}$ \\
\hline DR EKF & $283.89 \mathrm{~m}$ & $257.45 \mathrm{~m}$ \\
\hline Distance Traveled & $5.28 \mathrm{~km}$ & $5.28 \mathrm{~km}$ \\
\hline
\end{tabular}

Table 4.11 displays the summary of the average errors of all the EKF solutions, and Figure 4-17 shows the time series of all the EKF solution errors. As shown, the model velocity-aided IMU EKF is competitive with both DVL-aided IMU EKF and CL EKF solutions. Unexpectedly, the DVL-aided DR EKF performed worse than the model velocityaided DR EKF for unknown reasons. This discrepancy was not observed in any other experiment, and it does not undermine the performance of the IMU EKF solutions. 


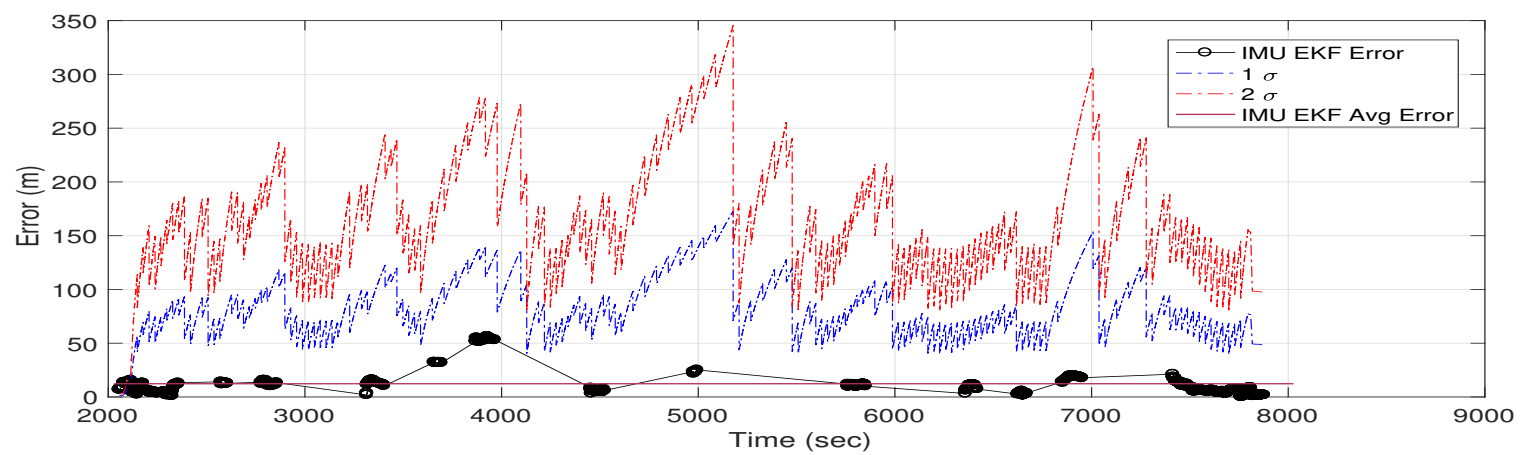

(a) Iver-106 IMU EKF Error - Model Velocity

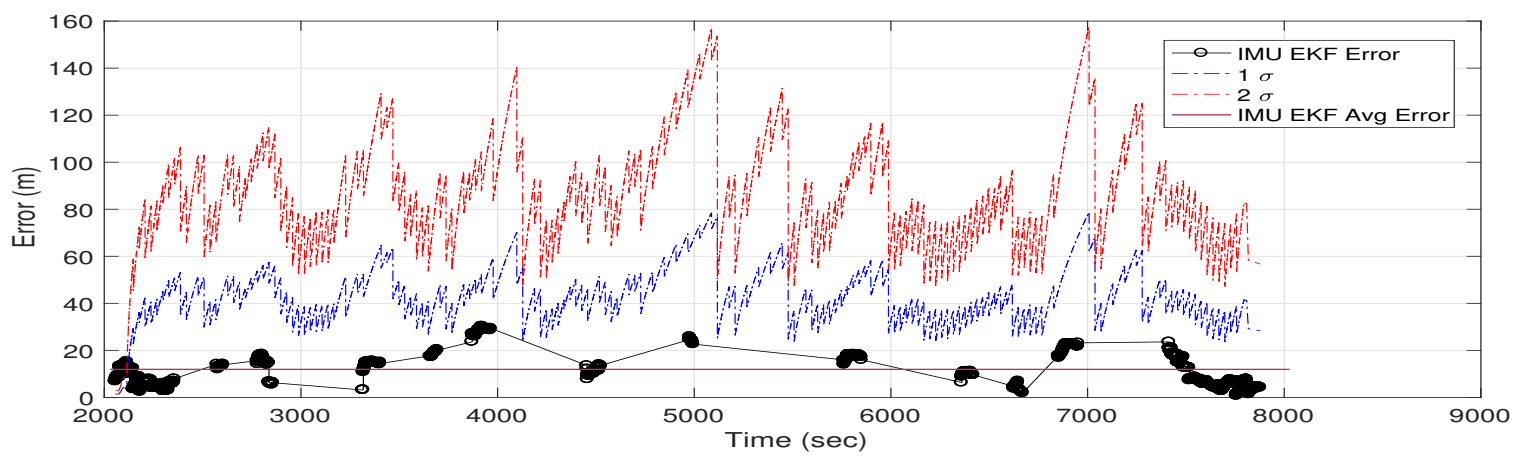

(b) Iver-106 IMU EKF Error - DVL

Figure 4-16: Iver-106 IMU EKF Error Summary: [Top] IMU EKF error with model velocity. [Bottom] IMU EKF error with DVL. These plots contain the IMU EKF error (black), its average error (brown), and its associated $1 \sigma$ (blue) and $2 \sigma$ (red) uncertainty lines. Note the difference in scales between the two plots, thus showing the DVL-aided solution provided a more accurate estimate.

\section{Iver-136 Performance Discussion}

Contrasting to the field trials conducted during October 2016, the DVL on Iver-136 was operational. Although the model velocity was infused into the CL EKF, the DVL remained available for the IMU EKF. Thus, the implementation of the IMU-EKF on this field data for Iver-136 used both a model velocity and its DVL. All of the parameter values for the IMU EKF presented in Table 4.2 remain the same for this implementation, except those identified in Table 4.12. Experimental speed trials identified that the vehicle reaches a maximum speed of $1.54 \mathrm{~m} / \mathrm{s}$ instead of its designed rated speed of $2.06 \mathrm{~m} / \mathrm{s}$. Thus, a maximum speed value of $1.54 \mathrm{~m} / \mathrm{s}$ is used for the coupled range filter. Like Iver-106, GPS was not observed for any of the IMU EKF or DR EKF implementations presented in this analysis.

The trajectory summaries for the Iver-136 IMU EKF, using both the model velocity and the DVL, are displayed in Figure 4-18, and the error summaries are shown in Figure 4-19. 


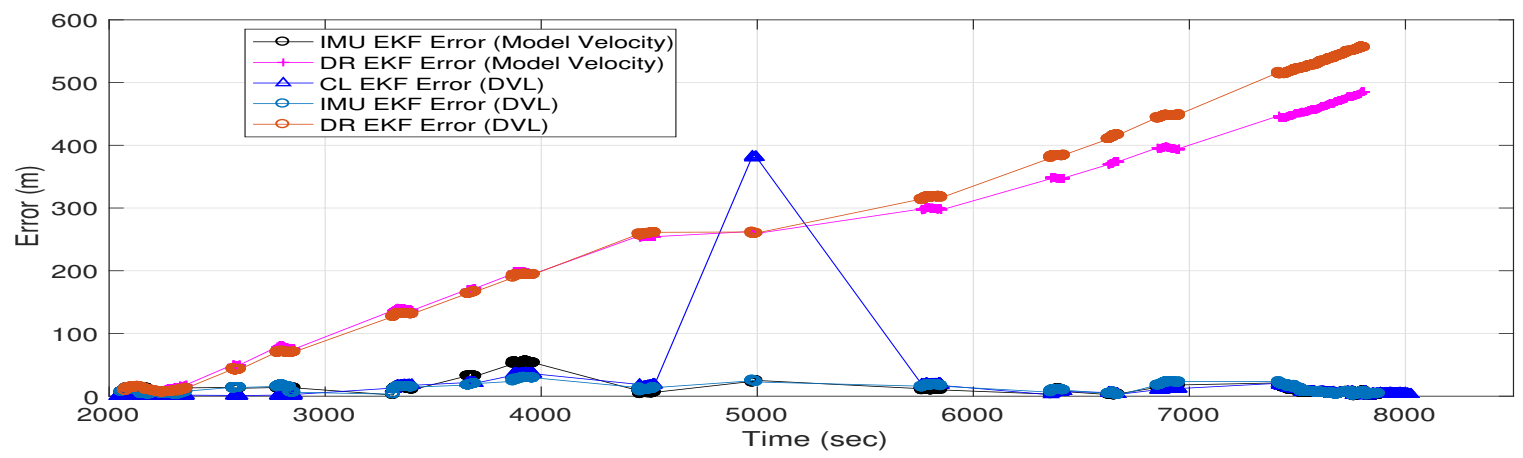

(a) Iver-106 EKF Error Summary - Full View

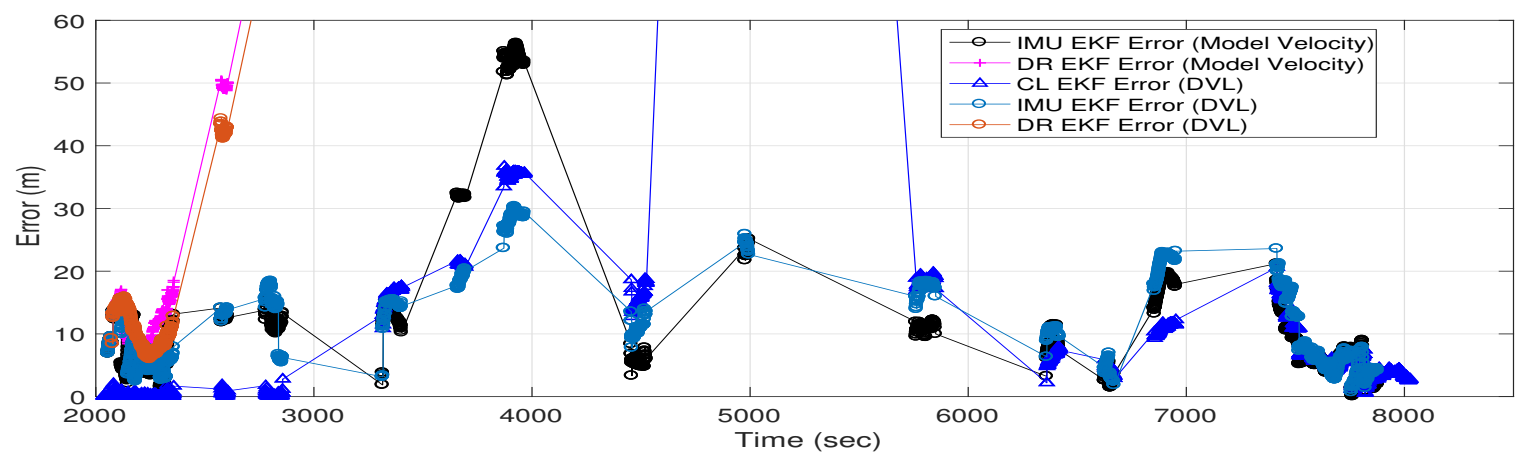

(b) Iver-106 EKF Error Summary - Focused View

Figure 4-17: Iver-106 EKF errors with respect to GPS position at time of the GPS fixes. Displayed in these graphs are the following solutions: model velocity-aided IMU EKF (black), model velocity-aided DR EKF (magenta), DVL-aided CL EKF (dark blue), DVL-aided IMU EKF (light blue), DVL-aided DR EKF (red). Note the difference in scales between the two plots - the full view plot (top) is presented in a focused view (bottom) to highlight the error differences between the solutions. The CL EKF only incorporated GPS until $t=3000$ seconds, hence is the reason why its error is minimal during that time.

Table 4.12: Iver-136 IMU EKF Parameter Values

(a) Model Velocity-Aided

\begin{tabular}{|c|c|}
\hline Parameter & Value \\
\hline$\sigma_{\text {vel }}$ & $1.0 \mathrm{~m} / \mathrm{s}$ \\
\hline$d t$ & $0.042 \mathrm{~s}$ \\
\hline Max Speed & $1.54 \mathrm{~m} / \mathrm{s}$ \\
\hline
\end{tabular}

(b) DVL Aided

\begin{tabular}{|c|c|}
\hline Parameter & Value \\
\hline$\sigma_{\text {vel }}$ & $1.0 \mathrm{~m} / \mathrm{s}$ \\
\hline$d t$ & $0.042 \mathrm{~s}$ \\
\hline Max Speed & $1.54 \mathrm{~m} / \mathrm{s}$ \\
\hline
\end{tabular}


The IMU EKF observed its maximum error of approximately 150 meters in the beginning

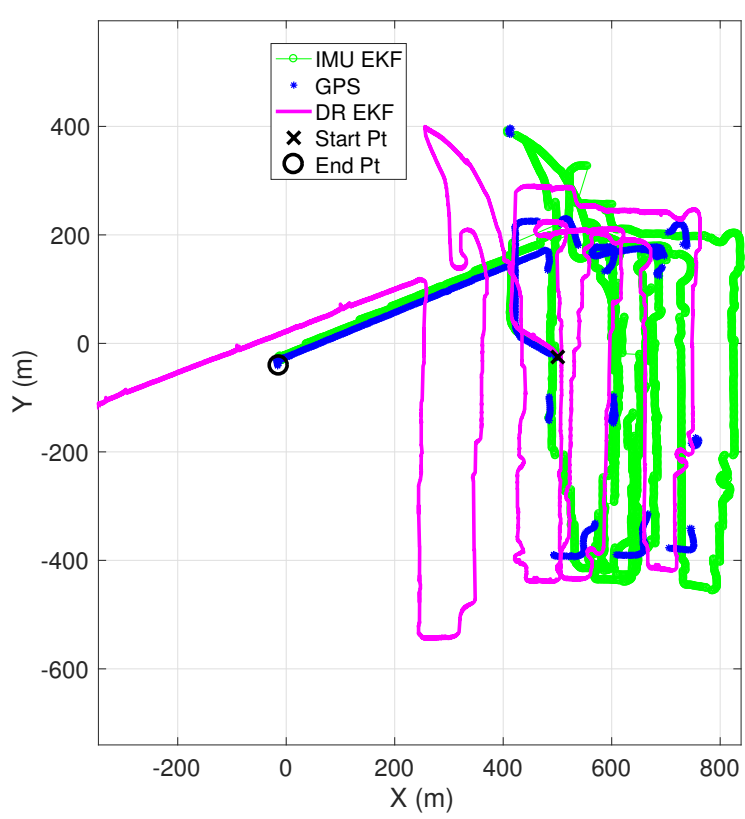

(a) Iver-136 IMU EKF Trajectory - Model Velocity

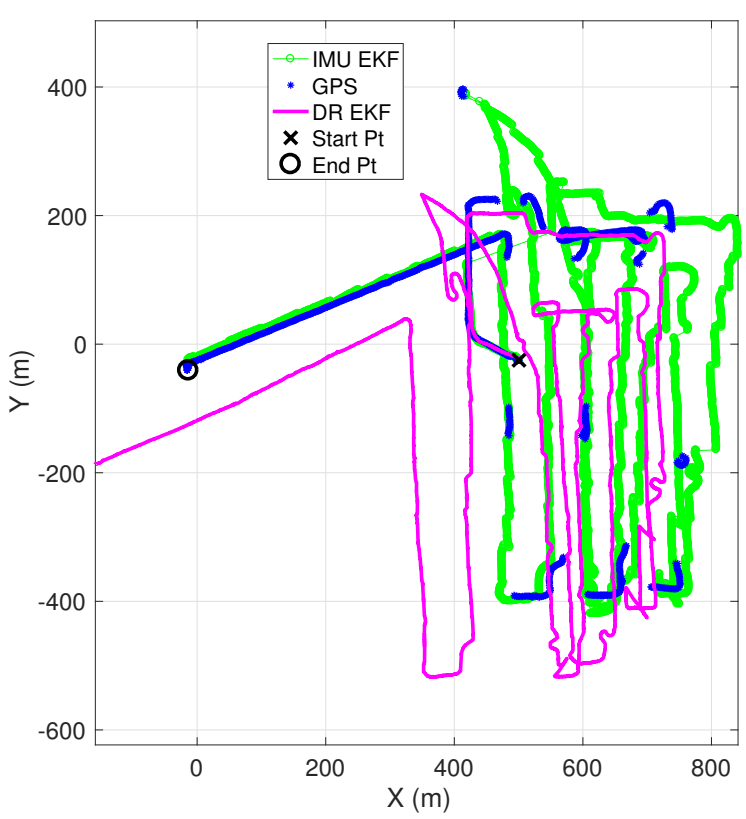

(b) Iver-136 IMU EKF Trajectory - DVL

Figure 4-18: Iver-136 IMU EKF Trajectory Summary. [Left] IMU EKF with the vehicle dynamic model velocity used as its speed measurement. [Right] IMU EKF with the DVL used as the speed measurement.The IMU EKF solution (green) and the DR EKF (magenta) plotted against the associated GPS position fixes (blue) for comparison.

of the mission ( $\mathrm{t}=300$ seconds). This issue occurred both in the model velocity-aided and DVL-aided scenarios. Iver-136 received and processed a range of 716.1 meters at time, $\mathrm{t}=$ 286.7 seconds, from Iver-106 when its state was not accurate, thus resulting in a position shift of Iver-136 to the north between waypoints 2 and 4, as shown in Figure 4-18. The coupled range filter did not prevent this range from being processed because the time and position of the last update used in the range filter algorithm was the initialization point to GPS at position $(500,-25)$ at time, $\mathrm{t}=0$. A speed of $0.88 \mathrm{~m} / \mathrm{s}$ was necessary to trip the state acceptance gate equation in order to prevent the IMU EKF from processing this range. Due to this initial offset, the position estimates remained to the east of the GPS fixes between waypoints 6 to 11, but the IMU EKF later converged to the GPS points at approximately 1100 seconds for both the model velocity-aided DVL-aided IMU EKF. The CL EKF did not experience this same issue because it was in constant receipt of GPS at this time and thus was not affected by the poor state estimates from Iver-106. Nonetheless, the IMU EKF did reconverge and maintained its error below the $2 \sigma$ uncertainty line after $\mathrm{t}=1500$ seconds 


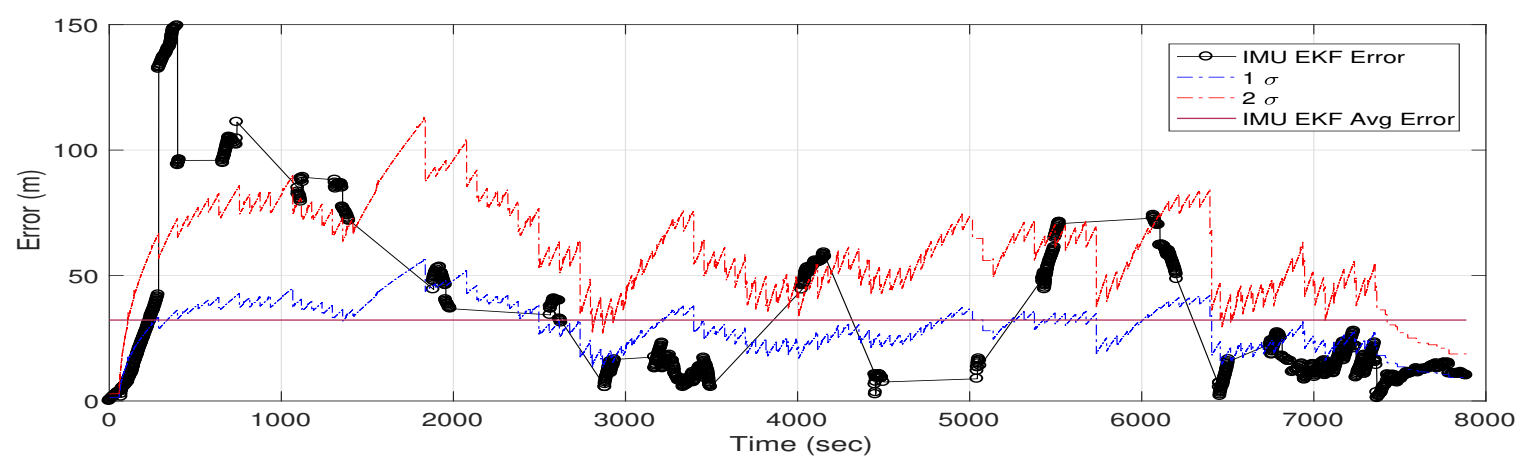

(a) Iver-136 IMU EKF Error - Model Velocity

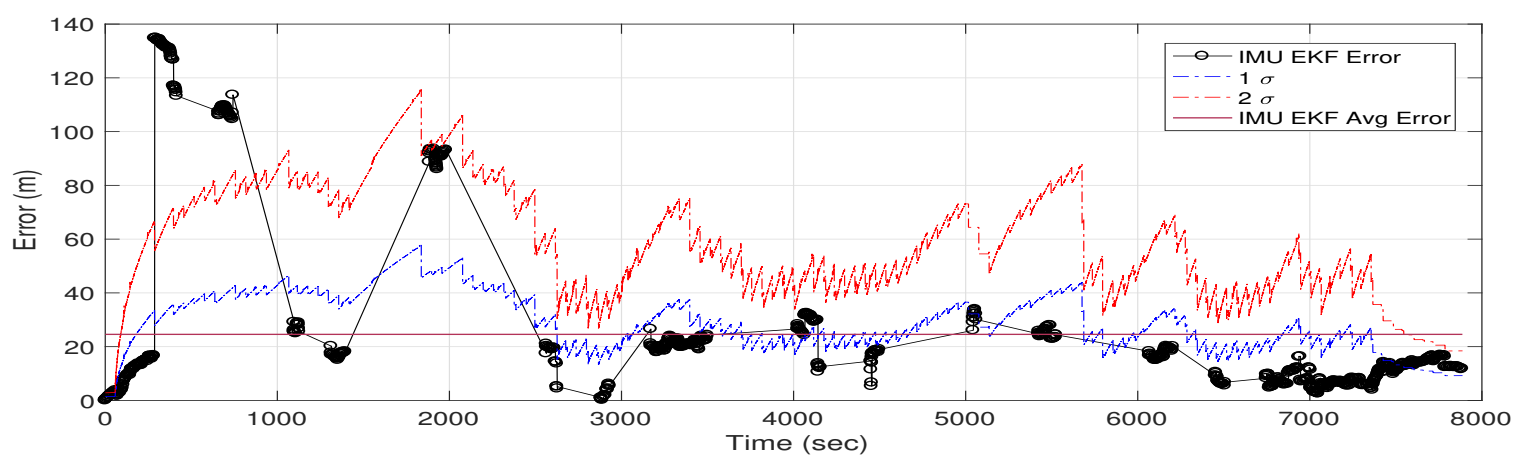

(b) Iver-136 IMU EKF Error - DVL

Figure 4-19: Iver-136 IMU EKF Error Summary. [Top] IMU EKF error with model velocity as the speed measurement. [Bottom] IMU EKF error with DVL used as the speed measurement. The plots contain the IMU EKF error (black), its average error (brown), and its associated $1 \sigma$ (blue) and $2 \sigma$ (red) uncertainty lines.

for the remainder of the mission for both scenarios. Additionally, the IMU EKF did not process the faulty range from the topside beacon at $\mathrm{t}=1000$ seconds, which contributed to the high error of 1600 meters for the CL EKF as shown in Figure 4-20. Likewise, the IMU EKF did not display the erratic behavior on its repeat leg between waypoints 20 and 21 as shown in Figure 4-13b.

Figure 4-20 shows the time series plots of all the EKF errors, and Table 4.13 displays the summary of the average error from GPS for all the EKF solutions. The small average error for the CL-EKF is a poor comparator due to it processing GPS, which essentially reduces the average error close to zero meters. Excluding points from the CL EKF after it processes the GPS fixes provides a more accurate comparison of the IMU EKF error to the CL EKF error. With the exclusion of all error values less than 5 meters, the average CL EKF error was 52.86 meters. The threshold of 5 meters was used based on twice the value of the average GPS position uncertainty of approximately 2.5 meters. 


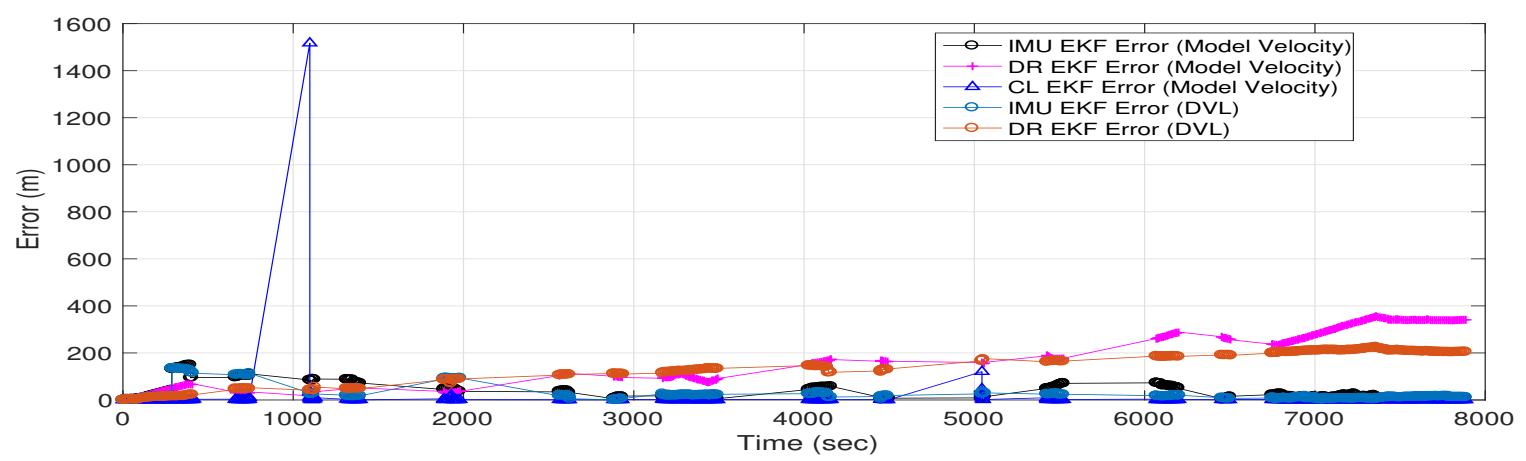

(a) Iver-136 EKF Error Summary - Full View

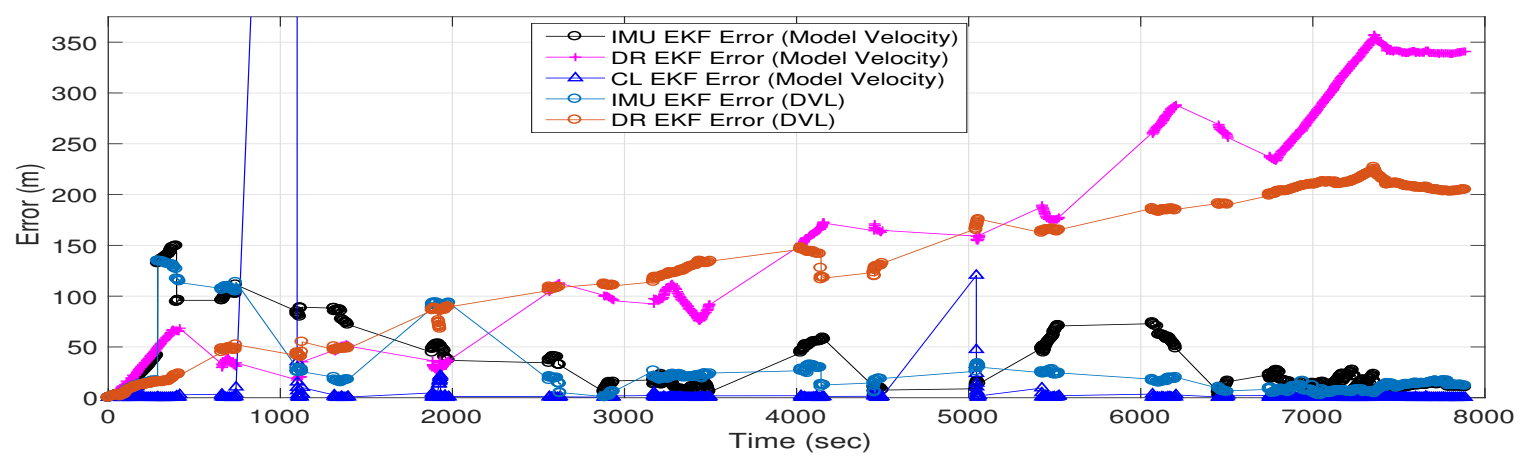

(b) Iver-136 EKF Error Summary - Focused View

Figure 4-20: Iver-136 EKF errors with respect to GPS position at time of the GPS fixes. Displayed in these graphs are the following solutions: model velocity-aided IMU EKF (black), model velocity-aided DR EKF (magenta), model velocity-aided CL EKF (dark blue), DVLaided IMU EKF (light blue), DVL-aided DR EKF (red). Note the difference in scales between the two plots - the full view plot (top) is presented in a focused view (bottom) to highlight the error differences between the solutions. As noted, the model velocity-aided CL EFK (light blue) incorporated GPS, thus its error is minimal as expected.

Table 4.13: Iver-136 EKF Error from GPS Performance Summary

\begin{tabular}{|c|c|c|}
\hline Solution & DVL-Aided & Model Velocity-Aided \\
\hline IMU EKF & $24.57 \mathrm{~m}$ & $32.25 \mathrm{~m}$ \\
\hline CL EKF & $52.86 \mathrm{~m}$ & $\mathrm{~N} / \mathrm{A}$ \\
\hline DR EKF & $142.44 \mathrm{~m}$ & $190.09 \mathrm{~m}$ \\
\hline Distance Traveled & $6.91 \mathrm{~km}$ & $6.91 \mathrm{~km}$ \\
\hline
\end{tabular}

\section{IMU EKF Coupled Range Filter Performance}

This field experiment examined the performance of the IMU EKF coupled range filter, and this section presents a detailed analysis on its performance. For Iver-106, to provide a more accurate comparison of the IMU EKF coupled range filter with the CL EKF, the results from the DVL-aided IMU EKF are used herein. Similarly, with Iver-136, results from the 
model velocity-aided IMU EKF are used since the CL EKF did not use its DVL. The IMU EKF trajectory plots in Figures 4-15b and 4-18a highlight the necessity for the coupled range filter since these plots do not exhibit the same erratic behavior shown in the CL EKF trajectory plots (Figure 4-13). In Figure 4-21, the raw observed OWTT ranges from both beacons for both vehicles are displayed with those ranges processed by the coupled range filter. The coupled range filter removed the extraneous ranges that are due to inadequate clock synchronization or variable acoustic environment at $t=4700$ seconds for Iver-106 and $\mathrm{t}=280$ seconds and $\mathrm{t}=1000$ seconds for Iver-136. Also, this plot shows the effectiveness of the state acceptance gate since it removed the extraneous ranges due to poor beacon state estimates. Iver-106 exhibited the most erratic behavior with its CL EKF while transiting between waypoints 19 to 22. As shown in Figure 4-21a, the coupled range filter prevented Iver-106 from processing those ranges from Iver-136 from time, $\mathrm{t}=4500$ seconds, to time, $\mathrm{t}=5500$ seconds. In similar fashion, for Iver-136, the most erratic behavior was observed from $\mathrm{t}=4500-5000$ seconds while transiting from waypoints 20 to 21 . These ranges from Iver-106 were not processed from 4500 to 6000 seconds, as shown in Figure 4-21b.

Time series plots of the individual $\mathrm{X}$ and $\mathrm{Y}$ position estimates for both the IMU EKF and CL EKF, compared to the GPS fixes provide a good assessment on the coupled range filter's performance. These plots, shown in Figure 4-22 for both Iver-106 and Iver-136, indeed show the effectiveness of the coupled range filter. For Iver-106, the erroneous behavior from the CL EKF from 4700 to 5200 seconds was replaced by a more constant trajectory exhibited by the IMU EKF during the same time period. The IMU EKF for Iver-136 showed improved performance from the CL EKF as well. At 1000 seconds, the IMU EKF did not process the faulty range from the topside beacon, thus its position estimate was not immediately offset more than 1000 meters to the northeast. In addition, from 4700 to 5100 seconds, the IMU EKF produced an improved trajectory instead of the irregular behavior demonstrated by the CL EKF.

Statistics on the coupled range filter are summarized in Table 4.14. The vehicles observed an acoustic packet loss of $14.22 \%$ and $23.16 \%$ for Iver-106 and Iver-136, respectively. Of these observed ranges, the IMU EKF coupled range filter removed $6.63 \%$ of the received ranges for Iver-106 and $11.88 \%$ received ranges for Iver-136. 

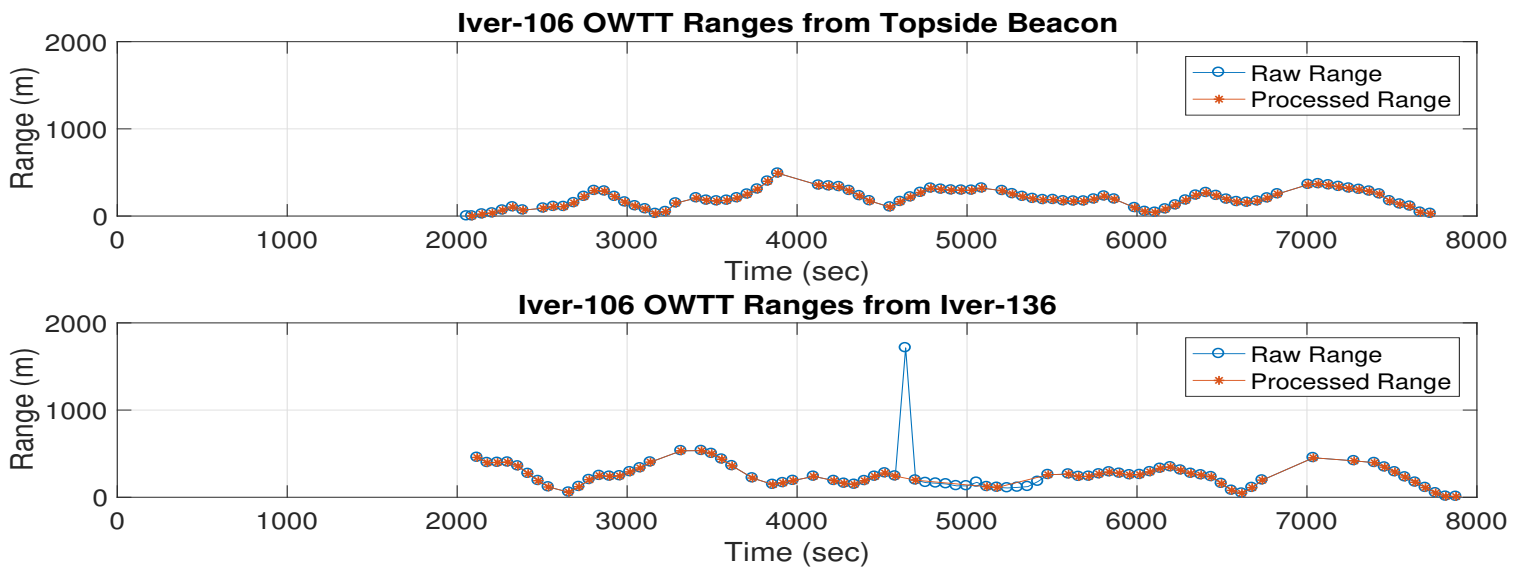

(a) Iver106 Range Measurement Summary
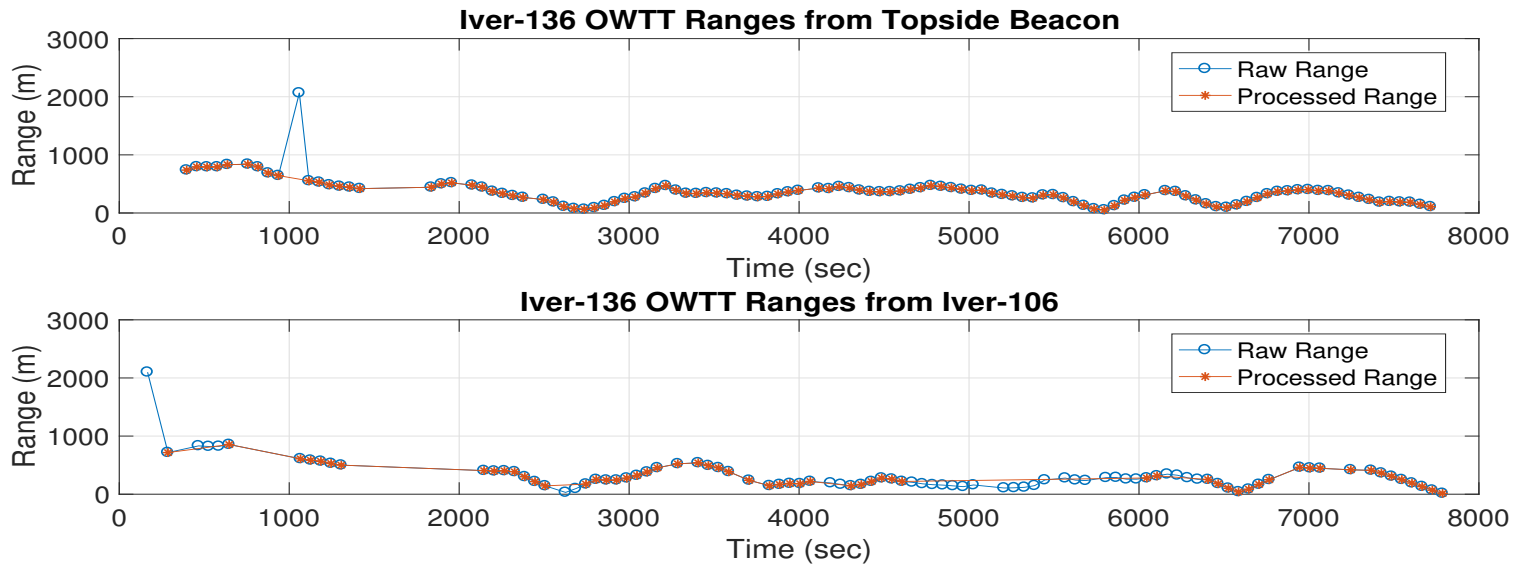

(b) Iver136 Range Measurements Summary

Figure 4-21: IMU EKF Range Measurement Time Series Summaries. Iver-106 (top) and Iver-136 (bottom) raw and IMU EKF processed OWTT range measurements. These plots show the raw observed OWTT range measurements (red) and the processed OWTT range measurements from the coupled range filter (blue) from both the topside beacon and from the other vehicle.

Table 4.14: IMU EKF Coupled Range Filter Performance Summary

\begin{tabular}{|c|c|c|}
\hline Parameter & Iver-106 (DVL) & Iver-136 (M-V) \\
\hline Acoustic Packet Loss & $14.44 \%$ & $23.16 \%$ \\
\hline No. of Raw OWTT Ranges & 166 & 202 \\
\hline No. Removed by Pre-Range Filter & 1 & 2 \\
\hline No. Removed by Range Speed-Rate Filter & 11 & 30 \\
\hline Total Percent of Ranges Removed & $7.23 \%$ & $15.84 \%$ \\
\hline
\end{tabular}

\subsection{Discussion}

These three controlled in-water field trials presented in the preceding sections provide an extensive analysis of the IMU EKF with the coupled range filter by using three different 

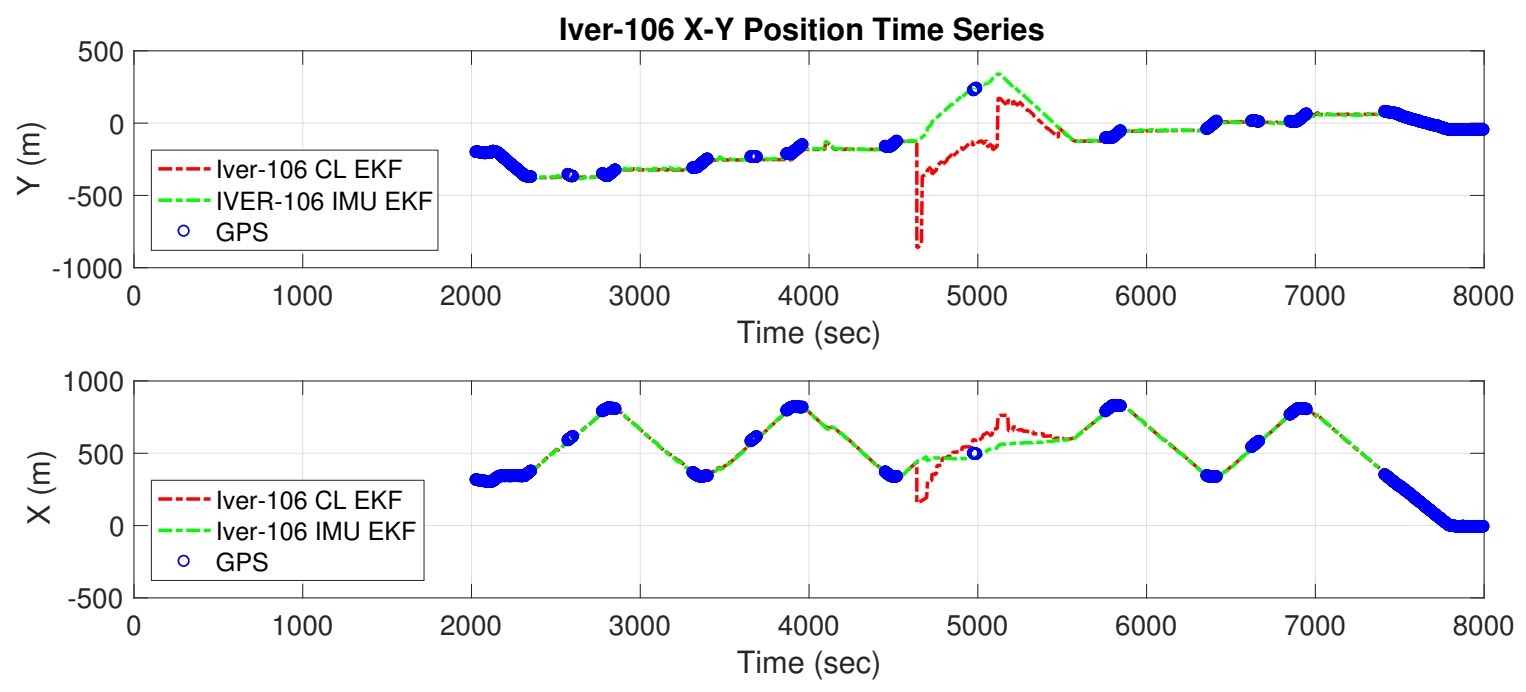

(a) Iver-106 XY Position Time Series Plot
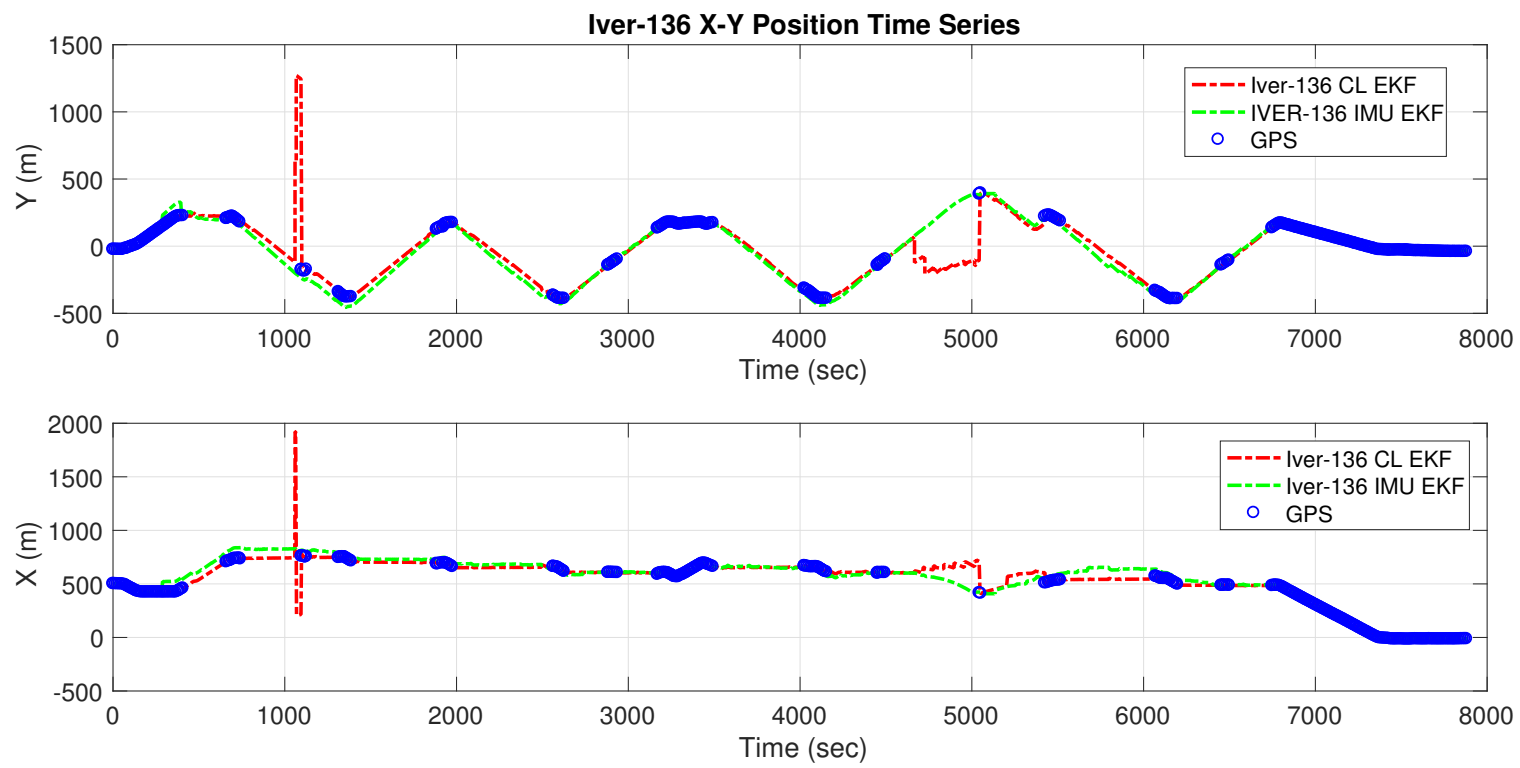

(b) Iver-136 XY Position Time Series Plot

Figure 4-22: Iver-106 \& Iver-136 Coupled Range Filter Performance. Iver-106 (top) and Iver-136 (bottom) X and Y time series plots of IMU EKF (green) plotted with the CL EKF (red) and associated GPS position fixes (blue). These plots show the necessity of the coupled range filter to remove the erratic behavior displayed by the CL EKF. The difference in scales between the plots signifies the degree of erratic position estimation without the coupled range filter.

vehicles executing three different mission trajectories in two different locations. Compared to other navigation methods presented in this research, the IMU EKF is a competitive alternative to the current approaches. Since the main innovation to this EKF is the use of linear accelerations as an odometry input with OWTT range measurements, some unique 
issues were discovered to prevent instability and singularity of the IMU EKF. The following discussion in this section pertains to the experiments discussed in Sections 4.1 - 4.3.

\subsubsection{IMU Linear Acceleration Analysis}

Since MEMS IMUs inherently exhibit high noise in their acceleration measurements, the IMU EKF must utilize some form of a velocity measurement to remain stable. The vehicle's IMU senses non-zero accelerations (because of noise) even when the vehicle is stopped or moving at a constant velocity. These non-zero accelerations, which do not reflect the actual dynamics of the vehicle, are then integrated and double integrated to determine velocity and position, respectively. Therefore, without a velocity measurement, the IMU EKF estimated position becomes unstable. A solution to the problem was to restrict linear acceleration measurements to the IMU EKF only while velocity measurements (either model velocity or DVL) were available. Velocity measurements of zero or non-zero value, with the linear acceleration measurements, were sufficient for the IMU EKF to maintain stability. For the data presented in this work, linear acceleration measurements were prevented from being infused to the IMU EKF until the vehicle received a velocity measurement at the start of the mission. Likewise, the IMU EKF did not process any acceleration measurements at the end of the mission when velocity measurements were no longer available.

\subsubsection{Singularity Analysis}

During the measurement update step of the Kalman Filter process, an inversion of a square matrix, $\left(\mathbf{R}+\mathbf{H P}^{-} \mathbf{H}^{\mathrm{T}}\right)$, is conducted to determine the Kalman Gain, $\mathbf{K}$, as shown in Equation (2.9). A valuable measure of effectiveness is a numerical analysis of how close the IMU EKF approaches singularity, for this quantity indicates the level of robustness of the IMU EKF. The determinant of the square matrix, $\left(\mathbf{R}+\mathbf{H P}^{-} \mathbf{H}^{\mathrm{T}}\right)$, was calculated for all of the model velocity-aided IMU EKF solutions in all three field experiments. Assessing the values of this determinant provides insight on how close the IMU EKF comes to a singularity.

In all three field experiments presented in this work totaling 407,895 computations by the IMU EKF, one instance of a singularity was observed, which occurred when the first received measurement is a OWTT measurement. With certain values for initialization for the estimated covariance matrix, $\mathbf{P}_{k}^{+}$, the standard KF equations for the predicted and measurement steps will eventually lead to off-diagonal values in the $\mathbf{P}_{k}^{+}$matrix approaching 
negative infinity. Thus, when the IMU EKF attempts the matrix inversion in Equation 2.9, a singularity is imminent. This issue was observed only in the Ashumet Pond trials of October 2016 when the coupled range filter was not implemented. Thus, when implementing the IMU EKF on that particular field data from October 2016 for Iver-106 only, the first two range measurements (one from the topside beacon and one from Iver-136) were removed to prevent the singularity. Both the Charles River and Ashumet Pond April 2017 field trials utilized the coupled range filter, which removed these ranges from being processed and thus prevented any singularity from occurring. Thus, no range measurements were removed from those field data sets even though OWTT ranges existed as the first measurements available to the IMU EKF.

With the exception of this one observation, the IMU EKF maintained a large margin from a singularity as observed in all three field experiments. Figure 4-23 provides one example of a time series summary of the determinant value of the $\left(\mathbf{R}+\mathbf{H P}^{-} \mathbf{H}^{\mathrm{T}}\right)$ over the course of the entire field trial. For the remainder of the other model velocity-aided IMU EKF solutions, Table 4.15 provides a summary of statistics on the determinant value. Based on these observations and results, this numerical analysis provides a necessary, but not sufficient, conclusion that the IMU EKF maintains a considerable margin to a singularity. As already discussed, the dead-reckoned solution (i.e., DR EKF) propagates the position error at a mostly linear rate, which is better than having the IMU EKF processing a faulty range and thus becoming unstable or singular.

Table 4.15: IMU EKF Numerical Singularity Analysis Summary

\begin{tabular}{|c|c|c|c|c|c|}
\hline $\begin{array}{c}\operatorname{det}\left(\mathbf{R}+\mathbf{H P}^{-} \mathbf{H}^{\mathrm{T}}\right) \\
\text { Parameter }\end{array}$ & $\begin{array}{c}\text { Iver-106 } \\
\text { Oct'16 }\end{array}$ & $\begin{array}{c}\text { Iver-136 } \\
\text { Oct'16 }\end{array}$ & $\begin{array}{c}\text { SandShark } \\
\text { Oct'16 }\end{array}$ & $\begin{array}{c}\text { Iver-106 } \\
\text { Apr'17 }\end{array}$ & $\begin{array}{c}\text { Iver-136 } \\
\text { Apr'17 }\end{array}$ \\
\hline Minimum & $1.38 \mathrm{e}-3$ & $1.53 \mathrm{e}-3$ & $1.23 \mathrm{e}-4$ & $7.35 \mathrm{e}-3$ & $1.78 \mathrm{e}-3$ \\
\hline Average & 2.17 & 2.41 & 0.67 & $2.25 \mathrm{e}+3$ & $1.47 \mathrm{e} 3$ \\
\hline Maximum & $3.79 \mathrm{e}+3$ & $5.50 \mathrm{e}+3$ & 21.00 & $2.49 \mathrm{e}+4$ & 1.77 \\
\hline Number of Samples & 38,637 & 47,999 & 65,610 & 68,597 & 187,052 \\
\hline $1 \sigma$ & 46.36 & 55.51 & 1.89 & $5.01 \mathrm{e}+3$ & 14.33 \\
\hline
\end{tabular}

\subsubsection{Performance Summary}

A summary of the performance metrics discussed in this thesis for all of the navigation solutions are shown in Figures 4-24a for 2016 field data and Figure 4-24b for the 2017 field 


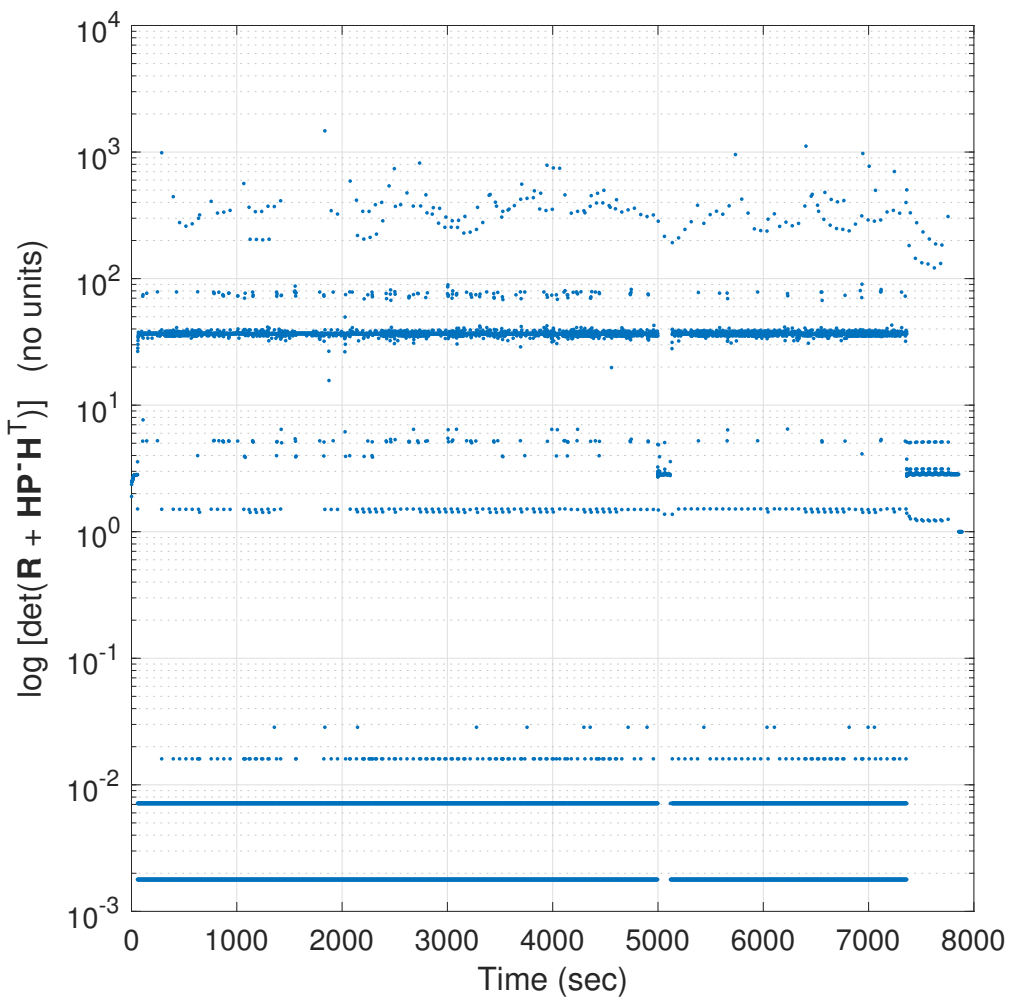

Figure 4-23: April 2017 Iver-136 Singularity Analysis. This plot displays the value of the determinant of $\left(\mathbf{R}+\mathbf{H P}^{-} \mathbf{H}^{\mathrm{T}}\right)$, the inverted portion of the Kalman Gain Matrix, $\mathbf{K}$, for the model velocity-aided IMU EKF on Iver-136. Throughout the entire field trial, the IMU EKF does not approach singularity.

data. These bar graphs present all of the different navigation solution average errors from the respective GPS locations with their associated $1 \sigma$ standard deviations. In these bar charts, for the closed-loop solutions that utilized GPS (SandShark in 2016 (Figure 4-24a) and Iver136 in 2017 (Figure 4-24b)), the error values after the CL solution had adjusted to GPS have been removed to provide a more accurate comparison to other solutions. As these charts show, the IMU EKF displayed superior performance in both the 2016 and 2017 field trials with minimum average error and standard deviations. Even when the IMU EKF displayed equal performance with the CL EKF in the metric of average position error, as shown with the Iver-106 solutions in 2017, the $1 \sigma$ error values for the IMU EKF were drastically smaller than those of the CL EKF. The model velocity-aided IMU-EKF $1 \sigma$ error was $33.81 \%$ lower, and the DVL-aided IMU EKF was 82.09\% lower than the Iver-106 DVL-aided CL EKF $1 \sigma$ value for the April 2017 field experiment. 


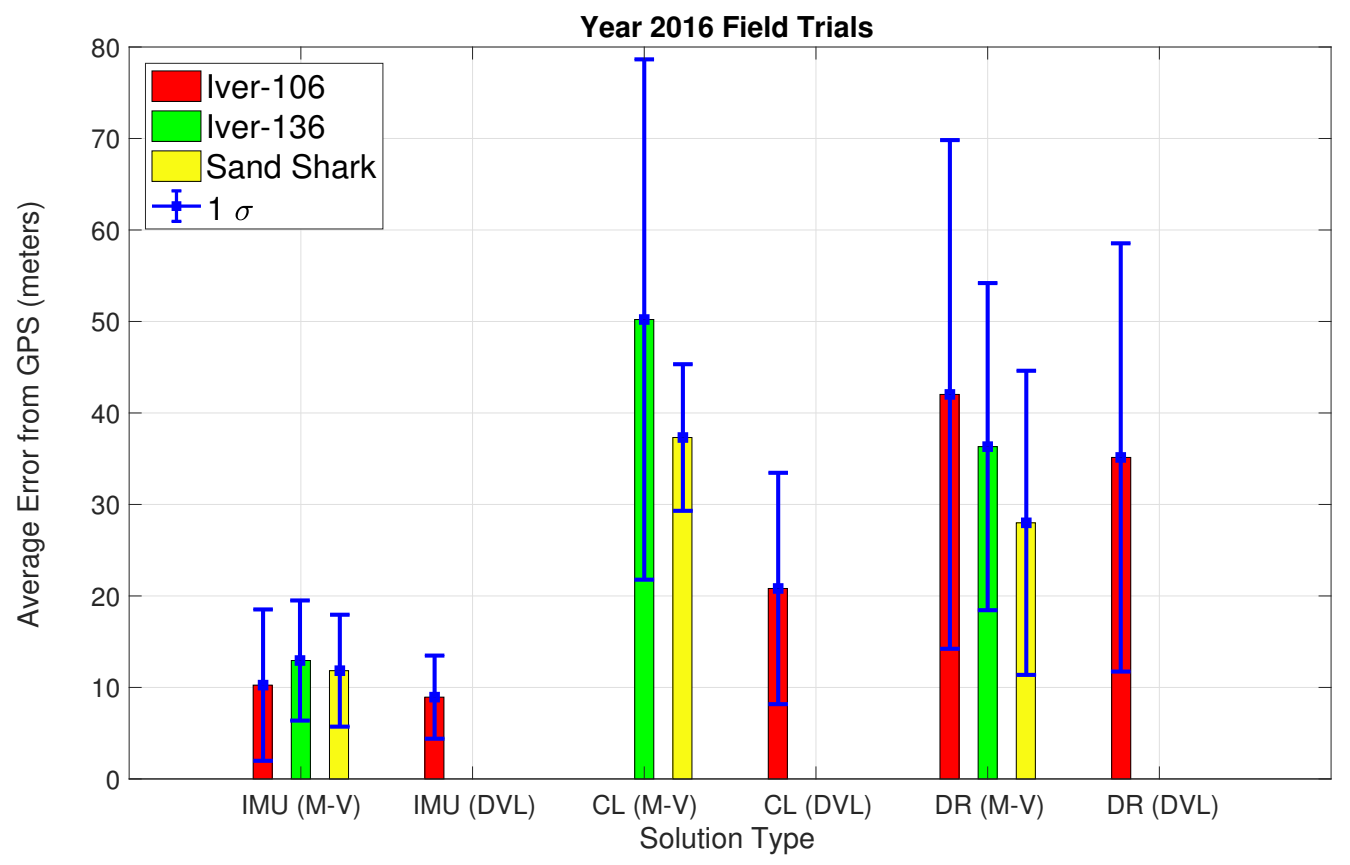

(a) Year 2016 Navigation Solution Performance Summary

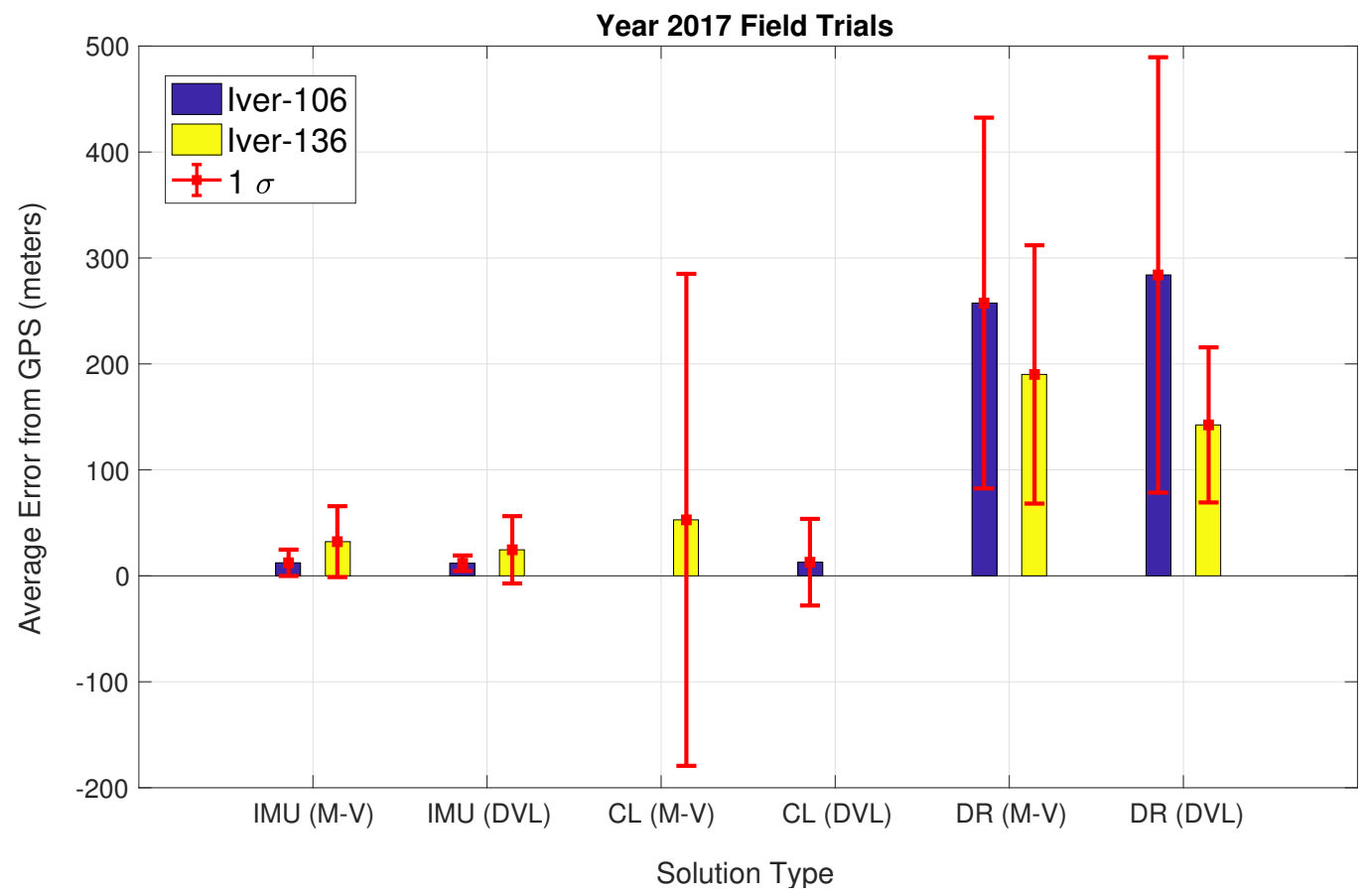

(b) Year 2017 Navigation Solution Performance Summary

Figure 4-24: [Top] Year 2016 Field Trials Performance Summary. Each EKF solution (IMU, CL, and DR) is displayed for Iver-106 (red), Iver-136 (green), and SandShark (yellow) with associated standard deviations (blue). [Bottom] Year 2017 Field Trials Performance Summary. Each EKF solution (IMU, CL, and DR) is displayed for Iver-106 (blue) and Iver-136 (yellow) with associated standard deviations (red). "M-V," and, "DVL," in parenthesis represent the respective model velocity and DVL-aided solutions. 


\subsection{Monterey Bay Trials - September 2016}

Field data from the Keck Institute for Space Studies "Satellites to the Ocean Floor," research field program [44] served as a final implementation of the IMU EKF. As part of this field program, Iver-136 conducted a mission in Monterey Bay, CA (see Figure 4-25), and data gathered during this mission provides the analysis for this thesis. Since one of the focuses of this thesis is navigation without a DVL, this field data provides a good assessment of the model velocity-aided IMU EKF implemented on a vehicle operating in strong currents and in water depths where DVL bottom-lock is not available.

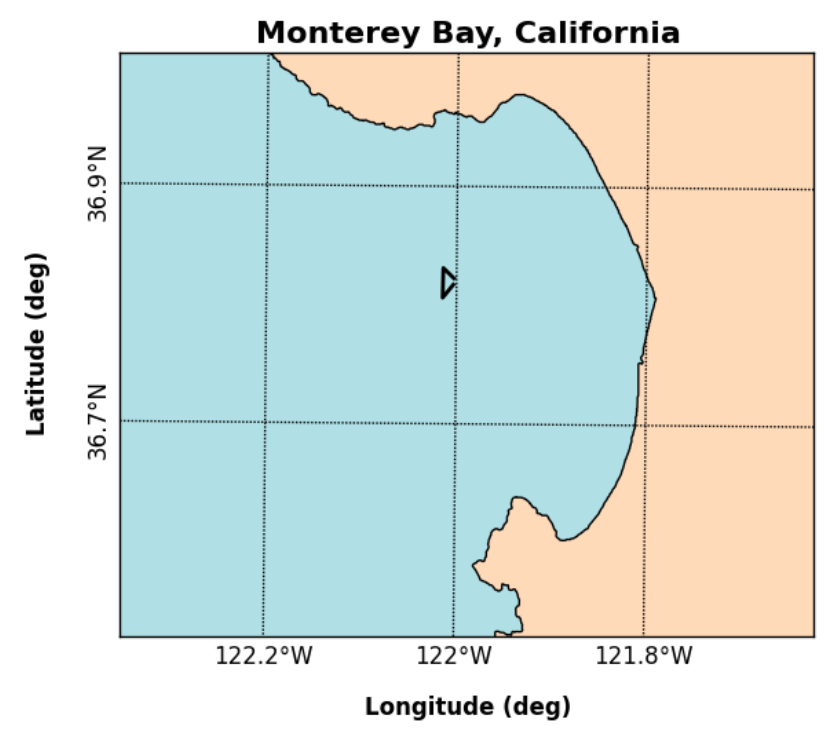

Figure 4-25: Monterey Bay, California with position of Iver-136 mission, identified by the black triangle, during the Keck Institute for Space Studies field program.

Since ocean currents are prevalent in Monterey Bay, the addition of a velocity bias estimator similar to one used in a previous work [9] was added to this IMU EKF implementation to aid in making the model velocity input more accurate. In the previous work as well as other work involving underwater gliders operating in the Arctic Ocean [55], the state updates from the OWTT range measurements provided a means of calculating a water velocity measurement. This water velocity measurement is then processed in a coupled Kalman Filter to the navigation state estimator that produces an updated velocity bias. The model velocity measurement incorporates this bias, thus updating it with ocean currents and making it 
more accurate.

\subsubsection{Trajectory Summary}

During this field survey, Iver-136 operated in a half-bow-tie type trajectory, as shown in Figure 4-26. This plot shows three solutions for comparison (IMU EKF, DR EKF, and the CL EKF) along with GPS points during the surfacings in the mission. Data was trimmed to the time line of the vehicle's deployment. Since this mission involved a single beacon (and similar to the analysis in the "Bias Estimation \& Inter-vehicle Range Comparison," analysis of Section 4.1.2), all of the parameters identified in Table 4.2 remained the same except the process noise gain factor, $\tilde{q}$, was set to 100 versus 1.0. Unlike the previous experiments, the CL EKF solution did not incorporate any OWTT range measurements, but it did process GPS and used the model velocity for its speed estimation. However, similar to the implementation in Section 4.1.2, the IMU EKF in this implementation used a more accurate model velocity than the one used for the CL EKF.

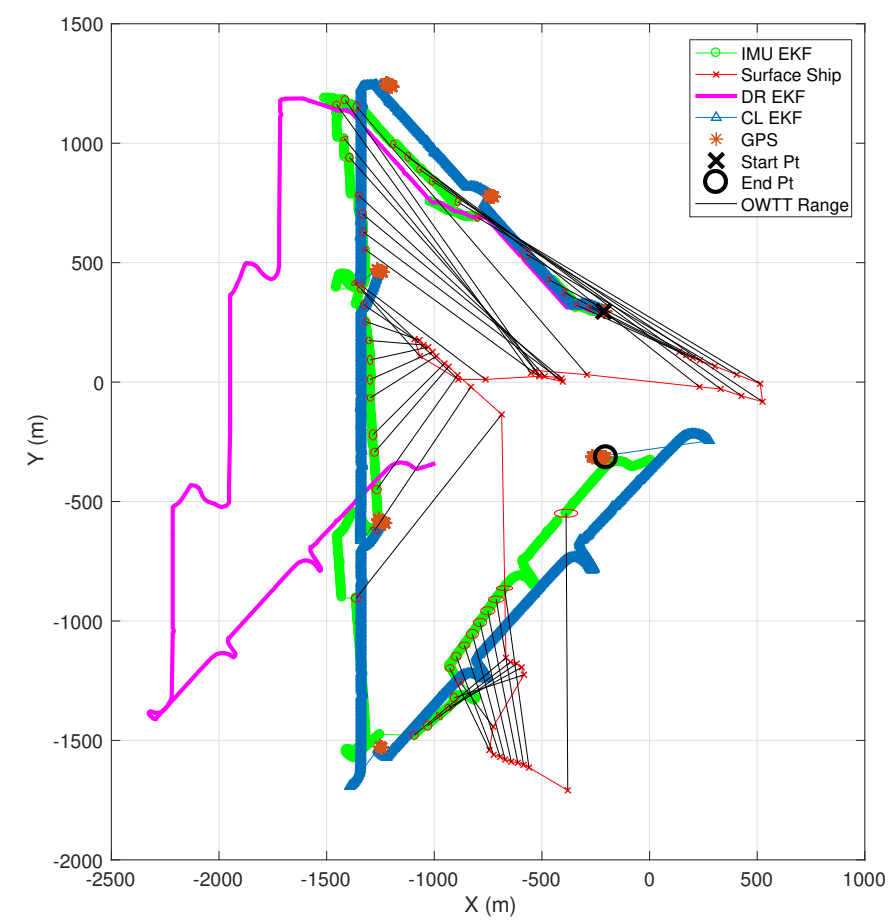

Figure 4-26: Iver-136 trajectory summary on Monterey Bay, CA. The following navigation solutions are displayed: IMU EKF (green), CL EKF (light blue), and the DR EKF (magenta). GPS (brown) fixes are displayed for ground truth. The R/V Shana Rae, which served as the surface beacon (red), maneuvered throughout the mission to maintain acoustic connectivity. The black lines illustrate the OWTT ranges from the surface beacon to the vehicle, and the red ellipses are the position uncertainties computed by the IMU EKF. 


\subsubsection{Performance Analysis}

The total mission time was approximately 103 minutes with a total of 6.1 kilometers traveled. Like the Ashumet Pond trails, the surface beacon was set to transmit at a frequency of once every 60 seconds $(\sim 0.02 \mathrm{~Hz})$. However, Iver-136 only received a total of 49 acoustic packets, equating to an acoustic loss of approximately $52 \%$. Reasons for the high loss rate were due to the $\mathrm{R} / \mathrm{V}$ Shana Rae repositioning during the mission as well as high background noise from its engines, thus lowering the signal to noise ratio of the transmitted packets. Nonetheless, since Iver-136 experienced such a high loss rate of its OWTT measurements, the vehicle accrued significantly more error compared to the previous results discussed in the previous sections.

Figure 4-27 shows the error with respect to the GPS position fixes for various navigation solutions. As shown, because of the high acoustic loss rate, the IMU EKF experienced an average error of approximately 141 meters. However, this plot does show the significant effect of the velocity bias estimator. The IMU EKF that incorporated the velocity bias estimator (light blue) outperforms the IMU EKF solution without it (red) by approximately $30 \%$, particularly from $\mathrm{t}=4000$ seconds to the end of the mission. Additionally, when the IMU EKF processed GPS (gold), it exhibited a better position reset offset as compared to the CL EKF (purple). Particularly, at $\mathrm{t}=5667$ seconds, the IMU EKF had a position reset of approximately 200 meters less than the CL EKF. Likewise, at $\mathrm{t}=7770$ seconds, the IMU EKF experienced a position reset of approximately 250 meters less than the CL EKF. Hence, overall, the IMU EKF method presents a better alternative to the CL EKF solution.

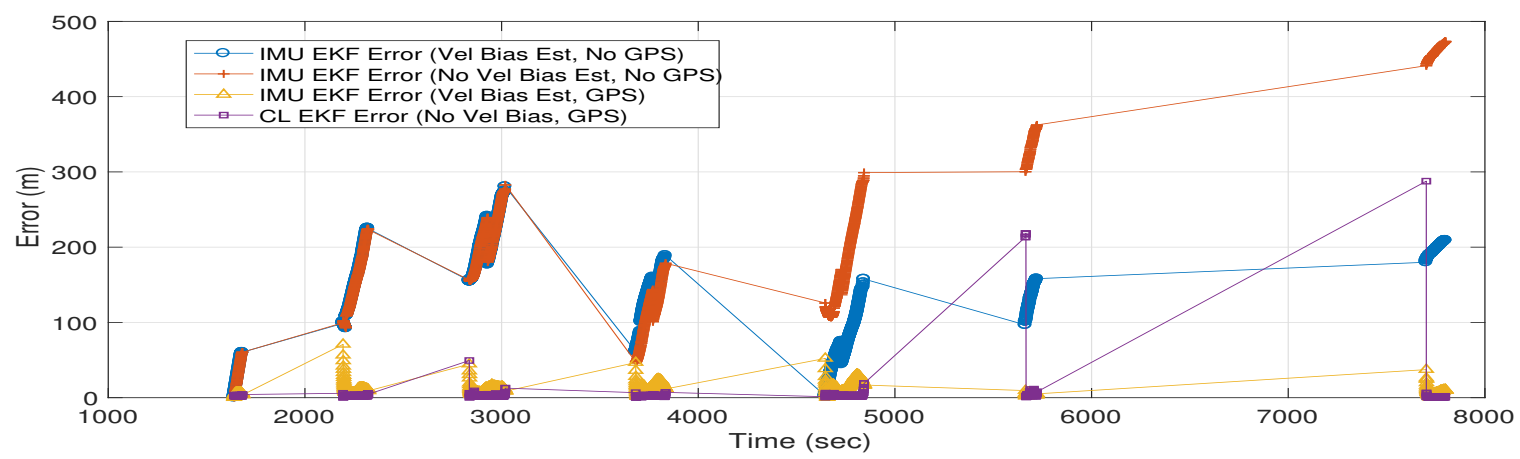

Figure 4-27: Iver-136 Navigation Solution Error summary. Navigation solution error compared to the GPS fixes are displayed: IMU EKF with velocity bias estimator (blue), IMU EKF without the velocity bias estimator (red), IMU EKF with velocity bias estimator and GPS (gold), and the CL EKF (purple). 
THIS PAGE INTENTIONALLY LEFT BLANK 


\section{Chapter 5}

\section{Conclusion}

\subsection{Research Summary}

The main goal of this thesis was to investigate a navigation solution for autonomous underwater vehicles that used a minimal sensor suite to reduce power consumption and cost, allow applicability throughout the water column, and perform with an acceptable threshold of accuracy. The research presented in this paper proves a viable option exists that is dependent upon a MEMS IMU for odometry and attitude, a vehicle's dynamic model velocity, and passive acoustic aiding.

In this navigation solution, the vehicle's state was estimated by an EKF that propagated a kinematic constant acceleration model to determine the vehicle's XY position, velocity, and acceleration. This propagated model was augmented by processing measurements of linear accelerations from the MEMS IMU, velocity from propeller motor speed or a DVL, and OWTT range measurements from one or two beacons. Also, the estimated state included IMU accelerometer bias, which incorporated bias measurements determined from the state's updated position after a range measurement. Coupled with the EKF was a range filter that prevented the EKF from processing erroneous OWTT range measurements either because of inaccurate clock synchronization between the beacon and the receiver, inaccurate state estimation from the transmitting beacon, or due to various environmental conditions that can affect sound propagation. This range filter prevented the IMU EKF from processing faulty ranges by two processes: (1) a range threshold filter, and, (2) a state acceptance gate.

The effectiveness of this EKF model was evaluated by real-time implementations on data collected in three different controlled environment field experiments. These field experiments 
included two OceanServer Iver2 AUVs in two separate field trials on Ashumet Pond and one field experiment of a Bluefin Robotics SandShark $\mu$ AUV on the Charles River. Each of these experiments incorporated a topside beacon, transmitting its GPS position, while the two experiments in Ashumet Pond also incorporated an assessment of inter-vehicle ranging and multi-vehicle navigation. These experiments provided a wide spectrum of different vehicles, different acoustic environments, and different mission trajectories that provided a valuable means of assessing the capability of the IMU EKF navigation method. Analysis of these experiments proves that the IMU EKF method gives an average position error on the order of tens of meters over a time scale of about two hours and a spatial scale of approximately seven kilometers. Additionally, the use of other vehicles in formation as a transmitting beacon and incorporating bias estimation enhanced the navigation performance. Also, the addition of the coupled range filter was paramount to the IMU EKF's success by preventing faulty range measurements that cause the IMU EKF to become unstable or singular. Lastly, an implementation of the IMU EKF in Monterey Bay, CA proved the this method outperforms other current methods in open ocean currents.

\subsection{Recommendations for Future Research}

This research proves that low cost, low power, accurate navigation is attainable. The following are recommendations for improvement and innovation in progressing this area forward:

1. Distinction between velocity bias and accelerometer bias. Due to the first three experiments being performed in essentially zero-current waters, all bias was concluded to be a result from accelerometer bias and not from any velocity bias (i.e., current). For the last experiment in open ocean, the range updates were used to determine both acceleration and velocity bias. Thus, further research is needed on how to distinguish velocity and acceleration bias when using state updates from OWTT range measurements.

2. Long-term MEMS IMU bias assessment. Further research on behavior and modeling of MEMS IMU accelerometer bias and drift will aid in minimizing errors from these sources for longer duration missions (as applied to autonomous underwater gliders, for example). Additionally, investigating other means of modeling IMU accelerometer bias to determine optimal models will further aid in making this solution more accurate. Previous efforts on stochastic modeling of IMU errors indicate the variety of models that can be used [15]. 
3. Update a vehicle's model velocity in real time. For vehicles that contain a DVL, future research should determine how DVL measurements can update the vehicle's propeller motor speed to model velocity mapping table in real time. This research will also aid in determining optimal power consumption (and thus enhancing vehicle endurance) since the DVL is only used when necessary to provide a more accurate model velocity measurement.

4. Experimental determination of a vehicle's model-velocity standard deviation. A more accurate determination of the $1 \sigma$ value for a vehicle's model-velocity should enhance the navigation solution's accuracy.

5. Decision points for vehicle determining poor geometry. With the coupled range filter's state acceptance gate, the vehicle's navigation solution now has a decision point of determining poor geometry. Future research should focus on how best for the vehicle to respond at these decision points. Possible options are notification of human operators by the vehicle, changing the vehicle's course and/or speed for a more optimal geometry, or obtaining a GPS fix to ensure the navigation solution minimizes potential instability.

\subsection{Future Applications}

This navigation solution can be utilized in a variety of applications for underwater vehicles. It is beneficial to the emerging $\mu \mathrm{AUV}$ class and the longer endurance platforms, such as autonomous underwater gliders. Additionally, this solution is attractive for future "masterslave" heterogenous vehicle deployments, in which a large AUV with a high-end inertial navigation system serves as the communications and navigation aid to smaller vehicles [21]. Lastly, this method serves as an additional solution for those vehicles that utilize a DVL but are in areas where DVL bottom-lock is not available, such as diving, ascending, or conducting mid-water column operations.

The future for autonomous underwater vehicle navigation is promising. With proof that inexpensive, low power consumption, yet accurate, navigation methods are available, the ability to deploy multiple vehicles for scientific research and military missions will enable these communities to put their conceptual ideas into action. 
THIS PAGE INTENTIONALLY LEFT BLANK 


\section{Bibliography}

[1] Ashumet Pond Barnstable County Cape Cod Watershed. http://www.mass.gov/eea/ $\mathrm{docs} / \mathrm{dfg} / \mathrm{dfw} / \mathrm{habitat} / \mathrm{maps}-$ ponds/dfwashu.pdf. Accessed: 2017-03-07.

[2] Roberto Alonso and Malcolm D Shuster. TWOSTEP: A fast robust algorithm for attitude-independent magnetometer-bias determination. Journal of the Astronautical Sciences, 50(4):433-452, 2002.

[3] Alexander Bahr, Matthew R Walter, and John J Leonard. Consistent cooperative localization. In IEEE International Conference on Robotics and Automation, 2009. ICRA'09., pages 3415-3422. IEEE, 2009.

[4] Yaakov Bar-Shalom, X Rong Li, and Thiagalingam Kirubarajan. Estimation with Applications to Tracking and Navigation: Theory Algorithms and Software. John Wiley \& Sons, 2001.

[5] Alessandro Benini, Adriano Mancini, Alessio Marinelli, and Sauro Longhi. A biased extended Kalman filter for indoor localization of a mobile agent using low-cost IMU and UWB wireless sensor network. IFAC Proceedings Volumes, 45(22):735-740, 2012.

[6] Robert Grover Brown and Patrick Y.C. Hwang. Introduction to Random Signals and Applied Kalman Filtering with MATLAB Exercises and Solutions. John Wiley \& Sons, 3rd edition, 1997.

[7] Richard S Bucy and Peter D Joseph. Filtering for Stochastic Processes with Applications to Guidance. Chelsea Publishers, 2 edition, 1987.

[8] Brian Claus and Ralf Bachmayer. A parameterized geometric magnetic field calibration method for vehicles with moving masses with applications to underwater gliders. Journal of Field Robotics, 34(1):209-223, 2017.

[9] Brian Claus, James H Kepper IV, Stefano Suman, and James C Kinsey. Closed loop one-way-travel-time navigation using low-grade odometry for autonomous underwater vehicles. Journal of Field Robotics, 2017, Accepted.

[10] John L Crassidis, Kok-Lam Lai, and Richard R Harman. Real-time attitudeindependent three-axis magnetometer calibration. Journal of Guidance Control and Dynamics, 28(1):115-120, 2005.

[11] Jon Crowell. Small AUV for hydrographic applications. In OCEANS 2006, pages 1-6. IEEE, 2006. 
[12] Mattia De Agostino, Ambrogio Maria Manzino, and Marco Piras. Performances comparison of different MEMS-based IMUs. In 2010 IEEE/ION Position Location and Navigation Symposium (PLANS), pages 187-201. IEEE, 2010.

[13] Maria de Bento, B Eissfeller, and F Machado. How to deal with low performance IMUs in an integrated navigation system: Step by step. In 2010 5th ESA Workshop on Satellite Navigation Technologies and European Workshop on GNSS Signals and Signal Processing (NAVITEC), pages 1-10. IEEE, 2010.

[14] Fredrik Dukan and Asgeir J Sørensen. Integration filter for APS, DVL, IMU and pressure gauge for underwater vehicles. IFAC Proceedings Volumes, 46(33):280-285, 2013.

[15] Mohammed El-Diasty and Spiros Pagiatakis. Calibration and stochastic modelling of inertial navigation sensor errors. Journal of Global Positioning Systems, 7(2):170-182, 2008 .

[16] Ryan M Eustice, Hanumant Singh, and Louis L Whitcomb. Synchronous-clock, oneway-travel-time acoustic navigation for underwater vehicles. Journal of Field Robotics, 28(1):121-136, 2011.

[17] Ryan M Eustice, Louis L Whitcomb, Hanumant Singh, and Matthew Grund. Recent advances in synchronous-clock one-way-travel-time acoustic navigation. In OCEANS 2006, pages 1-6. IEEE, 2006.

[18] Ryan M Eustice, Louis L Whitcomb, Hanumant Singh, and Matthew Grund. Experimental results in synchronous-clock one-way-travel-time acoustic navigation for autonomous underwater vehicles. In Proceedings 2007 IEEE International Conference on Robotics and Automation, pages 4257-4264. IEEE, 2007.

[19] Erin Fischell, Toby Schneider, and Henrik Schmidt. Design, implementation, and characterization of precision timing for bistatic acoustic data acquisition. IEEE Journal of Oceanic Engineering, 41(3):583-591, 2016.

[20] W Flenniken, J Wall, and D Bevly. Characterization of various IMU error sources and the effect on navigation performance. In ION GNSS, pages 967-978, 2005.

[21] Lee E Freitag, Matthew Grund, Jim Partan, Keenan Ball, Sandipa Singh, and Peter Koski. Multi-band acoustic modem for the communications and navigation aid AUV. In OCEANS, 2005. Proceedings of MTS/IEEE, pages 1080-1085. IEEE, 2005.

[22] Aditya S Gadre and Daniel J Stilwell. Toward underwater navigation based on range measurements from a single location. In Proceedings 2004 IEEE International Conference on Robotics and Automation, ICRA'04., volume 5, pages 4472-4477. IEEE, 2004.

[23] Aditya S Gadre and Daniel J Stilwell. A complete solution to underwater navigation in the presence of unknown currents based on range measurements from a single location. In 2005 IEEE/RSJ International Conference on Intelligent Robots and Systems, pages 1420-1425. IEEE, 2005. 
[24] Eric Gallimore, Jim Partan, Ian Vaughn, Sandipa Singh, Jon Shusta, and Lee Freitag. The WHOI Micromodem-2: A scalable system for acoustic communications and networking. In OCEANS 2010, pages 1-7. IEEE, 2010.

[25] Alan T Gardner and John A Collins. Advancements in high-performance timing for long term underwater experiments: A comparison of chip scale atomic clocks to traditional microprocessor-compensated crystal oscillators. In OCEANS, 2012, pages 1-8. IEEE, 2012.

[26] Alan T Gardner and John A Collins. A second look at chip scale atomic clocks for long term precision timing. In 2016 MTS/IEEE OCEANS Monterey, pages 1-9. IEEE, 2016.

[27] Pengfei Gui, Liqiong Tang, and Subhas Mukhopadhyay. MEMS based IMU for tilting measurement: Comparison of complementary and Kalman filter based data fusion. In 2015 IEEE 10th Conference on Industrial Electronics and Applications (ICIEA),, pages 2004-2009. IEEE, 2015.

[28] Albert S Huang, Edwin Olson, and David C Moore. LCM: Lightweight communications and marshalling. In Intelligent robots and systems (IROS), 2010 IEEE/RSJ international conference on, pages 4057-4062. IEEE, 2010.

[29] Mary M Hunt, William M Marquet, Donald A Moller, Kenneth R Peal, Woollcott K Smith, and Robert C Spindel. An acoustic navigation system. Technical report, Woods Hole Oceanographic Institution, 1974.

[30] Michael V Jakuba, James C Kinsey, James W Partan, and Sarah E Webster. Feasibility of low-power one-way travel-time inverted ultra-short baseline navigation. In OCEANS'15 MTS/IEEE Washington, pages 1-10. IEEE, 2015.

[31] Rudolph Emil Kalman. A new approach to linear filtering and prediction problems. Transactions of the ASME-Journal of Basic Engineering, 82(Series D):35-45, 1960.

[32] James H Kepper IV, Brian C Claus, and James C Kinsey. MEMS IMU and one-waytravel-time navigation for autonomous underwater vehicles. In MTS/IEEE OCEANS'17 Aberdeen. IEEE, 2017, Accepted, to appear.

[33] James C Kinsey, Ryan M Eustice, and Louis L Whitcomb. A survey of underwater vehicle navigation: Recent advances and new challenges. In International Federation of Automatic Control (IFAC) Conference of Maneuvering and Control of Marine Craft, volume 88, 2006.

[34] Nak Yong Ko, Hyun Taek Choi, Chong-Moo Lee, and Yong Seon Moon. Attitude estimation using depth measurement and AHRS data for underwater vehicle navigation. In OCEANS 2016 Shanghai, pages 1-4. IEEE, 2016.

[35] In-Uk Lee, Hang Li, Nhat-Minh Hoang, and Jang-Myung Lee. Navigation system development of the underwater vehicles using the GPS/INS sensor fusion. In 2014 14th International Conference on Control, Automation and Systems (ICCAS), pages 610-612. IEEE, 2014. 
[36] D. K. Maczka, A. S. Gadre, and D. J. Stilwell. Implementation of a cooperative navigation algorithm on a platoon of autonomous underwater vehicles. In OCEANS 200\%, pages $1-6$, Sept 2007.

[37] Leonard A McGee and Stanley F Schmidt. Discovery of the Kalman filter as a practical tool for aerospace and industry. 1985.

[38] Liam Paull, Sajad Saeedi, Mae Seto, and Howard Li. AUV navigation and localization: A review. IEEE Journal of Oceanic Engineering, 39(1):131-149, 2014.

[39] J-P Peyronnet, R Person, and F Rybicki. Posidonia 6000: a new long range highly accurate ultra short base line positioning system. In OCEANS'98 Conference Proceedings, volume 3, pages 1721-1727. IEEE, 1998.

[40] Nicholas R Rypkema, Erin M Fischell, and Henrik Schmidt. One-way travel-time inverted ultra-short baseline localization for low-cost autonomous underwater vehicles. In International Conference on Robotics and Automation (ICRA) 2017, pages 4920-4926. IEEE, 2017.

[41] Mohammad Shabani Sheijani, Asghar Gholami, Narjes Davari, and Mehdi Emami. Implementation and performance comparison of indirect Kalman filtering approaches for AUV integrated navigation system using low cost IMU. In 2013 21st Iranian Conference on Electrical Engineering (ICEE), pages 1-6. IEEE, 2013.

[42] Taek Lyul Song. Observability of target tracking with range-only measurements. IEEE Journal of Oceanic Engineering, 24(3):383-387, 1999.

[43] Alessandro Tagliabue, Laurent Bopp, Jean-Claude Dutay, Andrew R Bowie, Fanny Chever, Philippe Jean-Baptiste, Eva Bucciarelli, Delphine Lannuzel, Tomas Remenyi, Geraldine Sarthou, et al. Hydrothermal contribution to the oceanic dissolved iron inventory. Nature Geoscience, 3(4):252, 2010.

[44] Andrew F. Thompson, Yi Chao, Steve Chien, James Kinsey, M. Mar Flexas, Zachary K. Erickson, John Farrara, David Fratantoni, Andrew Branch, Selina Chu, Martina Troesch, Brian Claus, and James Kepper. Satellites to seafloor: Toward fully autonomous ocean sampling. Oceanography, Accepted, to appear.

[45] David Titterton and John L Weston. Strapdown Inertial Navigation Technology. Peter Peregrinus Ltd, 1997.

[46] Giancarlo Troni and Louis L Whitcomb. Adaptive estimation of measurement bias in three-dimensional field sensors with angular rate sensors: Theory and comparative experimental evaluation. In Robotics: Science and Systems, 2013.

[47] Derya Unsal and Kerim Demirbas. Estimation of deterministic and stochastic IMU error parameters. In 2012 IEEE/ION Position Location and Navigation Symposium (PLANS), pages 862-868. IEEE, 2012.

[48] Oscar A Viquez, Erin M Fischell, Nicholas R Rypkema, and Henrik Schmidt. Design of a general autonomy payload for low-cost AUV R\&D. In 2016 IEEE/OES Autonomous Underwater Vehicles (AUV), pages 151-155. IEEE, 2016. 
[49] Jeffrey M Walls, Stephen M Chaves, Enric Galceran, and Ryan M Eustice. Belief space planning for underwater cooperative localization. In 2015 IEEE/RSJ International Conference on Intelligent Robots and Systems (IROS), pages 2264-2271. IEEE, 2015.

[50] Jeffrey M Walls, Alexander G Cunningham, and Ryan M Eustice. Cooperative localization by factor composition over a faulty low-bandwidth communication channel. In 2015 IEEE International Conference on Robotics and Automation (ICRA), pages 401-408. IEEE, 2015.

[51] Jeffrey M Walls and Ryan M Eustice. Experimental comparison of synchronous-clock cooperative acoustic navigation algorithms. In OCEANS'11 MTS/IEEE KONA, pages 1-7. IEEE, 2011.

[52] Sarah E Webster. Decentralized Single-Beacon Acoustic Navigation: Combined Communication and Navigation for Underwater Vehicles. PhD thesis, 2010.

[53] Sarah E Webster, Ryan M Eustice, Christopher Murphy, Hanumant Singh, and Louis L Whitcomb. Toward a platform-independent acoustic communications and navigation system for underwater vehicles. In OCEANS 2009, pages 1-7. IEEE, 2009.

[54] Sarah E Webster, Ryan M Eustice, Hanumant Singh, and Louis L Whitcomb. Advances in single-beacon one-way-travel-time acoustic navigation for underwater vehicles. The International Journal of Robotics Research, 31(8):935-950, 2012.

[55] Sarah E Webster, Lee E Freitag, Craig M Lee, and Jason I Gobat. Towards realtime under-ice acoustic navigation at mesoscale ranges. In 2015 IEEE International Conference on Robotics and Automation (ICRA), pages 537-544. IEEE, 2015.

[56] Sarah E Webster, Louis L Whitcomb, and RM Eustice. Preliminary results in decentralized estimation for single-beacon acoustic underwater navigation. Robotics: Science and Systems VI, pages 1-8, 2010.

[57] Eric Wolbrecht, Michael Anderson, John Canning, Dean Edwards, Jim Frenzel, Doug Odell, Tom Bean, Jordan Stringfield, J. Feusi, B. Armstrong, A. Folk, and B. Crosbie. Field testing of moving short-baseline navigation for autonomous underwater vehicles using synchronized acoustic messaging. Journal of Field Robotics, 30(4):519-535, 2013. 